\title{
BENDIX KANSAS CITY DIVISION \\ TECHNOLOGICAL SPINOFF \\ THROUGH 1978
}

BDX-613-2141

Internal Distribution February 1979

Prepared by:

H. T. Barnes

Department 554

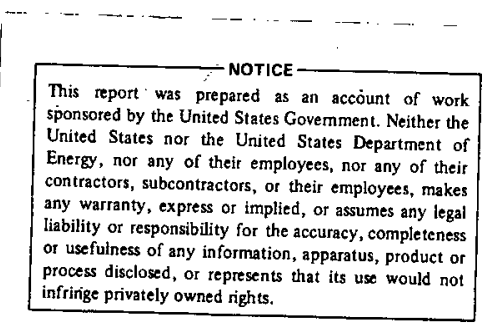

Communications Services

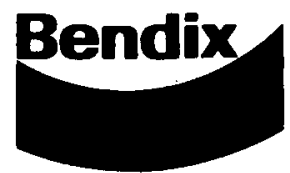

Kansas City Division 


\section{DISCLAIMER}

This report was prepared as an account of work sponsored by an agency of the United States Government. Neither the United States Government nor any agency Thereof, nor any of their employees, makes any warranty, express or implied, or assumes any legal liability or responsibility for the accuracy, completeness, or usefulness of any information, apparatus, product, or process disclosed, or represents that its use would not infringe privately owned rights. Reference herein to any specific commercial product, process, or service by trade name, trademark, manufacturer, or otherwise does not necessarily constitute or imply its endorsement, recommendation, or favoring by the United States Government or any agency thereof. The views and opinions of authors expressed herein do not necessarily state or reflect those of the United States Government or any agency thereof. 


\section{DISCLAIMER}

Portions of this document may be illegible in electronic image products. Images are produced from the best available original document. 

This document replaces BDX-613-1984, Bendix Kansas City Division $X$
Technological Spinoff Through 1977. That report can now be discarded.

WAM-myw

This report was prepared as an account of work sponsored by the United States Government. Neither the United States, nor the United States Department of Energy, nor any of their employees, nor any of their contractors, subcontractors, or their employees, makes any warranty, expressed or implied or assumes any legal liability or responsibility for the accuracy, completeness or usefulness of any information, apparatus, product, or process disclosed. or represents that its use would not infringe privately owned rights.
The Bendix Corporation

Kansas City Division

P. O. Box 1159

Kansas City, Missouri 64141

A prime contractor with the United States Department of Energy under Contract Number DE-AC04-76-DP00613 
CONTENTS

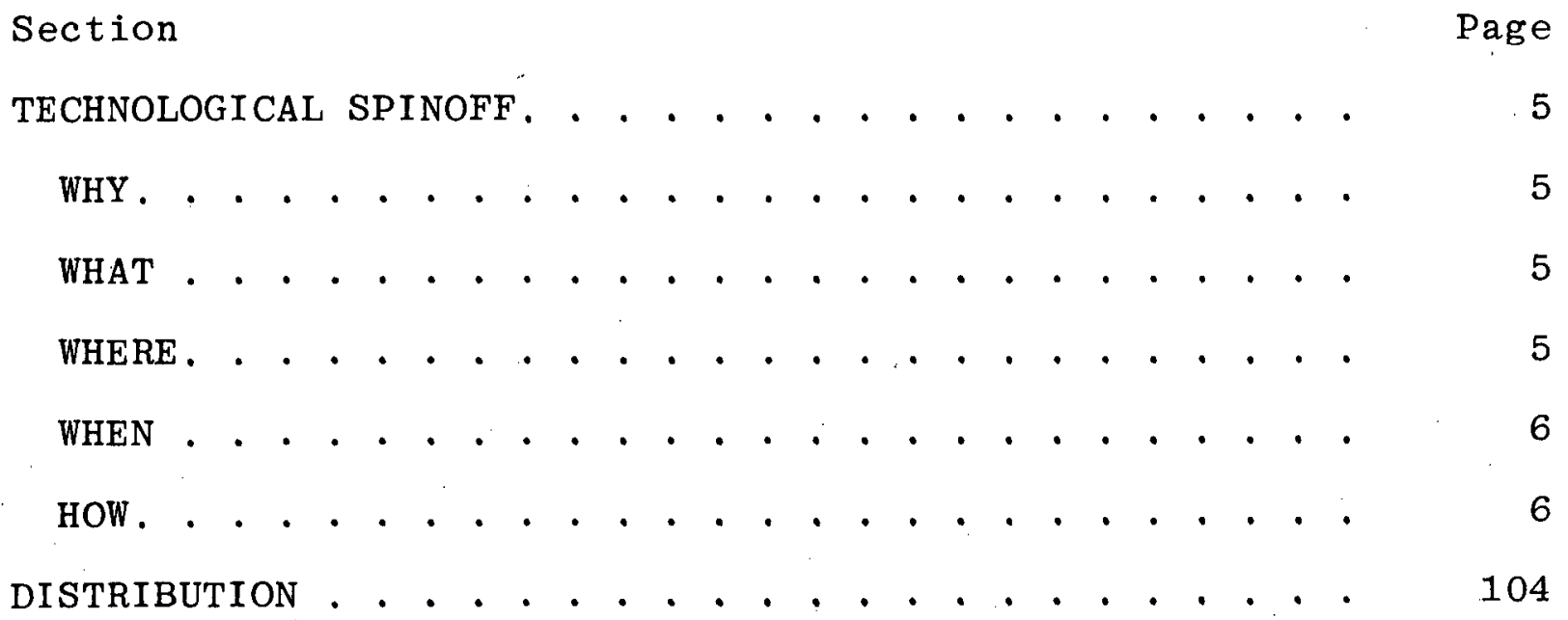




\section{APPENDICES}

Page

A. REPORT COLLECTIONS IN THE UNITED STATES. . . . . . $\quad 7$

B. REPORT COLLECTIONS IN OTHER COUNTRIES . . . . . . . 11

C. REPORT COLLECTIONS IN INTERNATIONAL AGENCIES . . . 14

D. TECHNOLOGICAL SPINOFF DOCUMENTS RELEASED . . . . . 16

E. AUTHOR INDEX OF REPORTS RELEASED . . . . . . . . . 49

F. SUBJECT INDEX OF REPORTS RELEASED. • . . . . . . . 77

G. DRAWING SETS RELEASED. . . . . . . . . . . . . 102 


\section{TECHNOLOGICAL SPINOFF}

WHY

One important objective of the Department of Energy's (DOE)

technical information program is "to encourage, wherever feasible, the preparation of information resulting from research and development activities in a form suitable for unlimited distribution."l Essentially, since the work is paid for by tax money, the results should be made available to the taxpayer. Thus, release of such information is intended to enhance technological progress throughout the scientific and industrial communities.

WHAT

Most technological spinoff material produced by Bendix Kansas City Division is in the form of unclassified technical reports. These reports include regular reports published in accordance with reporting requirements and reports prepared especially for technological spinoff.

Classified reports can be spun off also, but only if they are not Atomic Weapon Data. Other types of technological spinoff are conference papers, journal articles, and books. Some sets of drawings of unique Bendix-designed equipment have been released.

\section{WHERE}

When the necessary approvals have been obtained, spinoff documents are sent to DOE's Technical Information Center in Oak Ridge. The Technical Information Center (TIC) makes a standard distribution in microfiche form of the document to various subscribing government agencies and contractors, and announces the document in Energy Research Abstracts (ERA). The documents also are placed in technical libraries around the United States (Appendix A) and in many international information collections (Appendices $\dot{B}$ and $C$ ).

After distribution, the documents are available for purchase in the United states from the National Technical Information Service (U.S. Department of Commerce) in Springfield, Virginia, and from a company which contracts with DOE to sell microform copies of these documents.

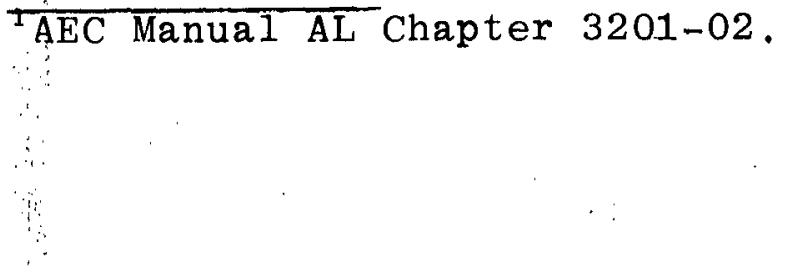


WHEN

Spinoff documents are forwarded to TIC as soon as possible after internal distribution. After the documents are sent to TIC, a period of 3 to 4 months usually elapses before announcement in NSA and availability of the documents from NTIS. : Conference papers are held for release by TIC until the conference is over.

HOW

After a spinoff candidate document is distributed internally, the additional approvals required for public release of the document are obtained. For a technical report, these approvals are Security and Legal (patent) approvals. These approvals are obtained by the technological spinoff coordinator in the Communications Services department.

After the approvals have been obtained, the required number of copies with the proper documentation are forwarded to DOE's Technical Information Center. From there, the reports are announced and made available worldwide.

Many of the reports released have required slight changes before they became eligible for unrestricted distribution. Consequently, the spinoff report may be different from the report distributed within the weapons complex. Because of this, and for reasons of economy, most requestors of Bendix reports are advised that the reports are available for purchase from NTIS. Requestors from outside the United States are also advised of the location of the nearest international information collection where the report may be available. 
Appendix A

REPORT COLLECTIONS IN

THE UNITED STATES 


\title{
Appendix A
}

REPORT COLLECTIONS IN THE UNITED STATES

\author{
ALABAMA \\ Auburn, Auburn University \\ Tuskegee Institute, Tuskegee Institute \\ ARIZONA \\ Tucson, University of Arizona \\ CALIFORNIA \\ Davis, University of California \\ Los Angeles, University of California \\ Santa Barbara, University of California \\ COLORADO \\ Boulder, University of Colorado \\ DISTRICT OF COLUMBIA \\ Washington, Library of Congress \\ FLOR IDA \\ Gainesville, University of Florida \\ GEORGIA \\ Atlanta, Georgia Institute of Technology \\ HAWAI I \\ Honolulu, University of Hawaii \\ IDAHO \\ Pocatello, Idaho State University \\ ILLINOIS \\ Urbana, University of Illinois \\ INDIANA \\ Lafayette, Purdue University \\ IOWA \\ Ames, Iowa State University. \\ KANSAS \\ Manhattan, Kansas State University \\ KENTUCKY \\ Lexington, University of Kentucky
}


MARYLAND

Baltimore, Johns Hopkins University

College Park, University of Maryland

MASSACHUSETTS

Cambridge, Massachusetts Institute of Technology

Worcester, Worcester Polytechnic Institute

MICHIGAN

Ann Arbor, University of Michigan

MISSISSIPPI

State College, Mississippi State University

MISSOURI

Columbia, University of Missouri

Kansas City, Linda Hall Library

NEW JERSEY

Princeton, Princeton University

NEW MEXICO

Albuquerque, University of New Mexico

NEW YORK

Albany, New York State Library

Albany, State University at Albany

Ithaca, Cornell University

New York, Columbia University

Rochester, University of Rochester

Syracuse, Syracuse University

NORTH CAROLINA

Raleigh, North Carolina State University

OHIO

Cincinnati, University of Cincinnati

Cleveland, Cleveland Public Library

Toledo, University of Toledo

\section{OKLAHOMA}

Norman, University of Oklahoma

\section{PENNSYLVANIA}

Philadelphia, University of Pennsylvania

Pittsburgh, Carnegie Library

University Park, Pennsylvania State University

PUERTO RICO

San Juan, University of Puerto Rico 
SOUTH CAROLINA

Columbia, University of South Carolina

TENNESSEE

Knoxville, University of Tennessee

TEXAS

Austin, University of Texas

College Station, Texas A \& M University

Houston, Rice University

UTAH

Salt Lake City, University of Utah

VIRGINIA

Blacksburg, Virginia Polytechnic Institute Charlottesville, University of Virginia

WASHINGTON

Pullman, Washington State University

Seattle, University of Washington

WEST VIRGINIA

Morgantown, West Virginia University

WISCONSIN

Madison, University of Wisconsin 
Appendix B

REPORT COLLECTIONS

IN OTHER COUNTRIES 


\author{
Appendix B \\ REPORT COLLECTIONS IN OTHER COUNTRIES
}

\title{
ARGENTINA
}

Buenos Aires, Comisión Nacional de Energía Atomica

\section{AUSTRALIA}

Canberra, National Library of Australia

Sutherland, New South Wales, Atomic Energy Research Establishment

AUSTRIA

Vienna, Zentralbibliothek der Physikalischen Institute der Universität

BELGIUM

Mol-Donk, Centre d'Etude de l'Energie Nucléaire

BRAZIL

Rio de Janeiro, Comissao Nacional de Energia Nuclear

Sao Paulo, Instituto de Energia Atómica

\section{CANADA}

Chalk River, Ontario, Atomic Energy of Canada, Ltd., Chalk River

Hamilton, Ontario, McMaster University

Ottawa, National Research Council

CONGO, REPUBLIC OF

Kinshasa, Trico Nuclear Center

DENMARK

Risö, Danish Atomic Energy Commission

FINLAND

Otaniemi, Teknillinen Korkeakoulu

FRANCE

Saclay, Gif-sur-Yvette, Centre d'Etudes Nucléaires de Saclay

GERMANY, WEST

Karlsruhe, Zentralstelle für Atomkernenergie Dokumentation

Munich, Technische Universität

GREECE

Athens, Nuclear Research Center, Democritus Library

INDIA

Bombay, Trombay, Bhabha Atomic Research Center Library 
IRAQ, REPUBLIC OF

Baghdad, Atomic Energy Commission, Nuclear Research Institute

IRELAND

Dublin, University College

ISRAEL

Yavne, Israel Atomic Energy Commission, Soreq Nuclear Research Centre

ITALY

Rome, Centro di Studi Nucleari della Casaccia

JAPAN

Tokyo, National Diet Library

Tokai-Mura, Naka-gun, Ibaraki-ken, Japan Atomic Energy

Research Institute

KOREA, REPUBLIC OF

Seoul, Atomic Energy Research Institute

MEXICO

Mexico, D. F., Comisión Nacional de Energía Nuclear

NETHERLANDS, THE

Petten, Reactor Centrum Nederland

The Hague, Bibliothek Octrooiraad

NORWAY

Kjeller, Institutt for Atomenergi

PORTUGAL

Sacavem, Junta de Energia Nuclear

SOUTH AFRICA, REPUBLIC OF

Pretoria, Atomic Energy Board Library

SPAIN

Madrid, Junta de Energia Nuclear, Ciudad Universitaria

SWEDEN

Nyköping, Aktiebolaget Atomenergị Library

SWITZERLAND

Zürich, Swiss Federal Institute of Technology

UNITED KINGDOM

Harwell, Didcot, Berkshire, Atomic Energy Research Establishment 
Appendix C

REPORT COLLECTIONS IN

INTERNATIONAL AGENCIES 
Appendix C

REPORT COLLECTIONS IN INTERNATIONAL AGENCIES

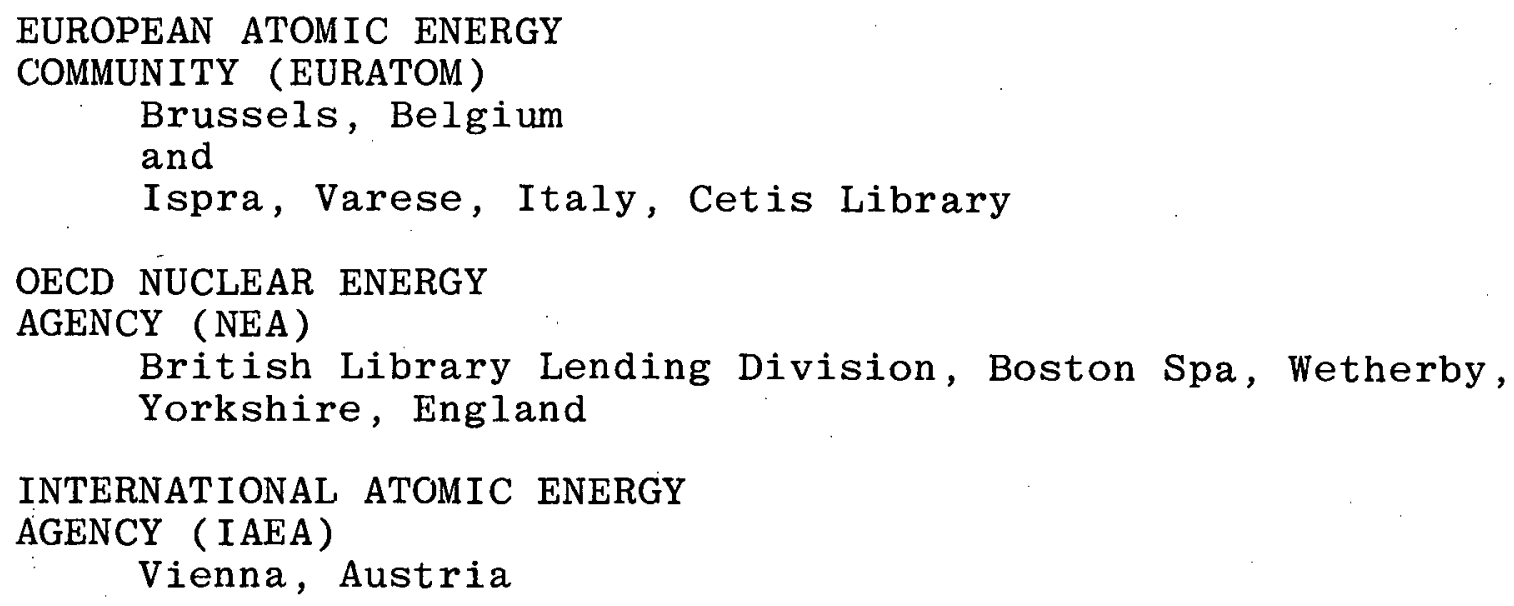


Appendix D

TECHNOLOGICAL SPINOFF

DOCUMENTS RELEASED 
TECHNOLOGICAL SPINOFF DOCUNENTS RELEASED

DOCUMENT

NUMBER

BDX-613- TITLE

1

2

APPLICATION OF PUNCHED CARO TECHNIQUES FOA OPTIMLZING RELIABILITY

DATE OF AUTHOR (S) - J.J.HOEFER, J.H.SWAFFORD

$02 / 15 / 59$

PRODUCTION OF A RELAY UNOER CONTROLLED CONOITIONS

$03 / 15 / 60$ AUTHOR (S) - K.U.NEMTON

$06 / 60$ AUTHOR(S) - S.J.CATALANS

A THEORETICAL TREATAENT OF LOW VOLTAGE CONTINUOUS SPECTRUM X-RADIATION AUTHOR(S) - J.S.CARSON, N.K.HEARN

THE TARNISH RESISTANCE OF GOLO PLATING DVER SILVER AUTHOR(S) - H.B.HARDING

ADVANTAGES OF GLASS MICRO-BALLOONS AS FILLER MATERIAL AUTHOR (S) - F.R. HALTERBACH

CONOUCTIVE ADHESIVES-A SUBSTITUTE FOR SOLOER AUTHOR (SI - R.L.MARTIN

a VARIABLE PHASE, CONSTANT USWR, ANTENNA LOAD AUTHORIS) - T.J.RUSSELL

SEMICONDUCTOR FAILURE ANALYSIS USIME THE CHARACTERISTIC CURVE AUTHOR (S) - R.A.HOLLADAY

AUDARE-A DEVICE FOR RECOROING DATA AUTHOR (S) - M.R.EASTERDAY

$06 / 17 / 60$

$06 / 14 / 60$

$08 / 10 / 60$

$08 / 10 / 60$

$09 / 02 / 60$

$09 / 29 / 60$

$10 / 60$

EFFECTS OF TEMPERATURE ON FILLED EPOXY ENCAPSULATION MATERIALS

$10 / 14 / 60$ AUTHOR(S) - B.A.OAVIS

SURFACE PREPARATION OF PLASTICS FOR ADHESIYYE BONDING

$11 / 02 / 60$ AUTHOR (S) - L.C.JACKSON

A TRANSISTORIZED HIGH INPUT IMPEOANCE AMPLIFIER

$12 / 60$ AUTHORISI - D.M.REED

ULTRASONIC SPOT MELD EVALUATION

$03 / 61$ AUTHOR (S) - D.H.DRAYER

USES OF LOW VOLTAGE X-RAY TUBES WITH THIN BERYLLIUM MINDOWS

$03 / 61$ AUTHOR (S) - H. CARTER

EFFECT OF DIISOCYANATE STRUCTURE ON LOAO-BEARING PROPERTIES OF FLEXIBLE URETHANE FOAMS

AUTHOR (S) - C.A.PETERSON, C.H.SMITH

TOTAL PURChASING DATA PROCESSING SYSTEM AUTHOR(S) - J. S. JACKSON

CONOUCTIVITY TESTING OR EDOY CURRENT TESTING WITH THE FM-IOOMAGNATEST CONDUCTIVITY METER AUTHOR(S) - W.A.GANQUIST

\section{AN ANALDG COMPUTER FOR DEVELOPMENT PROJECT SCHEbUL ING}

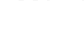

$03 / 07 / 61$

$04 / 21 / 61$ AUTHOR $(S I=$ D.H.HAX

\section{CAST FLEXIBLE URETHANE POLYMERS} AUTHOR (S) - C.A.PETERSON, C.H.SMITH

$05 / 61$

DEgRADATION OF GLASS VACUUM TUBES AS a RESULT OF hel IUM PERMEATION AUTHORISI - J.J.RODE

A METHOD OF EXPOSURE CONTROL FOR ELECTRON MICROSCOPY

$05 / 61$

$05 / 61$

$07 / 61$

$07 / 10 / 61$

$08 / 61$ AUTHOR(S) - G.F.OSBORNE

$08 / 61$

POTTING PROBLEMS RELATE
AUTHOR(S) - B.A.DAVIS

$09 / 20 / 61$ AUTHORISI - W.B.HARDING

$10 / 61$ AUTHOR (S) - J.E.AUSTIN, L.C.JACKSON 

AUTHOR (S) - T.J.RUSSELL, V.E.SCHUSTER AUTHORIS) - F.A.SPIES

PHYSICAL PROPERTIES OF ELECTROFORMED NICKEL FROM SULFAMATE SOLUT IONS AUTHORISI - R.K.ASHER, N.B.HAROING

TOTAL PRDCUREAENT SYSTEM PLAN AUTHOR(S) - J.S.JACKSON

TIMESAVING Hith a PARALlel AMALYiIng CHAMNEL atTACHAENT AUTHOR(S) - J.S.CARSON

GENERAL TECHNOLOGY OF ADHES IVES AUTHOR(S) - L.C.JACKSON

\section{AUTOMATIC MEASURING OF TEMPERATURE COEFFICIENTS} AUTHOR(S) - L.A.SCHLESSELMAN, E.M.SIEGEEN

$10 / 19 / 61$

$10 / 26 / 61$

$11 / 61$

$04 / 29162$

$05 / 62$

$05 / 62$

$05 / 10 / 62$

$05 / 31 / 62$

$06 / 11 / 62$

$09 / 13 / 62$

$11 / 62$

THE ANALYSIS OF TIN-IRIDIUM-ZINC ALLOYS

$12 / 21 / 62$ AUTHORIS) - M.V.DAVIS, JR:

$03 / 63$

RIGID URETHANE FOAMS FROM E-CAPROLACTONE POLYESTERS

04106163

MODERN GAGE CONTROL

$04 / 06 / 63$ AUTHORISI - L.F.TSCHECHTELIN

$05 / 27 / 63$

SOLDERING TO GQLD PLATING

$07 / 63$ AUTHORIS) - C.H.SHITH

BONDING FLUOROHAL OCARBONS TO COPPER FOR ELECTRICAL APPLICATIONS

$11 / 63$ AUTHORISI - L.C.JACKSON

$08 / 26 / 63$ A STANOAROS AND CAL IBRATI
AUTHDRISI - J.F.HADLEY

MANAGEMENT CONSIDERATIONS FOR CLEAN MORKING AREAS

$09 / 63$ AUTHOR(S) - R.R.GRINER

$09 / 63$ LEAD ZIRCONATE-LEAD TITANATE FERROELECTRICS AUTHORISI - R.H.OUUNGAN, T.F.MURRATY AUTHOR(S) - G.F.OSBORNE

$09 / 63$

BINOMIAL SAMPLING PLANS INDEXED BY AQL ANO LTPO AUTHOR(S) - R.L.KIRKPATRICK

$09 / 30 / 63$ AUTHORISI - C.J.HIEGANO

$10 / 63$ MEAN RANGE AUTHOR(S) - R.L.KIRKPATRICK

WRITING METAL FINISHING PROCCESS SPECIFICATIONS

$10 / 03 / 63$ AUTHORISI - M.B.HARDING

THE DETERMINATION OF THE ELECTROMOTIVE FORCE OF A SATURATED STANDARD CELL AUTHOR(S) - G.H.RHODES

$11 / 12 / 63$ 
THERMOPLASTIC FRONTIER LAMS-GOOD AMO BAO GUYS AUTHOR $(S)$ - J.C.DALY

GETTING MORE DUT OF PLATING THRUGH PROCESS SPECIFICATIONS

$12 / 63$

$03 / 64$

$03 / 64$

$04 / 22 / 64$

$03 / 22 / 64$

$03 / 24 / 64$

$05 / 64$

$05 / 18 / 64$ AUTHOR(S) - H.B. HARDING

PRODUCTION TESTING DF MAGNETIC CORE SHIFT REGISTER ELEMENTS

$07 / 01 / 64$

$07 / 15 / 64$ AUTHOR(S) - K.D.BENEOICT

RESISTANCE MEASUREMENTS BY RATIO EMPLOYIMG VOLTAGE TO FREOUENCY CONVERTERS

$08 / 64$ AUTHOR(S) - K.H.BRACKNEY

THE ANALYSIS OF AN INPUT BUFFER FOR REMOTE CONTROL OF LOGIC CIRCUITS AUTHOR(S) - H.G.THORPE

$11 / 64$

A CAST FLEXIBLE ESTER-URETHANE POLYMER

$01 / 02 / 64$ AUTHOR (S) - C.H.SHITH

EL ECTRDOEPOSITION OF NICKEL-COBALT ALLOY: OPERATING PARAMETERS AND PHYSICAL PROPERTIES DF THE DEPOSITS AUTHOR(S) - D.H.ENDICOTT, J.R.KNAPP

a single pulse peak reading voltmeter AUTHORISI - A.F.SCHMIDT

EFFECTS OF SURFACE PREPARATION ON BONO STRENGTHS OF MAGNESIUH AUTHOR (S) - L.C.JACKSON

THERMAL CHARACTERISTICS OF SOME RIGID URETHANE FOAMS AUTHOR (S) - C.H.SMITH

$09 / 64$

$02 / 65$

$03 / 10 / 65$

A NEW DEYELOPMENT IN POL YURETHANE ELASTOMER PROCESSING HHICH REDUCES CRACK SUSCEPTIBILITY ANO BUBBLE ENTRAPMENT AUTHORISI - D.0.0SWALO

A SLOW TIME BASE REAODUT SYSTEH FOR NON-REPETITIVE PULSE ANALYSIS AUTHORISI - C.E.THOMSON

$04 / 07 / 65$

$04 / 15 / 65$ A SENSITIVE, hIGH-SPEED, DC VOLTAGe Comparator AUTHOR(S) - R.G.PIPER

SOLDERABILITY TESTING AUTHOR SI - W.B.HARDING

POLYURETHANE CABLE FABRICATION FOR SMALL QUANTITIES

02165 AUTHORISI - H.P.MCKAY

$09 / 15 / 65$ EFFECT OF MOLECULAR STRUCTURE ON THE AGING PROPERTIES OF FLEXIBLE URETHANE FOUAS

TEST EQUIPMENT ACCURACY AUTHORIS) - G.D.WINKLER 

AUTHDR(S) - O.L.GIBSON, C.A.STEVENS

destgn of a variagle phase, constant VSHR, microwaVe load

$05 / 65$

CONFIDENCE LIMITS ON A PROPORTION DEFECTIVE CMARACTERIzEO BY THO

$07 / 65$ SPECIFICATION LIMITS

AUTHORISI - R.L.KIRKPATRICK

A METHOD FOR INTERRUPTING A HIGH STRAIN RATE TENSION TEST BEFORE SPECIMEN FRACTURE

AUTHOR(S) - C.B.BARNES, M.V.OAVIS, JR.. E.L.MAMKINS

GRAPHIC AIO TO DESIGN OF SPLICED QUARTER-MAVE RESONANT LINES AUTHORISI - J.R.LEMBKE

RADIUS GENERATION IN VIBRATORY DEBURRING AUTHOR(S) - G.V.ROBBINS COMPARATIVE DUCTILITY OF BRITTLE METALS OY A PRECISION ELONGATIDN TECHNIQUE AUTHORIS) - E.P.PATTERSON

THE EFFECT OF HIGH-STRAIN-RATE FORMING ON THE RECRYSTALIZATION OF COPPER AUTHOR(S) - D.L.GIBSON, C.A.STEVENS AUTHORIS) - V.L.MILLERTON

THE PROPERTIES OF ALUMINUH ANO COPPER AFTER HIGH-ENERGY-RATE FORMING AUTHOR(S) - D.L.GIBSON, C.A.STEVENS 
104 FEASIBILITY STUDY: PLATING THICKMESS GAGE AUTHORISI - F.J.QARKER

105 BORON TRIFLUORIDE-AMINE ADOUCTS AS HARDENERS FOR LIOUIO EPOXY RESINS AUTHORISI - C.H.SMITH

107 NONDESTRUCTIVE TESTING OF PERCUSSIVE ARC MELDS NONDESTRUCTIVE TESTIMG OF PERCUSSIVE ARC NELDS
AUTHORISI - C.C ESTEP. D.S. GENSER, H.J.STONE

$08 / 68$

12168
SOLID LUBRICANT APPLICATION BY SPRAY AND TUMBLE METHODS AUTHORISI - F.A.CLAY
108 EPON BZB/MENTHANE DIAMINE CURING FACTORS AUTHOR (S) - C.J.CAMP
109
110 TENSILE TESTING OF HIGH STRENGTH MATER IALS AUTHOR(S) - F.H.LAMAR
111 SMALL ANCLE GENERATOR AUTHORTSI - R.J.RUSSELL
I12 CARBON FOAM-A CELLULAR STRUCTURAL MATERIAL FOR MIGH TEMPERATURE APPLICATION AUTHOR(S) - G.K.BAKER, R.P.CHARTOFF, A.K.THOENI JOINING HIGH-STRENGTH STEELS BY DIFFUSION BONOING AUTHOR(S) - A.M.BOSCH
114 COMPUTERIIEO EXOTHERM CALCULATIDNS FOR LARGE EPOXY CASTINGS AUTHOR (S) - D.D.SMITH
115 ELECTRODEPOSITION OF ALUAINUM AUTHORISI - F.A.CLAY, H.B.HAROING, C.J.STIMETZ
116 CHARACTERISTICS OF SKIP LOT SAMPLING
AUTHOR(S) - R.L.KIRKPATRICK
116 CHARACTERISTICS OF SKIP LOT SAMPLIME
AUTHOR(S) - R.L.KIRKPATRICK
1 i7 SOLUT IONS FOR THE HAGR SEQUENTIAL T-TEST, SEQUENTIAL VARIABLES SAMPLING TO CONTROL THE PERCENT DEFECTIVE AUTHOR (S) - R.L.KIRKPATRICK

$12 / 68$

$12 / 68$

1268

$12 / 68$

118 DESIGNING ADHES IVES AND ADHESIVE INTERFACES FOR OPTIMUM ELECTRICAL CONDUCT IVITY AUTHOR (S) - J.E.HESS, L.C.JACKSON TRANSISTOR CHARACTERIZATION AUTHOR(S) - J.R.LEMBKE AFTER OISSOLUTION IN 2-BUTANONE AUTHORISI - W.R.CARROLL AUTHOR(S) - F.N.LARSEN

$02 / 69$

02169

$04 / 69$

$06 / 69$

$07 / 69$

07169

07169

$08 / 69$

$09 / 69$

DESIGNING AND MOLOING MODULE HOUSINGS FOR ELECTRONIC PACKAGES AUTHOR(S) $=$ H.P. MCKAY TECHNIQUES IN USING POL YURETHANE MATER IALS FOR ENCAPSULATION AUTHORISI - W.P.MCKAY AUTHOR(S) - H.P.MCKAY AUTHOR(S) - E.R.CHERAMY

127 INTERFEROMETER PRISH: PDR 187 AUTHOR (S) - M.P.SCHOEPPNER

$02 / 70$

LEAD WIRE SOLDERABILITY TEST

02170 AUTHOR (S) - H.H.TURNER 

AUTHORISI - L. D. MONNELL

$06 / 69$

BONDING STRENGTH OF ENCAPSULATING MATERIALS TO MOOKUP WIRE

$06 / 70$

136 A DISTORTION ENERGY FAILURE THEORY FOR ORTHOTROPIC MATERIALS $\quad$ O6/70

136 A DISTORTION ENERGY FAILURE THEORY FOR ORTHOTROPIC MATERIALS $\quad$ O6/70

137 A STRESS ANALYSIS OF THICK-WALL ORTHOTROPIC SPHERICAL VESSELS

$06 / 70$ AUTHOR (S) - G.H.FORMAN

$07 / 70$

141 FLATPACK REFLOW SOL DERING AUTHORISI - D.H.BOWLEN, E.L.HOLLINGSWORTH

$08 / 70$

142 DESIGN OF JOINTS FOR FILAMENT-MOUNO CYLIMDERS

$11 / 16 / 70$

143 DESIGN ANO DEVELOPMENT OF THRE-OIMENSIOMAL INTERLOCKED,F ILAMENT-MOUND COMPOSITE AUTHORISI - G.H.FORMAN AUTHORISI - H.F.DARBYSHIRE

170 Effects of amine catalysts on the physical properties of a rigio urethane FOAM SYSTEM AUTHOR(S) - T.E.NEET 

REQUIREMENTS FOR REFLOW SOLOERING OF INTEGRATED CIRCUIT FLATPACKS

$07 / 28 / 71$

190 FILLED PAPER HONEYCOMB AUTHOR(S) - R.F.TAYLOR

$06 / 18 / 71$

194 BEAM LEAD SEMICONOUCTOR TESTING AND HANDLING AUTHORISI - L.W.SHITH

$12116 / 70$

195 RIGID EPOXY FOAM ENCAPSULATION

$07 / 19 / 74$

(REV.) AUTHOR(S) - F.C.AYERS

198 SPLIT FIBERGLASS HOLDER AUTHOR (S) - H.F.OARBYSHIRE

$01 / 14 / 71$

204 ELECTRICAL CONNECTION STUOY

$02 / 22 / 71$ AUTHOR(S) - R.D.WAGNER

205 maChINE COOLANT SELECTION and maIntenance manual

IREV.I AUTHORISI - V.A.LYONS

$08 / 27 / 71$

$10 / 13 / 70$

MEDIUM DENSITY MOLOED FOAM

AUTHOR(S) - J.R.FENDER

213 DESIGN OF AN INSULATOR WHICH MUST SURVIVE HIGH G-FDRCES AUTHORCSI - V.J.GULZO, L.F. THORNE

$01 / 14 / 71$

227 BORON SHOT FABR ICATION

(REV.) AUTHOR (S) - M.J.NAPIER

$12 / 21 / 71$

$10 / 08 / 70$ MEDIA-PLASTIC FOAM AUTHORISI - H.M.LEE, D.W.LIOU

233 STATE OF CURE OF PRINTED CIRCUIT BOARDS AUTHOR (S) - K.C.ATKINSON, J.W.STEELE

$02122 / 71$

234 WRITERS GUIDE TO THE PREPARATION OF ARTWORK FOR TECHNICAL REPORTS AUTHOR(S) - F.A.WALKER

$07 / 28 / 71$

$05 / 21 / 71$ AUTHORISI - R.A.HINES

236 ELECTROFORMEO GILDING METAL ROTATING BANO

0010171

ELECTROPOL ISHING CVO TUNGSTEN

$06 / 18 / 71$ AUTHORIS) - A.H.RAHE

$12 / 16 / 70$ AUTHOR(S) - J.R.FENDER

$07 / 23 / 72$

SYNTACTIC FOAH, FYTO-3 AUTHOR (S) - J.R.JOHNSTON

$02 / 19 / 72$ AUTHORIS) - C.U.FOTOPOULOS

$02 / 22 / 71$ AUTHOR (S) - M.F.MOYLE

$11 / 06 / 72$

THE EOI TOR'S NOTEBOOK AUTHORIS) - F.A.HALKER

$01 / 26 / 72$ AUTHOR (S) - F.A.WALKER

$02122 / 71$

AUTHORISI - H.F.MOYLE

269 CHEMICAL VAPOR DEPOSITION PROCESS EQUIPMENT

$06 / 07 / 71$ AUTHOR (S) - G.J.HALE

27.4 THE DETERMINATION OF AVERAGE CVCLE TIME FOR AN AUTOMATED WAREHOUSE AUTHORIS) - J.C.SMITH

$07 / 15 / 71$ COMPUTER AIDEO TEST SYSTEMS

$03 / 22 / 71$ AUTHORISI - J.A.HALTERS

276 OPTOELECTR ONIC MEASUREMENT

$03 / 22 / 71$ AUTHORISI - R.M.HOWARD 
291 INYESTMENT CASTING TO ENHANCE PROOUC IBILITYO-A CASE STUDY

$06 / 18 / 71$ AUTHOR(S) - M.E.CROMMELL

294 DESIGN EVALUATION OF INSULATOR PIM

$03 / 71$ AUTHOR(S) - R.D.LOHR

$04 / 71$ AUTHOR(S) - J.R.FEMDER

$05 / 11 / 72$

306+ CONFIRMATION OF A NAJET ORILLIMG TECHNIQUE FOR SMALL HOLES

$08 / 16 / 71$ AUTHOR(S) - J.M.HENRY

309 INSPECTION DF FINE-PITCHED GEARS

$02 / 22 / 71$ AUTHORISI - C.H.BERRY, JR.

$05 / 71$

315 FAST RECOVERY TIME DIODE TESTING

$03 / 71$ AUTHOR $(S)$ - R.R . FLOWER S

316 MECHANICAL PRODUCTS QUARTERLY

$03 / 22 / 71$ AUTHOR(S) - B.T.QUARTERLY

$07 / 28 / 71$

31

THE INCOMPLEAT STYLIST AUTHORIS) - F.A.WALKER

$03 / 71$ AUTHOR(S) - D.L.HOLLAR

$03 / 71$ AUTHOR(S) - D.L.HOLLAR AUTHOR(S) - M.V.DAVIS, JR.

$06 / 07 / 71$ AUTHOR(S) - D.M.BOMLEN

$06 / 07 / 71$

FLOU SOLDERING AND SUPPORTING PROCESSES

$06 / 07 / 71$ AUTHOR(S) - M.F.MOYLE

$03 / 22 / 71$ ASSEMBL IES ASSEMBL IES - F.H.LEWIS AUTHORI S) - M.C.RUTHERFORD

$07 / 11 / 72$

OIAGMOS IS OF MALFUACTIONS IN COMPLEX ELECTRONIC ASSEMBLIES

$06 / 18 / 71$

$03 / 71$

ELECTRONICS ENG INEER ING QUA
AUTHOR (SI - B.T.QUARTERLY 
349 EVALUATION OF METHOOS FOR COMPUTER-AIOED DESIEN OF TEST EQUIPMENT AUTHOR(S) - F. PAULIC III

353
(REV.

PROCESSING OF THIM CELLULAR SIL ICOME SLABS

DArE UF

$07 / 28 / 71$

(REV.) AUTHOR(S) - P.H.ALDRICH

355 MEASUREMENT METHOD FOR POLYSTYRENE

AUTHOR(SI - T. MATZKANIN

360

(REV.)

STRETCH EFFECTS ON LOAD DEFLECTION AND THICKMESS OF Y-3333 CELLULAR SILICONE

SLABS

AUTHOR (S) - P.H.ALORICH

363 THICK-FILM NETWORKS

IREV.) AUTHORISI - G.A.SHARTL

366 FINE-EDGE BLANKING AND PIERCING AUTHORISI - H.J.SEESE

369 THEORY AND OPERATION OF THE BENDIX MOLO HEATING ANO COOLING EQUIPMENT AUTHOR(S) - R.H.FLOERSCH

374 COMPRESSION PLATENS FOR LOAD VERSUS DEFLECTION TESTING OF SILICONE ELASTONERS

AUTHOR(S) - G.H.EDMAN

375

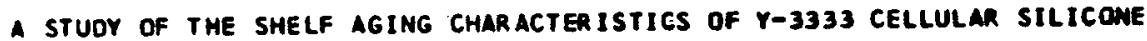

$08 / 20 / 71$

$06 / 07 / 71$

$09 / 07 / 71$

$05 / 11 / 72$

376 DNC COMMITTEE FINAL REPORT

AUTHORISI - D.L.SETTER

380 A STUOY OF THE OIMENSIONAL STABILITY OF MOLDED MOLECULAR SIEVE SUPPORT DESICCANT

AUTHORISI - J.E.HESS

382 FABR ICATION OF MULTILAYER PRINTED MIRING BOAROS IREV.) AUTHORISI - M.L.WILSON

386
(REV.) MOISTURE CONTENT STUDV

387
IREV.) DEVELOPMENT DF ELASTOMER CUSHIONS

388 A STUDY OF THE COMPRESSION SET CHARACTERISTICS OF CELLULAR SILICONE SUBJECTED TO ABNORMALLY HIGH STRAIN AUTHORISI - P.H.ALDRICH

390 A STUDY OF THE FUNCTIONAL CAPABILITIES OF AN AIR-BEARING COMPRESSION TEST

(REV.) FIXTURE

AUTHOR(S) - G.E.MARTINETTE

391 A STUOY OF THE FUNCTIONAL CAPABILITIES OF A TOTAL AREA LOADING COMPRESSION

$08 / 12 / 71$

(PEV.) TEST FIXTURE AUTHORIS) - G.E.MARTINETTE

392 LIQUIO PROCESSING OF MOLDEO DESICCANT

(REV.) AUTHORIS! - B.A.HEFNER

393 DEVELOPMENT OF ELASTOMER CUSHIONS, FYT1-3

$07 / 15 / 71$

$07 / 15 / 71$

$07 / 28 / 71$

$09 / 07171$

$07 / 28 / 71$

$03 / 29 / 71$

$09 / 26 / 72$

$08 / 12 / 71$

$08 / 29 / 71$

$08 / 20 / 71$

IREV.)

395 AUTHORISI - G.H.EDMAN

402

PERCUSSIVE-ARC VERSUS PULSE-ARC MELOING AUTHORISI - D.L.HOLLAR

(REV.)

ELECTRICAL CONTACT SURFACE TREATMENT

$04 / 13 / 72$

404 QUALITY DIVISION QUARTERLY

AUTHORISI - B.T.QUARTERLY.

$05 / 11 / 72$

405

THE BLENOING DF SEVEN LOTS OF MERgIMAMIDE L-425 FOR USE IN THE M300 ADHESIVE

$07 / 15 / 71$ AUTHOR(S) - G.K.BAKER

$07 / 15 / 71$ AUTHORISI - C.H.SHITH

$03 / 71$

$05 / 03 / 71$

$06 / 18 / 71$ 
BDX-613- TITLE AUTHORISI - J.H.KUEHME

410

LINING OF COMPRESSION SET TEST FIXTURES AUTHORISI - M.J.CHAIT

412 THE REFEAEMCE ELECTROOE AMO ITS USE IM DETERMIMIMG IMOIVIOUAL ELECTRODE POTENTIALS AUTHORISI - P.E.KLINGSPOAN

414 CVO SCALING STUDY

AUTHCRIS) - N.E.MILLER, P.F.STABLEIN, HR.

415

ELEMENTARY RELATIONSHIPS OF WORKER EFFICIENCY TO THE WORK ENVIRONMENT AUTHORISI - D.A.YOUNGBERG

422

ELECTROPOLISHING OF CHEMICAL VAPOR DEPOSITED TUMGSTEN

427 ELECTRICAL TERMIMATIONS: WELDED, BRALED NNO BONOED AUTHOR(S) - D.E.STITTSWORTH

ELECTROCHEMICAL STUDIES OF THE COLD-COMPEA ELECTROPLATIMG BATH AUTHOR(S) - M.J.NAPIER

431 ELECTRONIC ENGINEER ING QUARTERLY AUTHORISI - B.T.QQUARTERLY

$12 / 21 / 71$

$03 / 13 / 72$

MATERIAL AND PROCESS DEVELOPMENT OF POLYURETHANE FOAH. FYT1-4

$10 / 06 / 72$

485 (REV.) AUTHOR(S) - J.R.FENOER

493 DEVELOPMENT OF ELASTOMER CUSHIONS, FY71-4

$10 / 06 / 72$

(REV.) AUTHORISI - G.H.EOMAN

$11 / 10 / 71$ 
502 THIN INSULATOR SHAPES

IREV.I AUTHQRISI - J.C.OALY

505 SOLDER SPLATTER CAUSED BY OUTGASSING HOLES IN PRINTED MIRIMG BDARDS AUTHORTSI - M.E.EVANS, B.T.LAMPE, C.L.LONS, R.B.SNELT

$7 / 13 / 72$

SYNTHESIS OF TOLUENE OIISOC YAMATE DIMER ITOIDI.

AUTHORPSI - G.K.BAKER, B.C.COLE, R.K.JLUGLIMS, L.E.LACY, M.E.RICMARDSON AUTHOR(S) - R.L.EEGGS, C.J.CAMP

$09 / 20 / 71$

508 AN EVALUATION OF COMAERCIAL POLYAR POLVISOCVANATES IN A BENDIX KANSAS CITY FOAM SYSTEM AUTHOR(S) - J. SALARY

509 A DESCRIPTION OF VARIOUS METHOOS OF PREPAIMG POLYAMIC ACIOS ANO POLYIMIDES AUTHORTSI - D.R.ANSLEY, J.C.OALY, E.L. JOANSON

510 QUANTITATIVE ADJUSTMENTS FOR PERCENT OEFECTIVES IN NON-NORHAL OISTR IBUTIONS AUTHOR(S) - R.L.KIRKPATRICK

$09 / 07 / 71$

RAPID VISUAL SCANNING SYSTEMS FOR PCO'S, FYTI-2 AUTHORISI - R.D.TAYLOR

531 A STUDY OF POLYETHER-POLYOL-ANO POLYESTER-POLYOL-BASED RIGIO URETMANE FOAN SYSTEMS

AUTHORISI - G.K.BAKER, J.P.MMOEN, C.H.SHITH

$09 / 07 / 71$

$09 / 07 / 71$

$11 / 10 / 71$

$11 / 10 / 71$

$07 / 18 / 72$

$09 / 27 / 72$

$12 / 22 / 71$

$09 / 20 / 71$

$11 / 12 / 71$

$12 / 22 / 71$

$09 / 20 / 71$

$09 / 20 / 71$ AUTHOR (S) - J.C.DALY

535+ HOT GAS MOLOING OF POLYSTYRENE FOAM

$11 / 21 / 73$

$02 / 10 / 72$ AUTHOR(S) - D.L.RICE, G.F.MATKINS

conformai "cöting Fr $7 \mathrm{~T}^{-3}$ AUTHOR(S) - M.F.MOYLE

$11 / 12771$ AUTHOR $(S)$ - J.R.FENOER AUTHOR(S) - R.D. TAYLOR 
552 ULTRAPRECISION TOOL GRINDING

$09 / 27 / 72$ AUTHORISI - D.S.BARTEL

553 MATERIAL AND PROCESS DEVELOPMENT OF POL YURETHANE FOAM, FYT2-1

$10 / 06 / 72$

(REV.) AUTHOR(S) - J.R.FEHDER

559 RUST PREVENTION IN TOOL STORAGE AREAS

$11 / 21 / 73$

561 NONDESTRUCTIVE TESTING OF PERCUSSIVE ARC MELDS

$02 / 13 / 73$

(REV.) AUTHOR(S) - K.O.COX

562 MECHANICAL AND PHYSICAL PROPERTIES OF ORGANIC FOAM

$05 / 15 / 72$

(REV.I AUTHOR(S) - F.E.LORMIS

563 INSPECTION OF FINE-PITCHEO GEARS

$05 / 15 / 72$

(REV.) AUTHORISI - C.M.BERRY, JR.

564+ IN-LOT VARIATIONS IN POLYSTYRENE EXPAMOABLE BEAOS AMD BEAD FOAM AUTHOR(S) - R.M.PAL MER

$02 / 10 / 72$

576 MACHINING MINIATURE SLOTS AND FILLETS

$04 / 21 / 72$ AUTHORISI - L.K.GILLESPIE

577 STRAIN HARDENING EXPONENT FOR IT- PH STAINLESS STEEL AUTHORISI - J.R.MULKEY

$12 / 22 / 71$

578 VOLATILE LOSS IN POLYSTYRENE FOAM AUTHOR(S) - D.J.FOSSEY, C.E.ROEBUCK

$12 / 22 / 71$

579 AUTOCLAVE FABRICATION TECHNIQUES FOR FLAT CABLES

$07 / 13 / 72$

(REV.) AUTHOR(S) - J.D.SHEELEY

582 FABRICATION OF POLYIMIDE SYNTACTIC FOAM PARTS

$05 / 15 / 72$

585 BIBL IOGRAPHY: MACHINING MINIATURE PARTS AMO MINIATURE FEATURES

$01 / 26 / 72$ AUTHOR(S) - L.K.GILLESPIE

593 . DURURRING: A BIBLIOGRAPHY AUTHORISI - L.K.GILLESPIE

$01 / 26 / 72$

601 AN INVESTIGATION OF INTERNAL CAVITY PRESSURE FOR INJECTION-MOLDED

$07 / 24 / 72$

IREV.I POLYCARBONATES

602 HAND TOOL FOR INSTALLING SOLOER FORMS OVER COMPONENT LEADS

IREV.) AUTHORISI - D.W.BOWLEN

604 DETERMINATION OF SEMICONDUCTOR JUNCTION PROFILES BY ANGLE LAPPING OF

(REV.) SEMICONDUCTOR WAFERS

AUTHORIS) - E.L.RAUCH

607 HELICAL TAPE MIMOER

(REV.) AUTHOR(S) - J.O.ALEXANDER

613 ELECTRICAL TERMINATIONS: HELDED, ERALED ANO BONOED, FYT2-2

(REV.) AUTHOR(S) - D.E.STITTSWORTH

614

BEAM LEAO SEMTCONDÜCTOR TESTING AND FXICURE ANACYSIS

AUTHOR(S) - L.W.BROWN, L.H.SMITH

615 PROTECTIVE COATINGS FOR URANIUM-NIOBIUM HLOYS AUTHOR (S) - N.T.PANOUSIS

$03 / 15 / 72$

616 SOLDER LEVELING

$03 / 15 / 72$ AUTHOR (S) - H.A.MULHOLLAND

637 BENDIX KANSAS CITY ELECTRICAL TERMINATION MELDING HANDBOOK AUTHORIS) - D.E.STITTSWORTH, A.K.THOENI

$05 / 15 / 72$

THERMAL SHOCK DEBURRING

642

$07 / 11 / 72$

648 A SUSPENDEO METAL MASK FOR THICK-FILM PRINTIMG

$07111 / 72$

649 EFFECTS OF GEOMETRY ON THICK-FILM RESISTORS

$07 / 11 / 72$ AUTHOR(S) - G.A.SWARTL 


\begin{tabular}{|c|c|c|}
\hline $\begin{array}{l}\text { DOCUMENT } \\
\text { NUMBER } \\
\text { BDX-613- }\end{array}$ & TITLE & $\begin{array}{l}\text { OATE OF } \\
\text { RELEASE }\end{array}$ \\
\hline 650 & $\begin{array}{l}\text { PHOTOMASK MEASURING SYSTEMS } \\
\text { AUTHOR }(S) \text { - A. LAUDEL, JR. }\end{array}$ & $07 / 11 / 72$ \\
\hline 651 & $\begin{array}{l}\text { OEPOSIT ION OF CHROMIUM BY RAOIO FREQUENCY SPUTTERING } \\
\text { AUTMOR(S) - R.H.PIERCE. JR. }\end{array}$ & $07 / 11 / 72$ \\
\hline 652 & $\begin{array}{l}\text { PLATED-THROUGH HOLES BY PLASMA-EMHANCED DEPOSITION } \\
\text { AUTHORISI - A. LAUDEL. JR. }\end{array}$ & $07 / 11 / 72$ \\
\hline 653 & $\begin{array}{l}\text { PHOTORESIST CHARACTERIZATION METHOD FOR HYBRIO MICROCIRCUITS } \\
\text { AUTHOR(S) - L.E.SCHANTL }\end{array}$ & $07 / 11 / 72$ \\
\hline 654 & $\begin{array}{l}\text { INPUT AND OUTPUT DEVICES FOR A COMPUTERILED MASK-MAKING SYSTEM } \\
\text { AUTHORISI - L.R.SHEETS }\end{array}$ & $07 / 11 / 72$ \\
\hline 662 & $\begin{array}{l}\text { ELECTRICAL TERMINATIONS: MELDED, BRALED, AND BONDED, FYTZ-3 } \\
\text { AUTHOR(S) - D.E.STITTSHORTH }\end{array}$ & $07 / 13 / 73$ \\
\hline 664 & $\begin{array}{l}\text { REQUIREMENTS FOR REFLOW SOLDERING OF INTECRATEO CIRCUIT FLATPACKS } \\
\text { AUTHOR(S) - B.T.LAMPE }\end{array}$ & $04 / 72$ \\
\hline 667 & $\begin{array}{l}\text { FILAMENT HINDING INITIAL OEVELOPMENT } \\
\text { AUTHORISI - G.F.KORACH, R.H.PALMER }\end{array}$ & $04 / 21 / 72$ \\
\hline 670 & $\begin{array}{l}\text { PREVENTION OF TIN-LEAD OVERHAMD IN PLATED COPPER-CLAO PRINTED HIRING BOAROS } \\
\text { AUTHORISI - D.L.SWISHER }\end{array}$ & $03 / 06 / 73$ \\
\hline $\begin{array}{l}676 \\
\text { IPEV.1 }\end{array}$ & $\begin{array}{l}\text { SEMI-RIGID COAXIAL CVR/CABLE, FYT2-3 } \\
\text { AUTHORISI - K.D.ALMQUIST }\end{array}$ & $10 / 06 / 72$ \\
\hline 678 & $\begin{array}{l}\text { HYBR ID MICROCIRCUIT PHOTOLITHOGRAPHIC PROCESS } \\
\text { AUTHORISI - L.E.SCHANT } 2 \text {. }\end{array}$ & $09 / 19 / 73$ \\
\hline 687 & $\begin{array}{l}\text { CABLE FABR ICATION AND ENCAPSULATION PROCESS IMPROVEMENTS, FYT2-3 } \\
\text { AUTHORISI - T.J.HUSBY }\end{array}$ & $11 / 20 / 72$ \\
\hline $690 *$ & $\begin{array}{l}\text { HIGHDENSITY BRAID CABLE SHIELOS, FYT2-3 } \\
\text { AUTHORISI - J.F.GALBRAITH }\end{array}$ & $11 / 06 / 72$ \\
\hline $\begin{array}{l}692 \\
\text { (REV.) }\end{array}$ & $\begin{array}{l}\text { DRY MIXING DF MOLDED DESICCANT } \\
\text { AUTHORISI - H.J.NICHOLSON }\end{array}$ & $12 / 15 / 72$ \\
\hline $\begin{array}{l}697 \\
\text { IREV. I }\end{array}$ & $\begin{array}{l}\text { ROLAMITE SENSORS } \\
\text { AUTHORISI - C.H.BERRY, JR. }\end{array}$ & $02 / 16 / 73$ \\
\hline 703 & $\begin{array}{l}\text { LASER INTERFEROMETER SCALING COUNTER } \\
\text { AUTHOR (S) - L.D.WONNELL }\end{array}$ & $07 / 11 / 72$ \\
\hline 704 & $\begin{array}{l}\text { A QUANTITATIVE STUDY OF THE SPECTRAL DISTRIBUTION OF LOH ENERGY X-RAY } \\
\text { DEVICES } \\
\text { AUTHOR(SI - R.V.HECKMAN }\end{array}$ & $07 / 11 / 72$ \\
\hline $\begin{array}{l}705 \\
\text { (REV.) }\end{array}$ & $\begin{array}{l}\text { LOW TEMPERATURE PROPERTIES OF GENERAL ELECTRIC LEXAN B070-112 THIN } \\
\text { POLYCARBONATE FILM } \\
\text { AUTHORISI - L.J.LAZARUS }\end{array}$ & $10 / 06 / 72$ \\
\hline $\begin{array}{l}\text { TOB } \\
\text { (REV.) }\end{array}$ & $\begin{array}{l}\text { OPTIMIZING MINIATURE CYLINDRICAL PIECEPART MACHINING CAPABILITIES } \\
\text { AUTHOR(S) - J.M.ROBB }\end{array}$ & $08 / 03 / 73$ \\
\hline $712+$ & $\begin{array}{l}\text { FABR ICATION OF FATITSAFE PQESSURE VESSEELS } \\
\text { AUTHORISI - M.C.RUTHERFORD }\end{array}$ & $06 / 24774$ \\
\hline $\begin{array}{l}\text { T13 } \\
\text { (REV.) }\end{array}$ & $\begin{array}{l}\text { SURFACE ENERGETICS OF CONFORMAL COATIMGS } \\
\text { AUTHORISI - L.C.JACKSON }\end{array}$ & $05 / 04 / 73$ \\
\hline $\begin{array}{l}716 \\
\text { (REV.) }\end{array}$ & $\begin{array}{l}\text { QUALIFICATION OF MINIATURE INTERMAL THREADS USING AIR FLOW } \\
\text { AUTHOR(S) - C.H.BERRY, JR. }\end{array}$ & $05 / 04 / 73$ \\
\hline $718 *$ & $\begin{array}{l}\text { MATERIAL AND PROCESS DEVELOPMENT OF POLYURETHAME FOAM, FYT2-3 } \\
\text { AUTHORISI - J.R.FENDER }\end{array}$ & $03 / 08 / 73$ \\
\hline 7204 & $\begin{array}{l}\text { AN EXPLOOING BR IDGEWIRE SIMULATOR USING THE SCEPTRE CIRCUIT ANALYSIS PROGRAM } \\
\text { AUTHORTSI - J.R -LEMBRE }\end{array}$ & $08 / 24 / 72$ \\
\hline 728 & $\begin{array}{l}\text { TRIMMABLE FILN RESISTORS } \\
\text { AUTHORISI - T.F.BARNARD }\end{array}$ & $11 / 20 / 72$ \\
\hline
\end{tabular}




\begin{tabular}{|c|c|c|}
\hline $\begin{array}{l}\text { DOCUMENT } \\
\text { NUMBER } \\
\text { BDX-613- }\end{array}$ & TITLE & $\begin{array}{l}\text { DATE OF } \\
\text { RELEASE }\end{array}$ \\
\hline $\begin{array}{l}735 \\
\text { (REV.) }\end{array}$ & $\begin{array}{l}\text { VIBRATORY DEBURRING } \\
\text { AUTHORISI - L.K.GILLESPIE }\end{array}$ & $03 / 29 / 74$ \\
\hline 738 & $\begin{array}{l}\text { NONMETALLIC TOOLING CAPABILITIES AND ECONOMICS, FYT2-4 } \\
\text { AUTHORISI - R.W.FREUND }\end{array}$ & $11 / 20 / 72$ \\
\hline 739 & $\begin{array}{l}\text { SOLDER LEVEL ING } \\
\text { AUTHORISI - W.A. MULHOLLANO }\end{array}$ & $12 / 20 / 72$ \\
\hline 740 & $\begin{array}{l}\text { CABLE FABR ICATION AND ENCAP SULATION PROCESS IMPROVEMENTS, FYT2-4 } \\
\text { AUTHORISI - T.J.HUSBY }\end{array}$ & $11 / 20 / 72$ \\
\hline 742 & $\begin{array}{l}\text { FLUID FLOW ANALYSIS IN THE MOLD MEATIMG MO COOLING LOOP } \\
\text { AUTHORISI - R.H.FLOERSCH }\end{array}$ & $11 / 20 / 72$ \\
\hline $\begin{array}{l}760 \\
\text { (REV.) }\end{array}$ & $\begin{array}{l}\text { THIN-FILM HYBRID MICROC IRCUIT SUBSTRATE KETALLILATION } \\
\text { AUTHORISI - N.V.FORTE }\end{array}$ & $11 / 21 / 73$ \\
\hline 767 & $\begin{array}{l}\text { A DIGITAL FREQUENCY MEASURING SYSTEM FOR PULSED RF OEVICES } \\
\text { AUTHORISI - L.C.BEOUGHER }\end{array}$ & $08 / 28 / 72$ \\
\hline $769+$ & $\begin{array}{l}\text { DYNAMIC TESTING OF EXPLODING BR LOGEWIRES } \\
\text { AUTHOR(S) - J.R.LEMBKE }\end{array}$ & $01 / 12 / 72$ \\
\hline 773 & $\begin{array}{l}\text { ELECTRICAL TERMINATIONS: MELDED, BRAZED, AMD BOMDED, FYT2-4 } \\
\text { AUTHORISI - D.E.STITTSWORTH }\end{array}$ & $05 / 04 / 73$ \\
\hline 775 & $\begin{array}{l}\text { CABLE FABR ICATION ANO ENCAP SULATION, FVT3-I } \\
\text { AUTHORISI - T.J.HUSBY }\end{array}$ & $03 / 06 / 73$ \\
\hline $\begin{array}{l}776 \\
\text { (REV.) }\end{array}$ & $\begin{array}{l}\text { HIGH-DENSITY GRAID CABLE SHIELDS, FYT2-4 } \\
\text { AUTHOR(S) - H.J.EDWARDS, JR. }\end{array}$ & $03 / 06 / 73$ \\
\hline 778 & $\begin{array}{l}\text { NONMETALLIC TOOLING CAPABILITIES ANO ECONOMICS, FYT3-1 } \\
\text { AUTHOR (S) - R.H.FREUND }\end{array}$ & $03 / 23 / 73$ \\
\hline 779 & $\begin{array}{l}\text { BEAM LEAD DEVICES AND THE HMC MANUFACTURER } \\
\text { AUTHOR S }=\text { R.E.MARKLEY }\end{array}$ & $10 / 06 / 72$ \\
\hline 783 & $\begin{array}{l}\text { INVESTIGATION OF KNITTEO FABRICS } \\
\text { AUTHOR(S) - R.F.TAYLOR }\end{array}$ & $10 / 22173$ \\
\hline $791+$ & $\begin{array}{l}\text { FINAL REPORT ON MOTION SENSORS } \\
\text { AUTHORISI. - H.C.MONDAY, Y.V.SMITH }\end{array}$ & $10 / 24 / 72$ \\
\hline $\begin{array}{l}793 \\
\text { (REV.) }\end{array}$ & $\begin{array}{l}\text { CLEANING AND ASSEMBLY TECMNLQUES, FYT3-1 } \\
\text { AUTHOR(S) - M.L.WADOELL }\end{array}$ & $02 / 16 / 73$ \\
\hline 797 & $\begin{array}{l}\text { MATER IAL AND PROCESS DEVELOPMENT OF POLYURETHAME FOAM, FYT3-1 } \\
\text { AUTHOR (S) - J.R.FENDER }\end{array}$ & $05 / 21 / 73$ \\
\hline 804 & $\begin{array}{l}\text { NUMERICALLY-CONTROLLED ASSEMBLY } \\
\text { AUTHORISI - R.H.HUGHS }\end{array}$ & $02 / 16 / 73$ \\
\hline 805 & $\begin{array}{l}\text { GOLO PLATING } \\
\text { AUTHORIS) - C.J.STIMETL }\end{array}$ & $05 / 21 / 73$ \\
\hline 812 & $\begin{array}{l}\text { TESTING FOR MATERIAL FLAWS ANO THERMAL DESIGN DEFECTS USING HQLOGRAPHIC } \\
\text { INTERFEROMETRY } \\
\text { AUTHOR(S) - WOP.SCHOEPPNER }\end{array}$ & $12 / 15 / 72$ \\
\hline 813 & $\begin{array}{l}\text { NONLINE A COMPUTER PROGRAM FOR LEAST SQUAMES ESTIMATION OF MONLINEAR } \\
\text { PARAMETERS AND APPLICATIONS } \\
\text { AUTHORISI - B.J.NEAL }\end{array}$ & $03 / 06 / 73$ \\
\hline 814 & $\begin{array}{l}\text { DETERMINATION OF HOLE LOCATION ON PRINTEO CIRCUIT BOARDS } \\
\text { AUTHORIS) - R.D.TAYLOR }\end{array}$ & $03 / 06 / 73$ \\
\hline 8.15 & $\begin{array}{l}\text { JUNCTION FIELD EFFECT TRANSISTOR DEGRAOATION CAUSED BY ELECTROSTATIC } \\
\text { DISCHARGE } \\
\text { AUTHORISI - W.J.KIRK, JR. }\end{array}$ & $12 / 22 / 72$ \\
\hline $\begin{array}{l}820 \\
\text { (REV.) }\end{array}$ & $\begin{array}{l}\text { LASER REMOVAL OF DIELECTRIC FROM FLAT CABLES } \\
\text { AUTHOR }(S) \text { - R.T.HORN }::\end{array}$ & $04 / 24 / 74$ \\
\hline $\begin{array}{l}821 \\
\text { (REV.) }\end{array}$ & $\begin{array}{l}\text { MOISTURE EFFECTS ON THERMOSET MOLDING COMPOUNOS } \\
\text { AUTHOR(S) - J.M.CONHAY }\end{array}$ & $03 / 06 / 73$ \\
\hline $\begin{array}{l}824 \\
\text { IREY }\end{array}$ & $\begin{array}{l}\text { THE CREATION OF LARGE CASTIMGS BY THE INVESTMENT PROCESS } \\
\text { AUTMOR(S) - M.E.CROMHELL }\end{array}$ & $03 / 06 / 73$ \\
\hline
\end{tabular}




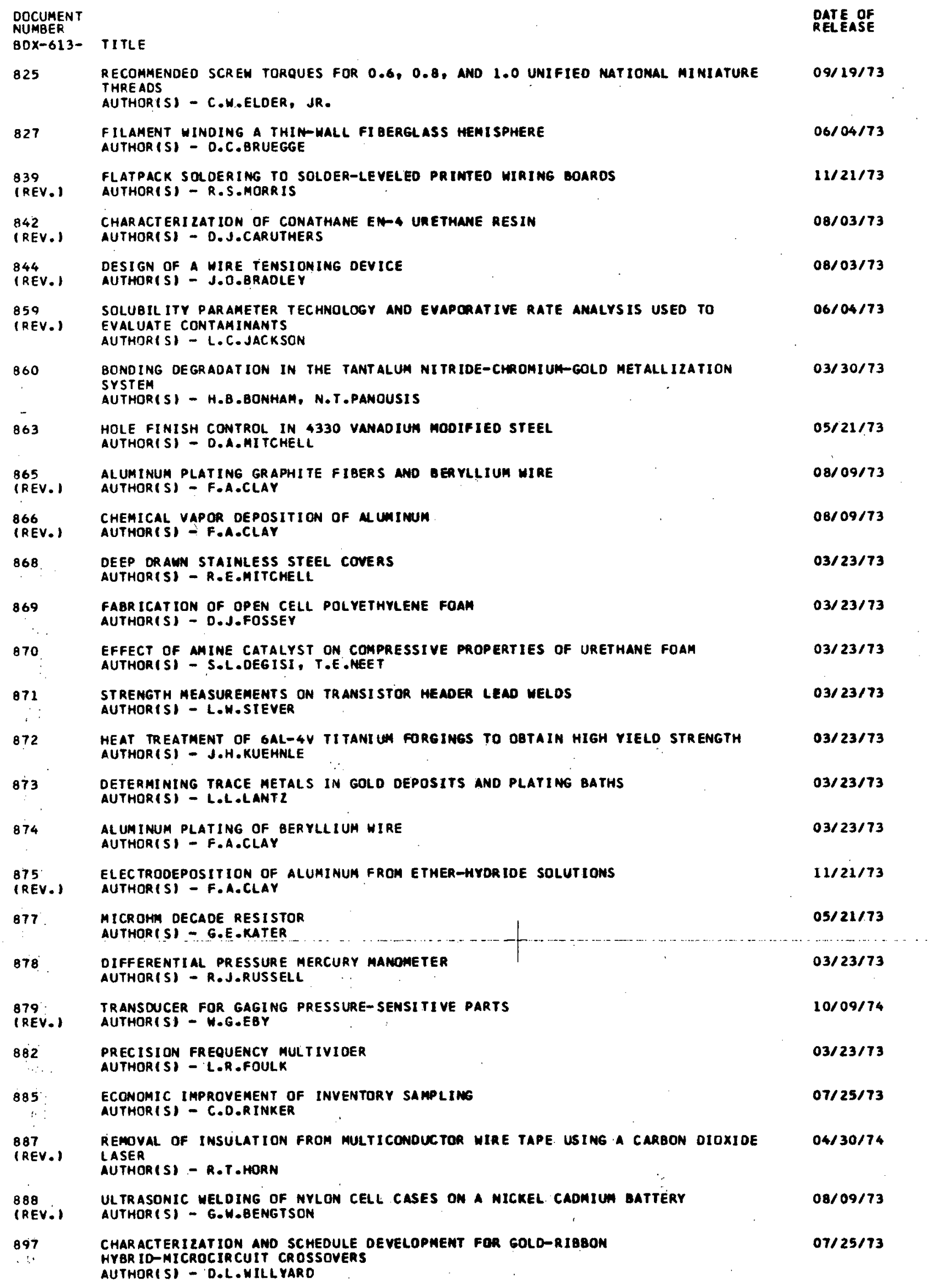




\begin{tabular}{|c|c|c|}
\hline $\begin{array}{l}\text { DOCUMENT } \\
\text { NUMBER } \\
\text { BDX-613- }\end{array}$ & $\cdots$ & $\begin{array}{l}\text { DATE OF } \\
\text { RELEASE }\end{array}$ \\
\hline 904 & $\begin{array}{l}\text { LINEAR TRANSDUCER TIP PRESSURE CHECKER } \\
\text { AUTHORISI - E.B.COOPER }\end{array}$ & $07 / 13 / 73$ \\
\hline 909 & $\begin{array}{l}\text { CABLE FABR ICATION AND ENCAP SULATION PROCESS IMPROVEMENTS, FYT3-3 } \\
\text { AUTHOR } S S \text { - T.J.HUSBY }\end{array}$ & $08 / 30 / 73$ \\
\hline $\begin{array}{l}910 \\
\text { (REV.) }\end{array}$ & $\begin{array}{l}\text { IMPROVED DENSITY CONTROL OF MOLOED DESICCANTS } \\
\text { AUTHORISI - J.M.CONHAY }\end{array}$ & $08 / 30 / 73$ \\
\hline 914 (REV.) & $\begin{array}{l}\text { WOVEN-WIRE TAPE VENDOR DEVELOPMENT FYT3-3 } \\
\text { AUTHORISI - D.H.CHAMBERS }\end{array}$ & $09 / 19 / 73$ \\
\hline 921 & $\begin{array}{l}\text { OELIQUESCENT-CHEMICAL MAXIMUM HUMIOITY INOICATOR } \\
\text { AUTHOR(S) - W.B.ABEL }\end{array}$ & $06 / 04 / 73$ \\
\hline 926 & $\begin{array}{l}\text { VIBRATION ANALYSIS } \\
\text { AUTHORISI - C.H.BERRY, JR. }\end{array}$ & $12 / 12 / 73$ \\
\hline $\begin{array}{l}927 \\
\text { IREV.I }\end{array}$ & $\begin{array}{l}\text { OEVELOPMENT OF A SELF-CONTAINED LAMINATING FIXTURE } \\
\text { AUTHORISI - J.O.SHEELEY }\end{array}$ & $05 / 29 / 74$ \\
\hline $\begin{array}{l}930 \\
\text { (REV.) }\end{array}$ & $\begin{array}{l}\text { EFFECTS OF MOISTURE UPON SP IRAL FLOW IN THERMOSET MOLOING COMPOUNDS } \\
\text { AUTHOR(S) - J.M.CONHAY }\end{array}$ & $08 / 03 / 73$ \\
\hline (REV.) & $\begin{array}{l}\text { PROTECTION OF GLASS TUBES IN EPOXY ENCAPSULATION } \\
\text { AUTHORISI - J.H.STEELE }\end{array}$ & $03 / 29 / 74$ \\
\hline 932 & $\begin{array}{l}\text { PANTOGRAPH POSITIONING SYSTEM FOR INSPECTING MICROCIRCUITS } \\
\text { AUTHORISI - R.D.TAYLOR }\end{array}$ & $07 / 25 / 73$ \\
\hline 933 & $\begin{array}{l}\text { LASER CENTER ING DETECTOR } \\
\text { AUTHOR }(S) \text { - L.D. HONNELL }\end{array}$ & $08 / 03 / 73$ \\
\hline 936 & $\begin{array}{l}\text { JOINING MAGNETIC ALLOY 49FE-49CO-2V TO } 304 L \text { STAINLESS STEEL } \\
\text { AUTHOR(S) - O.M.JARBOE }\end{array}$ & $08 / 03 / 73$ \\
\hline 938 & $\begin{array}{l}\text { LOW DENSITY FOAMS FOR VACUUM INSULATION } \\
\text { AUTHOR(S) - W.E.RICHARDSON }\end{array}$ & $11 / 21 / 73$ \\
\hline 941 & $\begin{array}{l}\text { RISE-RATE TESTING OF URETHANE FOAMS } \\
\text { AUTHORISI - T.E.NEET }\end{array}$ & $08 / 30 / 73$ \\
\hline 942 & $\begin{array}{l}\text { OMNI-DIRECTIONAL ILLUMINATION FOR PRINTED CIRCUIT BOARD INSPECTION } \\
\text { AUTHORISI - R.D.TAYLOR }\end{array}$ & $08 / 03 / 73$ \\
\hline $\begin{array}{l}944 \\
\text { (REV.) } \\
\vdots\end{array}$ & $\begin{array}{l}\text { IMPACT TESTING OF MATER IALS USING AN EIGHT-IMCH AIR GUN. AND COMPUTER } \\
\text { REDUCTION OF DATA } \\
\text { AUTHORISI - L.F.THORNE }\end{array}$ & $11 / 21 / 73$ \\
\hline 946 (REV.) & $\begin{array}{l}\text { IMPACT TESTING OF FOAM-FILLLD PAPER HOMENCOMB WITH ENERGY-ABSORPTION } \\
\text { CHARACTERISTICS COMPAREO TO. BALSA AND REDWOOD } \\
\text { AUTHORISI - R.D. JUMP, L.F. THORNE }\end{array}$ & $05 / 07 / 74$ \\
\hline 9.48 & $\begin{array}{l}\text { EXTRUDE HONE DEBURRING } \\
\text { AUTHOR(S) - L.K.GILLESPIE, L.H.LUEBBERT, JR. }\end{array}$ & $08 / 09 / 73$ \\
\hline 951 & $\begin{array}{l}\text { PRESSURE DISTRIBUTION IN POLYCARBONATE INJECTION MOLDINGS } \\
\text { AUTHORISI - P.C.BLACK }\end{array}$ & $12 / 12 / 73$ \\
\hline 952 & $\begin{array}{l}\text { LINEAR THERMAL EXPANSTON OF FILLED EPOXY RESINS } \\
\text { AUTHORISI - H.M.MCILROY }\end{array}$ & $05 / 09774$ \\
\hline $\begin{array}{l}9,53+ \\
\vdots\end{array}$ & $\begin{array}{l}\text { IMPROVED CUR VEO VACUUM BLANKETS FOR PHOTORESIST EXPOSURE } \\
\text { AUTHOR(S) - W.H.KNOPF }\end{array}$ & $06 / 24 / 74$ \\
\hline 955 & $\begin{array}{l}\text { ALUMINUM ETCHANT CONTROL STUDY } \\
\text { AUTHOR }(S) \text { - M.H.KNOPF }\end{array}$ & $11 / 21 / 73$ \\
\hline 956 & $\begin{array}{l}\text { LIGHT EMITTING OIODE TESTING AND ANALYSIS } \\
\text { AUTHOR (S) - J.L.BOWERS }\end{array}$ & $08 / 03 / 73$ \\
\hline $\begin{array}{l}959 \\
\text { (REV.) }\end{array}$ & $\begin{array}{l}\text { MASK GENERATION } \\
\text { AUTHOR(S) - L.E.SCHANTZ }\end{array}$ & $11 / 21 / 73$ \\
\hline $\begin{array}{l}960 \\
\text { (REV.) }\end{array}$ & $\begin{array}{l}\text { INVESTIGATION OF POLYSULFIOE CONFORMAL COATING } \\
\text { AUTHORISI - C.J.CAMP }\end{array}$ & $03 / 29 / 74$ \\
\hline 964 & $\begin{array}{l}\text { IMPROVED WORK DIRECTORS } \\
\text { AUTHOR(S) - R.C.CARTER }\end{array}$ & $11 / 21 / 73$ \\
\hline
\end{tabular}




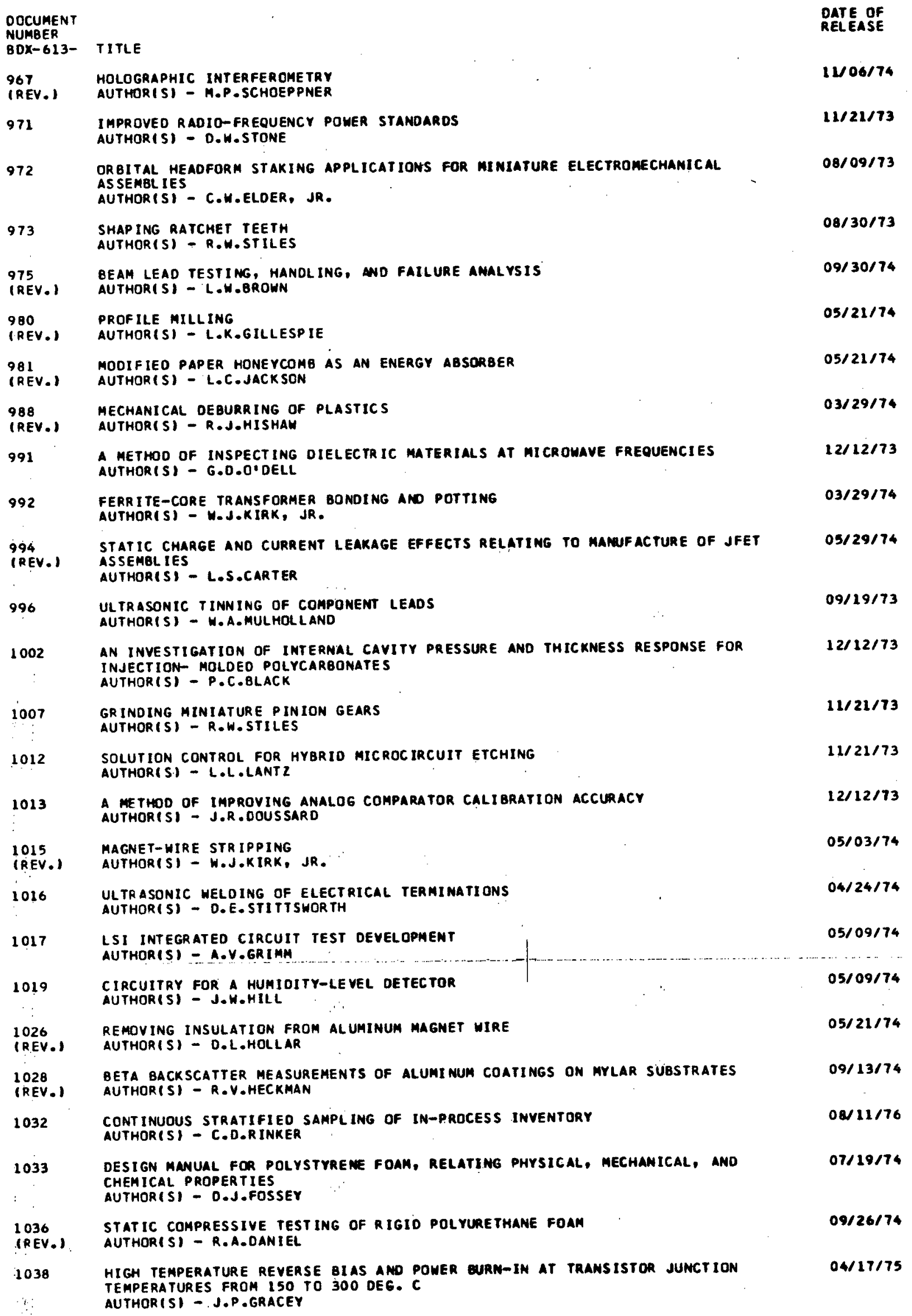


1044 NON-DESTRUCTIVE METHOOS FOR THE ACCEPTANCE OF PLATING THICKNESS DN PRINTED

$04 / 29 / 74$ CIRCUIT BOARDS AUTHORIS) - W.B.ABEL.

1046 aUTOMATIC PEAK POHER MEASUREMENT OF SHORT RF PULSES

$08 / 15 / 74$ AUTHOR (S) - K.E.HAROINGER, E.E.PETER SON

1047 THE EFFECT OF CUTTING EOGE RADIUS ON POISSON BUR PROPERTIES AUTHORISI - L.K.GILLESPIE

$11 / 26 / 73$

1048 COMPARISON OF METHOOS FOR CLEANING CONTAMIMATED SURFACES

(REV.) AUTHOR(S) - L.C.JACKSON

1049 STEEL-RULE DIE CONTOURING EVALUATION AUTHORISI - R.A.HILE

1051 FEASIBILITY OF ORAG SOLDERING

IREV.) AUTHORISI - R.S.MORRIS

1053 READ-ONLY MEMORY IMPLEMENTATION STUDY

(REV.) AUTHOR(S) - J.D.ANDERSON

1056 BRUSH DEBURRING OF MINIATURE PARTS

IREV.) AUTHOR(S) - L.K.GILLESPIE

1059 TECHNIQUE FOR IMPACT TESTING OF CONF INEO RIGIO FOAM

(REV.) AUTHOR(S) - R.D.JUMP, L.F.THORNE

1061 SPATIAL DISTRIBUTION FROM SMALL BETA SOURCES AND THE EFFECTS OF APERTURE SIZE AUTHOR(S) - R.V.HECKMAN

1062 A SOL ID STATE CIRCUIT BREAKER AUTHOR (S) - E.R.ELMER

1064

(REV.)

ELECTRODEPOSITING PT AND PT-IR ALLOYS FROM MOLTEN CYANIDES: CHEMICAL PROCESS STUDIES

AUTHOR(S) - M.B.HARDING

1065

MONMETALLIC TOOLING CAPABILITIES AND ECOMOMICS

$11 / 26 / 73$

$05 / 07 / 74$

$05 / 07 / 74$

$05 / 03 / 74$

$08 / 20 / 74$

$20 / 09 / 74$

$06 / 14 / 75$

$03 / 29 / 74$ AUTHOR(S) - R.W.FREUND

1066 TEN-BIT SAMPLE AND HOLD OR PEAK HOLD CONVERTER AUTHORISI - S.E.GRANT

1067 INSULATION REMOVAL FROM WOVENWWIRE TAPE

(REV.) AUTHORIS) - R.L.NANEY

IO68 FLEXCIRCUIT PROTECTIVE COATINGS
(REV

(REV.)

MINI ATURE-INTERCONNECTION PROCE SSING

$\begin{array}{ll}1069 & \text { MINIATURE-INTERCONNECTIOR } \\ \text { (REV.) } & \text { AUTHOR(S) - D.L.HOLLAR }\end{array}$

1076

AUTOMATED VISUAL INSPECTION SYSTEM FOR PRINTED MIRING ASSEMBLIES

(REV.)

AUTHOR(S) - R.D.TAYLOR

1077 AUTOMATED SCHEDULING OF MANUFACTUR ING RESOURCES

AUTHOR (S) - H.G.HOPPEN

(

RESOURCES FORECASTING SYSTEM AUTHOR(S) - J.J.KOLICH

1079 APPLICATION OF STATISTICAL METHOOS TO MAMUFACTURING OPERATIONS AUTHORISI - J.H.CARDER, C. LATIMER

IOBO CONTROL OF ELECTROSTATIC DAMAGE TO SOLID STATE DEVICES AUTHOR (S) - L.S.CARTER, M.J.KIRK, JR.. M.L.HAODELL

IOB 1 AUTOMATIC TESTING ANO HANDL ING OF BEAM LEAD TRANSISTORS ANO DIODES AUTHOR(S) - L.H.SMITH, B.G. WILSON

L.082 PROCESS EVALUATION OF NEW CELLULAR SILICONE MATERIAL

(REV.) AUTHOR(S) - G.H.EDMAN

1.083

THE EFFECTS OF REAMING VARIABLES ON BURR PROPERTIES

$04 / 30 / 74$

$04 / 30 / 74$

$04 / 30 / 74$

$05 / 21 / 74$

$10 / 09 / 74$

$04 / 24 / 74$

$05 / 03 / 74$

$05 / 29 / 74$ 
1084 PRÓPERTIES OF BURRS PRODUCED BY BALL BROACHING

(REV.) AUTHORIS) - L.K.GILLESPIE

$04 / 17 / 75$

1085

THE EFFECT OF RAPIOLY CHAMGING VOLTAGES ON THICK-FILM RESISTORS

$04 / 30 / 74$

IREV.I

AUTHORISI - A.G.BENNETT

1086 PHOTOMULTIPLIER TUBE TESTIN: AND AMMLYSIS

AUTHORISI - J.L.BOWERS

$04 / 24174$

1099 SOLUBILITY PARAMETERS ANO EVAPORATIVE RATE aNALYSIS IN ORgANIC RESIDUE

$09 / 17 / 74$

(REV.) CHARACTERILATION

AUTHOR(S) - L.C.JACK SON

1101 AUTOMATIC PHOTOMASK ALIGNMENT AND EXPOSURE OM 3.75 BY 4.50 INCH SUBSTRATES

(REV.) AUTMORISI - L.E.SCHANTL

1103 PREOICTIMG COMPRESSIVE STRENGTHS IN URETHAME FOAMS AT HIGH STRAINS

IREV.) AUTHORISI - T.E.NEET

1104 DEVELOPMENT OF A LIGHT LOAD FRICTIOA TESTER

AUTHORISI - G.W.FORMAN, D.P.POPE

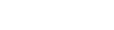

$03 / 14 / 75$

$05 / 03 / 74$

$05 / 01 / 74$

1105 ALUMINUM ETCHANT STUDIES

(REV.) AUTHOR(S) - M.M.KNOPF

1109 CHARACTERIZATION OF A CHROMIUH GOLO DEPOSITIOM PROCESS FOR THE PRODUCTION OF THIN FILM HYBRID MICROCIRCUITS AUTHORISI - F.A.CLAY, R.H.PIERCE, JR.

1110 POLYURE THANE/IMIOE MODIFIED FOAM AUTHORISI - J. SALARY

1111 EXPER IMENTAL TESTING FOR MOLD TEMPERATURE RESPONSES

(REV.) AUTHORISI - R.H.FLOERSCH

1112 THE BURR--MANUFACTUR ING 'S PERENNIAL THORN AUTHORISI - L.K.GILLESPIE

$09 / 30 / 74$

1113 BEAM LEAD DEVICE AND HYBRID MICROCIRCUIT TESTING AUTHOR(S) - R.E.MAR KLEY

1115 DEVELOPMENT OF PROCESSES FOR HMRIO MICROCIRCUITS CONTAINING BEAM-LEAO

(REV.) DEVICES AUTHOR(S) - R.J.BLAZEK

1116 EARLY PROOUCTION HISTORY ON HYBRID MICROCIRCUITS CONTAIMING BEAM-LEAD

(REV.) DEVICES AUTHOR(S) - T.A.WILEY

IIIT STATE-OF-THE-ART OF GENERAL. DEBURRING METHODS AUTHOR (S) - N.S.AJLOUNEY, L.K.GILLESPIE

1120 THE MEASUREMENT OF BURRS AUTHOR(S) - L.K.GILLESPIE

$05 / 01 / 74$

1121 PREPARATION OF A CTBN-MODIFIEO EPOXY RESIN

$11 / 06 / 74$

(REV.) AUTHOR(S) - M.E.RICHARDSON

$10 / 09 / 74$

1122
IREV DEFECT DETECTION IN HIEH

1123 DEVELOPMENT OF A FORMING STATION

IREV.I AUTHORISI - J.D.SHEELEY

$07 / 26 / 74$

1124 DEBURRING: AN ANNOTATEO BIBLIOGRAPHY

$05 / 01174$ AUTHOR(S) - L.K.GILLESPIE

1126. COST SAVING THROUGH APPLICATION OF THE INVESTKENT. CASTIMG PROCESS

(REV.) AUTHORISI: - W.E.CROMMELL

1130 ROUNO-HIRE LAMIMATEO FLAT CIRCUITS AUTHOR(S) - R.A.HILE

$08 / 11 / 76$

$08 / 20 / 74$

1133 TEMPERATURE DEPENDENT PROPERTIES OF POTTIMG MATERIALS

(REV.) AUTHORISI - J.M.HALKER

1134 AUTOMATIC RADAR-SENSITIVITY MEASUREMENT

(REV.) AUTHOR(S) - K.E.HARDINGER

$09 / 17 / 74$

1135 USE OF EN-7 TO ENCAPSULATE AMALYZER ASSEMBLIES

(REV.) AUTHORIS) - R.E.SCHMIDT

$09 / 13 / 74$ 
1138 AUTOMATED TESTING IN A HIGH NOI SE ENVIROMMENT

$06 / 03 / 74$

1140 RIGID URETHANE FOAM BASED ON METHYL-ALPHA-(D)-GLUCOSIOE AMD E-CAPROLACTONE

(REV.) AUTHORISI - J.H.MCFARLANO

$11 / 06 / 74$

1144

(REV.)

DETECTION AND SUPPRESSION OF TRANSISTOR OSCILLATION DURING POMER-BURN-IN AUTHORISI - R.H.SCHMIDT

$09 / 13 / 74$

1145 WORK-IN-PROCESS INVENTORY

$06 / 03 / 74$

, AUTHOR (S) - M.J.KEPNER

1148 SPRAY PHOTORESIST DEVELOPMENT ON MULTIPLE HYBRIO MICROCIRCUIT SUBSTRATES QUTHORISI - L.E.SCHANT Z

$05 / 12 / 75$

1150 MAXIMUM HUMIDITY INDICATOR

(REV.) AUTHORIS) - M.B.ABEL

1154 EFFECTS OF 8ORIC ACIO ON THE CURE OF OOROW-FILLED U-3602 SILICONE GUM

(REV.) AUTHOR(S) - G.E.LEICHL ITER

1155 MASS ANODILATION OF TANTALUM-NITRIOE RESISTORS

(REV.) AUTHORIS) - A.J.PELTIER

1158 ULTRASONIC MELDING OF ELECTRICAL TERMIMATIONS

(REV.) AUTHORIS) - G.S.DARNER

1159 ELECTROCHEMICAL GRINOING AUTHOR(S) - R.H.STILES

1167 A RAPID TCR TEST METHOD FOR TANTALUM-NITRIDE RESISTIVE FILM

(REV.) AUTHORISI - A. LAUDEL, JR.

1169 AUTOMATIC MACHINE APPLICATION OF SOL10 FILM LLORICANT AUTHOR(S) - G.P.MILLER

1170 LIGHT LOAD FRICTION COEFFIC IENT AND MEAR STUDY OF THAEE BONDED SOLIO LUBR ICANTS AUTHOR(S) - D.P.POPE

1171 EFFECTS OF THERMAL EXPANSION ON EPOXY GLASS LAMINATES AUTHOR(S) - E.E.COMBS

1172 SOLVENT ANALYSIS OF STRESSES IN MOLDED POLYCARBONATE PARTS AUTHOR(S) - C.L.HALTER

1173 CHARACTERIZATION OF A CHROME-GOLO THIN FILM DEPOSITIOM PROCESS BY AUGER ANAL YSIS

AUTHOR(S) - F.A.CLAY

1174 REFLECTEO LIGHT INTENSITY DISTR IBUTIONS FRON OEFECTS ON HIGHY REFLECTIVE

IREV.) SPHERES

AUTHOR(S) - P.E.KLINGSPCRN

1175 CHARACTERIZATION OF THERMOCOMPRESSION BONOING PARAMETERS FOR BEAM-LEAD BOND ING

AUTHOR(S) - B.H.LENHAAROT, D.AATYOUNTEOERG

1176

REMOVAL OF HYBR IO MICROCIRCUIT BEAM-LEAD DEVICES FOR POSTMORTEM TESTS AUTHOR(S) - B.W.LENHAROT, D.A.YOUNGBERG

1 II77 PLATING ON POLYIMIDE

(REV.) AUTHORISI - C.J.STIMETL

1178 EVALUATION OF A HIGH TEMPERATURE SYNTACTIC FOAM

(REV.) AUTHOR(S) - H.M.MCILROY

1180 WIRE WOVEN TAPE MEASUREMENTS

AUTHOR(S) - C.W.BERRY, JR.

$0.8 / 20 / 74$

$08 / 08 / 74$

$03 / 03 / 77$

$06 / 24 / 74$

1181 EFFECTS OF COATING AND CLEANING ON CORONA AND HIGH VOLTAGE BREAKDONM IN

(REV.) ELECTRONIC ASSEMBLIES AUTHOR(S) - E.R.BROWN

1182 AN ACTIVE LASER TRIMMER FOR PRODUCTION TRIMMING OF FUNCTIONAL HYBRID MICROCIRCUITS AUTHORISI - J.A.PETERS

$08 / 15 / 74$

$07 / 26 / 74$

$02 / 12 / 75$

$03 / 14 / 75$

$06 / 24 / 74$

MICROCIRCUITS
AUTHORISI - J.A.PETERS




\begin{tabular}{|c|c|c|}
\hline $\begin{array}{l}\text { DOCUMENT } \\
\text { NUMBER } \\
\text { BOX-613- }\end{array}$ & TITLE & $\begin{array}{l}\text { DATE OF } \\
\text { RELEASE }\end{array}$ \\
\hline 1187 & $\begin{array}{l}\text { RAPID VISUAL SCANNING } \\
\text { AUTHORISI - R.O.TAYLOR }\end{array}$ & $08 / 06 / 76$ \\
\hline 1189 & $\begin{array}{l}\text { EVALUATION OF THERMOPLASTIC POL YESTER MOLDING RESINS } \\
\text { AUTHOR(S) - C.L.WALTER }\end{array}$ & $07 / 22 / 75$ \\
\hline $\begin{array}{l}1190 \\
\text { (REV.) }\end{array}$ & $\begin{array}{l}\text { NONDESTRUCTIVE TESTING OF WELDED ELECTRICAL CONNECTORS } \\
\text { AUTHORISI - E.B.COOPER }\end{array}$ & $08 / 06 / 76$ \\
\hline $\begin{array}{l}1192 \\
\text { (REV.) }\end{array}$ & $\begin{array}{l}\text { FATIGUE FAILURE IN BRAIDED ELEC TROMAGNETIC-RADIATION SHIELDING } \\
\text { AUTHOR (S) - H.J-EOWARDS, JR. }\end{array}$ & $03 / 13 / 75$ \\
\hline $\begin{array}{l}1193 \\
\text { (REV.) }\end{array}$ & $\begin{array}{l}\text { PROCESSING OF MINIATURE INTERCONHECTIONS } \\
\text { AUTHORISI - D.L.HOLLAR }\end{array}$ & $11 / 06 / 74$ \\
\hline $\begin{array}{l}1199 \\
\text { (REV.) }\end{array}$ & $\begin{array}{l}\text { HIGHDENSITY POLYSTYRENE BEAD PREPARATION } \\
\text { AUTHORISI - D.L.RICE }\end{array}$ & $09 / 30 / 74$ \\
\hline 1200 & $\begin{array}{l}\text { CONTAMINANT REMOVAL USING SOLUBILITY PARAMETER TECHNOLOGY } \\
\text { AUTHORISI - L.C.JACKSON }\end{array}$ & $08 / 08 / 74$ \\
\hline $\begin{array}{l}1205 \\
\text { (REV.) }\end{array}$ & $\begin{array}{l}\text { EVALUATION OF SYNTACTIC POLYSULFIDE STRESS RELIEF COATINGS } \\
\text { AUTHORISI - T.E.NEET }\end{array}$ & $08 / 11 / 76$ \\
\hline 1207 & $\begin{array}{l}\text { COMPARISON OF ALTERNATE MATERIALS FOR MOCA CURED AOIPRENE L- } 100 \\
\text { AUTHOR(S) - D.J.CARUTHERS }\end{array}$ & $10 / 09 / 74$ \\
\hline $\begin{array}{l}1208 \\
\text { (REV.) }\end{array}$ & $\begin{array}{l}\text { LASER STRIPPING FOR HIRE GREAKAGE OIAGMOSIS } \\
\text { AUTHORISI - R.T.HORN }\end{array}$ & $03 / 13 / 75$ \\
\hline $\begin{array}{l}1212 \\
\text { (REV.) }\end{array}$ & $\begin{array}{l}\text { MOLDING MINIATURE PLASTIC PARTS } \\
\text { AUTHORISI - D.P.POPE }\end{array}$ & $11 / 06 / 74$ \\
\hline 1213 & $\begin{array}{l}\text { APPLICATION OF MOIRE TECHNI QUES TO FIXTURELESS GAGING } \\
\text { AUTHORISI - C.H.BERRY, JR.. T.A.EPPES }\end{array}$ & $03 / 14 / 75$ \\
\hline 1214 & $\begin{array}{l}\text { APT/IGS NUMERICAL CONTROL SYSTEM } \\
\text { AUTHOR(S) - VOE.GROSS }\end{array}$ & $03 / 13 / 75$ \\
\hline $\begin{array}{l}1216 \\
\text { (REV.। }\end{array}$ & $\begin{array}{l}\text { DESIGN MANUAL FOR POTTING ELECTRONIC ASSEMALIES } \\
\text { AUTHORISI -6.0.SWANSON }\end{array}$ & $09 / 10 / 76$ \\
\hline 1223 & $\begin{array}{l}\text { SAMPLE SIZE TO SET SPECIFICATION LIMITS } \\
\text { AUTHOR SI - R.L.KIRKPATRICK }\end{array}$ & $02 / 12 / 75$ \\
\hline 1227 & $\begin{array}{l}\text { THE FEASIBILITY OF ELECTROPLATING PLATINUM-IRIDIUA OUT OF FUSED CHLORIDES } \\
\text { AUTHORISI - W.B.HARDING }\end{array}$ & $08 / 06 / 76$ \\
\hline $\begin{array}{l}1234 \\
\text { (REV.) }\end{array}$ & $\begin{array}{l}\text { CDAT25 TRANSFORMER LEAD SOLOER JOINT EVALUATION } \\
\text { AUTHOR(SI - P.F.BURK }\end{array}$ & $07 / 22 / 75$ \\
\hline 1235 & $\begin{array}{l}\text { MAINTENANCE MORK STANDARDS ANO COMPUTERILEO WORK SYSTEM } \\
\text { AUTHOR(S) - D.W.HUDGINS }\end{array}$ & $09 / 26 / 74$ \\
\hline 1239 & $\begin{array}{l}\text { DESIGN IMPRDVEMENTS FOR CYCLIC INJECTION MOLOS } \\
\text { AUTHORISI - M.J.BRONSON, D.C.ERUEGGE }\end{array}$ & $05 / 23 / 75$ \\
\hline $\begin{array}{l}1240 \\
\text { (REV.) }\end{array}$ & $\begin{array}{l}\text { COMPATIBILITY OF CONTAMINANTS AND PLASTICS MITH SOLVENTS } \\
\text { AUTHORISI - L.C.JACKSON }\end{array}$ & $07 / 22 / 75$ \\
\hline 1242 & $\begin{array}{l}\text { CALIBRATION METMOD FOR COORDIMATE MEASUR ING MACHINES } \\
\text { AUTHORISI - W.G.EBY }\end{array}$ & $07 / 22 / 75$ \\
\hline $\begin{array}{l}1244 \\
\text { (REV.) }\end{array}$ & $\begin{array}{l}\text { THREAD CERTIFICATION } \\
\text { AUTHORISI - C.H.BERRY, JR. }\end{array}$ & $11 / 06 / 75$ \\
\hline $\begin{array}{l}1245 \\
\text { (REV.) }\end{array}$ & $\begin{array}{l}\text { ACCUAACY OF MEIGHT METHOD USED TO DETERMINE ALUMINUM FOIL THICKMESS } \\
\text { AUTHORISI - M.C.LIPPERT }\end{array}$ & $08 / 11 / 76$ \\
\hline 1246 & $\begin{array}{l}\text { INFR ARED BAKING OF POSITIVE PHOTORES IST FOA HYBRIO MICROCIRCUIT FABRICATION } \\
\text { AUTHORISI - L.E.SCHANTZ }\end{array}$ & $08 / 13 / 76$ \\
\hline 1248 & $\begin{array}{l}\text { BUARS PRODUCED BY DRILLING } \\
\text { AUTHORISI - L.K.GILLESPIE }\end{array}$ & $08 / 11 / 76$ \\
\hline $\begin{array}{l}1252 \\
\text { (REV.) }\end{array}$ & $\begin{array}{l}\text { DEVELOPMENT OF AN IMPROVED BONDING FIXTUAE } \\
\text { AUTHORISI - J.D.SHEELEY }\end{array}$ & $01 / 30 / 76$ \\
\hline $\begin{array}{l}\text { 1254 } \\
\text { (REV.) }\end{array}$ & $\begin{array}{l}\text { PLATED THROUGH-HOLES IN FLEXCIRCUITS } \\
\text { AUTHOR(S) - R.A.HILE }\end{array}$ & $07 / 22 / 75$ \\
\hline
\end{tabular}


1257 HYBRID MICROCIRCUIT INTRACONNECTION PROCESSES

$08 / 11 / 74$ AUTHOR(S) - H.B.BONHAM

1260 EVALUATION OF POL YPHENYLENE SULFIDE

$05 / 12 / 75$

(REV.) AUTHORISI - C.L.WALTER

1264 LAPPING EQUIPMENT INSTRUMENTATIOA

$07105 / 77$ AUTHOR(S) - J.L.GRAY

1267 LDW FREQUENCY SHIELDING OF AN EXPERIMEMTAL MULTIHIRE CABLE

$01 / 30 / 76$

(REV.) AUTHOR(S) - D.H.STONE

1275 BEAM LEAD DEVICE TEMPERATURE TESTING

$08 / 02 / 76$ AUTHOR (S) - L.W.BROWM

1281 INPROVED PRECISION ALUMINUM ETCHANT

IREV.) AUTHOR(S) - H.H.KNOPF

1282 DUAL CHANNEL MIXER
AUTHORISI - J.H.CABLE

$00 / 13 / 76$

1284 an EVALUATION OF THE X-RAY FLUORESCENCE METHOD OF PRECIOUS METAL PLATING THICKNESS MEASUREMENTS

AUTHORIS) - J.S.CARSON, N.K.HEARN, C.B.PETTIE

1285 CAPPING FIXTURE FOR DISCRETE SEMICONDUCTORS AUTHOR (S) - M.C.COUPLAND

1286 PHOTOTOOL FABRICATION METHODS AUTHOR (S) - M.L.MILSOM

1288 PHOTORESIST APPLICATION BY ROLLER COATING AUTHORISI - L.E.SCHANTZ

$08 / 06 / 76$

$10 / 20 / 75$

1293 WELDING OF HERMETIC CONNECTORS
(REV.) AUTHOR(S) - D.E.HIEBER

$10 / 30 / 75$

$11 / 06 / 75$

$08 / 06 / 76$

1295 MONITORING SINUSOIDAL VIERATION INVIROMMENTS WITH A TV SYSTEM AUTHORISI - J.H.DAVIS

1297 AN AUTOMATIC RF-NETHORK ANALYZER AS A SUPPORT TOOL FOR ENGINEERING AND MANUFACTUR ING

AUTHOR SSI - J.L.TAYLOR

1298 FEAS IBILITY STUDY OF PROOUCTION OF 0.24 G/CM3-DENSITY POLYSTYRENE BEAD FOAM

(REV.) AUTHOR(S) - R.C.SWOBODA

1301 EVALUATION OF TPX MOLOING RESIN

IREV.) AUTHORISI - C.L.MALTER

1303 BURRS PRODUCEO BY SIDE-MILLING CUTTERS

AUTHOR(S) - L.K.GILLESPIE

(REV.)

1315 REWORK OF MULTILAYER PRINTEONIR INGQOARD ASSEMOLIES AUTHORISI - O.H.CLEMENTS

$08 / 02 / 76$

$07 / 05 / 77$

$08 / 13 / 76$

$08 / 13 / 76$

$10 / 20 / 75$

$11 / 21 / 75$

$08 / 02 / 76$

1316 FRICTION ANDMEAR STUOIES FOR BONDED THIMFILM LUBRICANTS O8/O6/76 AUTHOR (S) - D.P.POPE

II/66775

1319

A PRESS FIT INTERFERENCE-LIMTT STOOT FG MINTATOKE ASSERLIES

$05 / 12 / 75$

1325 SORTING AND HANOLING LASER TARGETS

AUTHOR(S) - J.H.KUEHWL

$01 / 30 / 76$

1345

FLEXIBLE POL YURETMANE FOAM

1346 INTERCONNECTION TECHNOLOGY

$08 / 11 / 76$ AUTHORISI - H.P.BECK, J.R.SIM

1349 SANOIA/BEMDIX STANDARD PROCESS CAPABILITY STUOY OF PRINTED HIRING BOAROS AUTHOR(S) - J.L.MILSON

$08 / 11 / 76$

13.53 MEASURING PENETRATION DEPTH OF ELECTROM CEAM WELDS

$08 / 14 / 75$ AUTHOR(S) - J.H.MILL

1354 PROCESS ACCEPTANCE AND ADJUSTMENT TECHMIQUES FOR SWISS AUTOMATIC SCREN 


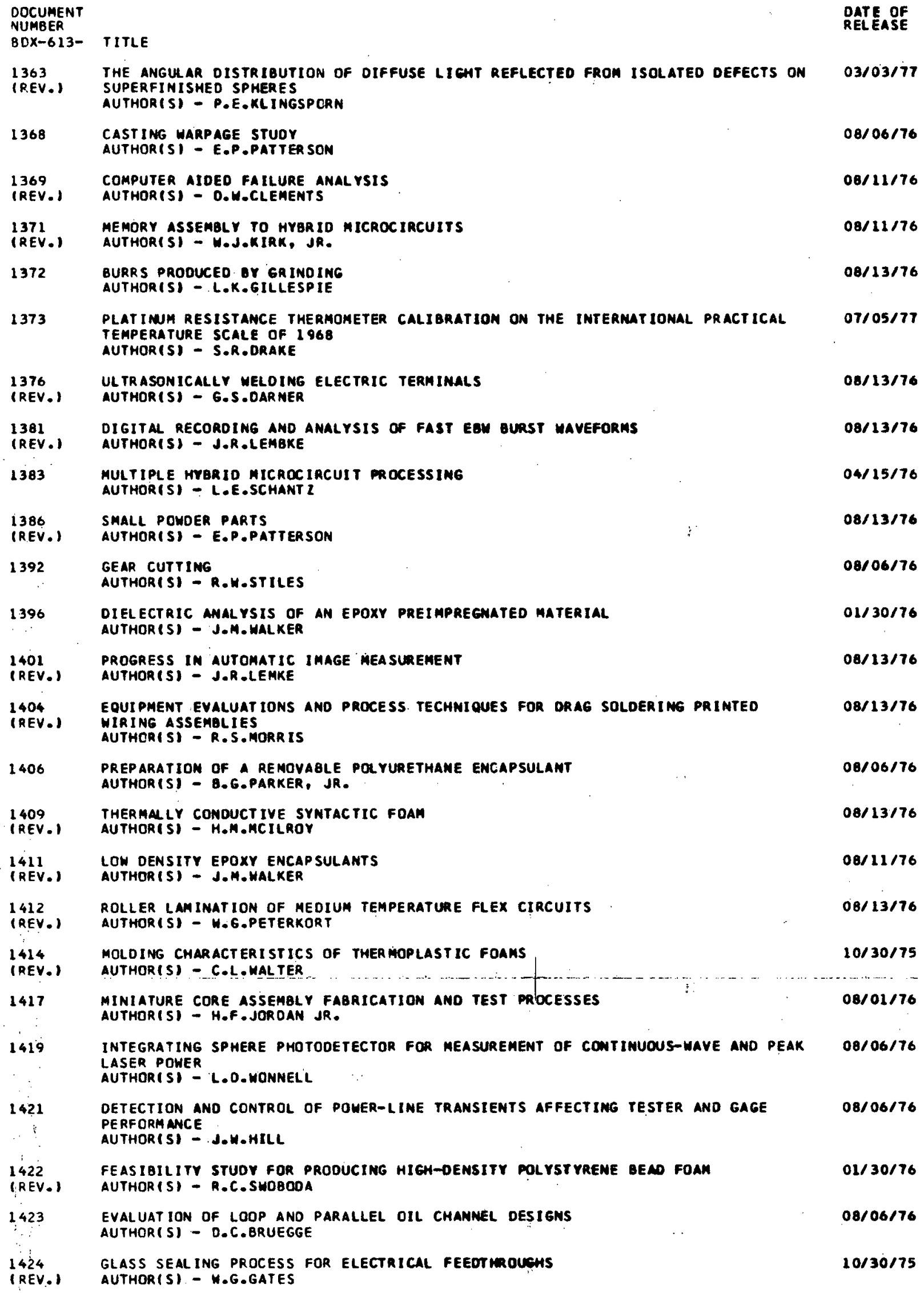


BDX-613- TITLE

1425 INVESTIGATION OF NEM ENGINEERING THERMOPLASTICS

(REV.) AUTHOR(S) - C.L.MALTER

1429 SKIVING

AUTHOR(S) - L.K.GILLESPIE

1434 ELECTRICAL CONOUCTIVITY STUOY OF ETCHED-OUT PLATED-THROUEH MOLES IN DOUBLE-SIOED PRINTED WIRING BOARDS AUTHORISI - R.J.SETTER

1435

EVALUATION OF HIGH DENSITY BRAID TERMIMATION FATIGUE LIFE AND CONDITION AUTHOR(S) - E. BELAROE

1.443 HAND OEBURR ING OF PRECISION MINIATURE PARTS AUTHORISI - L.K.GILLESPIE

1453 INDUCTION SOLDERING EVALUAT ION AUTHOR(S) - E.R.FRIEBE

1454 SURFACE PREPARATION OF KAPTON FOR ALUMIMUM DEPOSITION (REV.) AUTHOR(S) - M.G.ULITCHNY

1456

FAILURE MODES OF BEAM LEAD SEMICONOUCTORS IN THIN FILM HYBRIO MICROCIRCUITS (CONFERENCE PAPER)

AUTHOR (S) - J.H.SHAFFORD

HAND PUNCHING HOLES IN UNFIRED 99.5 PERCEMT ALUMIMA SUBSTRATES

AUTHOR(S) - P.L.BLESSNER

1462 FACE-UP BONDING OF BEAM LEAO DEVICES

(REV.) AUTHOR(S) - A.0.BENDURE

1464 AUTOMATIC LEAD FRAME PLATING

AUTHOR(S) - D.M.JARBOE

1465 FEASIBILITY STUOY OF DIODE PRECONDITIONING

(REV.) AUTHOR(S) - J.P.GRACEY

1466

(REV.)

QUANTITATIVE AUGER ELECTRON ANALYSIS OF GOLD-COPPER ALLOYS

AUTHOR(S) - R.L.HANLIN, D.L.STOLTZ

1468 SURFACE EFFECTS ON SEMICONDUCTORS

AUTHOR(S) - E.L.RAUCH

1469 EXPOSURE STUOY FOR DRY-FILM PHOTORESISTS

AUTHOR(SI - M.L.WILSON

147

THE NECESSITY OF CMARACTERI IING HYBRIO MICROCIRCUIT ELENENTS AT MICRONAVE FREQUENCIES

AUTHOR(S) - G.D.O.OELL

1478

GROUP TECHNOLOGY

AUTHORISI - C.P.ROME

1479 SOLIO STATE CONNECTOR

AUTHORISI - R.A.HAYES

1480 SYNTHES IS OF POLYAMINOB ISMALEMI OE

AUTHOR (S) - D.R.JAMIESON

1482 FIXTURING AND HANDL ING OF MINIATURE COMPONENTS

AUTHORISI - L.K.GILLESPIE

1485 INJECTION MOLDING THERMOPLASTIC RUBBER

(REV.) AUTHOR(S) - J.R.PORTER

148

DESIGN MODIFICATION FOR HIGH VOLTAGE ELECTRON BEAM MELDING MACHINE AUTHOR(S) - B.O.FR ICKE

1489. HYDROLYTIC STABILITY OF ADIPRENE L-100 AND ALTERNATE MATERIALS AUTHOR (S) - D.J.CARUTHERS

1491 ANALYSIS OF THICK FILA CONDUCTOR IHKS AUTHOR (S) - D.H.BREHER

1493

DEVELDPMENT OF AN AUTOMATED ENCAPSULATION SYSTEM AUTHOR(S) - J.B.GALLAHER

1496 (REV.)

MATERIAL AND PROCESSING COMPARI SONS AUTHOR $(S)$ - D.J.CARUTHERS
DATE OF

RELEASE

$08 / 13 / 76$

$08 / 11 / 76$

$01 / 30 / 76$

$09 / 09 / 76$

$11 / 21 / 75$

$08 / 11 / 76$

$10 / 30 / 75$

$08 / 13 / 76$

$08 / 11 / 76$

$09 / 10 / 76$

$11 / 21 / 75$

$09 / 10 / 76$

$01 / 30 / 76$

$01 / 30 / 76$

$09 / 10 / 76$

$09 / 09 / 76$

$04 / 15 / 70$

$03 / 15 / 77$

$07 / 05 / 77$

$09 / 10 / 76$

$09 / 09 / 76$

$09 / 10 / 76$

$09 / 09 / 76$

$09 / 10 / 76$

$08 / 29 / 77$

$03 / 15 / 77$ 

OPTICAL SYSTEM FOR AUTOMATEO INSPECTION OF HIGHLY REFLECTIVE SPHERICAL SURF ACES AUTHOR(S) - P.E.KLINGSPORN

\begin{tabular}{|c|c|c|}
\hline $\begin{array}{l}1500 \\
\text { (REV.) }\end{array}$ & $\begin{array}{l}\text { COVER EVAL UATION FOR LARGE HYBR IO MICAOCIRCUITS } \\
\text { AUTHOR(S) - J.H.WILLIAMS }\end{array}$ & $09 / 24 / 76$ \\
\hline 1502 & $\begin{array}{l}\text { EFFECTS OF DRILLING VARIABLES ON BURR PROPERTIES } \\
\text { AUTHORISI - L.K.GILLESPIE }\end{array}$ & $09 / 24 / 76$ \\
\hline 1503 & $\begin{array}{l}\text { BURRS PROOUCEO BY ENO MILLING } \\
\text { AUTHORISI - L.K.GILLESPIE }\end{array}$ & $10 / 01 / 76$ \\
\hline $\begin{array}{l}1507 \\
\text { (REV.) }\end{array}$ & $\begin{array}{l}\text { SILICONE ELASTOMER EVALUATION FOR CABLE JACXET APPLICATION } \\
\text { AUTHOR(S) - R.R.CROCKETT }\end{array}$ & $09 / 24 / 76$ \\
\hline $\begin{array}{l}1512 \\
\text { (REV.) }\end{array}$ & $\begin{array}{l}\text { ECONOMIC OPTIMIZATION TECH IQUES APPLICABLE TO PRECISION MINIATURE MACHINING } \\
\text { AUTHOR(S) - L.K.GILLESPIE }\end{array}$ & $09 / 24 / 76$ \\
\hline 1519 & $\begin{array}{l}\text { TEMPERATURE OEP ENDENT PROPERTIES OF POTTIMG MATERIAL } \\
\text { AUTHOR(S) - J.M.WALKER }\end{array}$ & $07 / 05 / 77$ \\
\hline $1520 *$ & $\begin{array}{l}\text { FLOW ANO EXHAUST SYSTEM FOR CHEMICAL YAPOR DEPOSITION OF TUNGSTEN-RHENIUH } \\
\text { ALLOY } \\
\text { AUTHOR(SI - C.E.BUSHEY }\end{array}$ & $09 / 10 / 76$ \\
\hline 1.522 & $\begin{array}{l}\text { REMOVAL OF SILICONE GREASE ANO OIL CONTAMINANTS } \\
\text { AUTHORISI - L.C.JACKSON }\end{array}$ & $03 / 22 / 76$ \\
\hline 1523 & $\begin{array}{l}\text { EVALUATION OF ALKAL INE ETCHANTS FOR PRINTEO MIRIMG BOAROS } \\
\text { AUTHORISI - M.E.EVANS }\end{array}$ & $09 / 24 / 76$ \\
\hline 1524 & $\begin{array}{l}\text { WIGGLY PMASE SHIFTERS ANO DIRECTIOMAL COLPLERS FOR RADIO FREQUENCY HYBRID } \\
\text { MICROCIRCUIT APPLICATIONS (CONERENCE PAPERI } \\
\text { AUTHOR(S) - D.D.PRIGEL. J.L.TAYLOR }\end{array}$ & $08 / 13 / 76$ \\
\hline 1530 & $\begin{array}{l}\text { DEPOTTING ELECTRONIC ASSEMBLIES } \\
\text { AUTHORISI - C.J.CAMP }\end{array}$ & $07 / 05 / 77$ \\
\hline $\begin{array}{l}1531 \\
\text { (REV.I }\end{array}$ & $\begin{array}{l}\text { FRACTUAE MECHANICS TESTING CAPABILITY } \\
\text { AUTHORISI - M.G.ULITCHNY }\end{array}$ & $09 / 09 / 76$ \\
\hline 1532 & $\begin{array}{l}\text { MANUFACTURING PROCESSES FOR, HYBRID MICROCIRCUITS CONTAINING VIAS (CONFERENCE } \\
\text { PAPER) } \\
\text { AUTHOR(S) - P.L.BLESSNER, A. LAUDEL, AR., D.P.NORWOOD }\end{array}$ & $08 / 13 / 76$ \\
\hline 1533 & $\begin{array}{l}\text { CLOSED CIRCUIT TELEVISION MELOING ALIGNMENT SYSTEM } \\
\text { AUTHOR(S) - G.S.DARNER }\end{array}$ & $09 / 24 / 76$ \\
\hline 1534 & $\begin{array}{l}\text { HIGH TEMPERATURE SYNTACTIC FOAM } \\
\text { AUTHOR(S) - H.M.MCILROY }\end{array}$ & $07 / 05 / 77$ \\
\hline 1535 & $\begin{array}{l}\text { PRODUCTION COATING OF VIAS IN ALUMINA SUBSTRATES WITH VACUUM EVAPORATED } \\
\text { CHROMIUM ANO GOLD (CONFERENCE PAPER) } \\
\text { AUTHORISI - J.A.LOSURE }\end{array}$ & $08 / 13 / 76$ \\
\hline 1536 & $\begin{array}{l}\text { HANOL ING AUTOMATION AND TEMPERATURE CONTROL ADOED TO TRANSISTOR S- PARAMETER } \\
\text { MEASUREMEMT (CONFERENGE PAPER) } \\
\text { AUTHOR(S) - R.E-MARKLEY }\end{array}$ & $08 / 13 / 76$ \\
\hline 1542 & $\begin{array}{l}\text { TRANSFER MOLOING DF CLOSE-TOLERANCE PARTS } \\
\text { AUTHOR }(S)=\text { B.A.HEFNER }\end{array}$ & $08 / 29 / 77$ \\
\hline 1546 & $\begin{array}{l}\text { EXTRUDE HONE DEBURR ING WITH X-BASE MEDIA } \\
\text { AUTHOR(S) - L.K.GILLESPIE. }\end{array}$ & $09 / 09 / 76$ \\
\hline $\begin{array}{l}1549 \\
\text { (REV.) }\end{array}$ & $\begin{array}{l}\text { PREPARATION OF URETHANE ADHESIVES } \\
\text { AUTHORISI - B.G.PARKER, JR. }\end{array}$ & $09 / 24 / 76$ \\
\hline $\begin{array}{l}1552 \\
\text { (REV.) }\end{array}$ & $\begin{array}{l}\text { COMPUTER AIOED FAILURE ANALYSIS } \\
\text { AUTHORISI }- \text { R.S.SMITH }\end{array}$ & $09 / 24 / 76$ \\
\hline 1557 & $\begin{array}{l}\text { LOW TEMPERATURE ALUMINUM SOLDERING AMALYSIS } \\
\text { AUTHORISI - M.6.PETERKORT }\end{array}$ & $10 / 01 / 76$ \\
\hline 1559 & $\begin{array}{l}\text { DEBURRING OY CENTRIFUGAL BARREL TUMBLING } \\
\text { AUTHORISI - L.R.GILLESPIE }\end{array}$ & $09 / 22 / 76$ \\
\hline 1563 & LASER CONTOURED AND DRILLEO SUBSTRATES FOR THICK FILA APPLICATIONS & $09 / 24 / 76$ \\
\hline
\end{tabular}

DATE OF Release

$09 / 22 / 76$ $09 / 24 / 76$

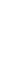




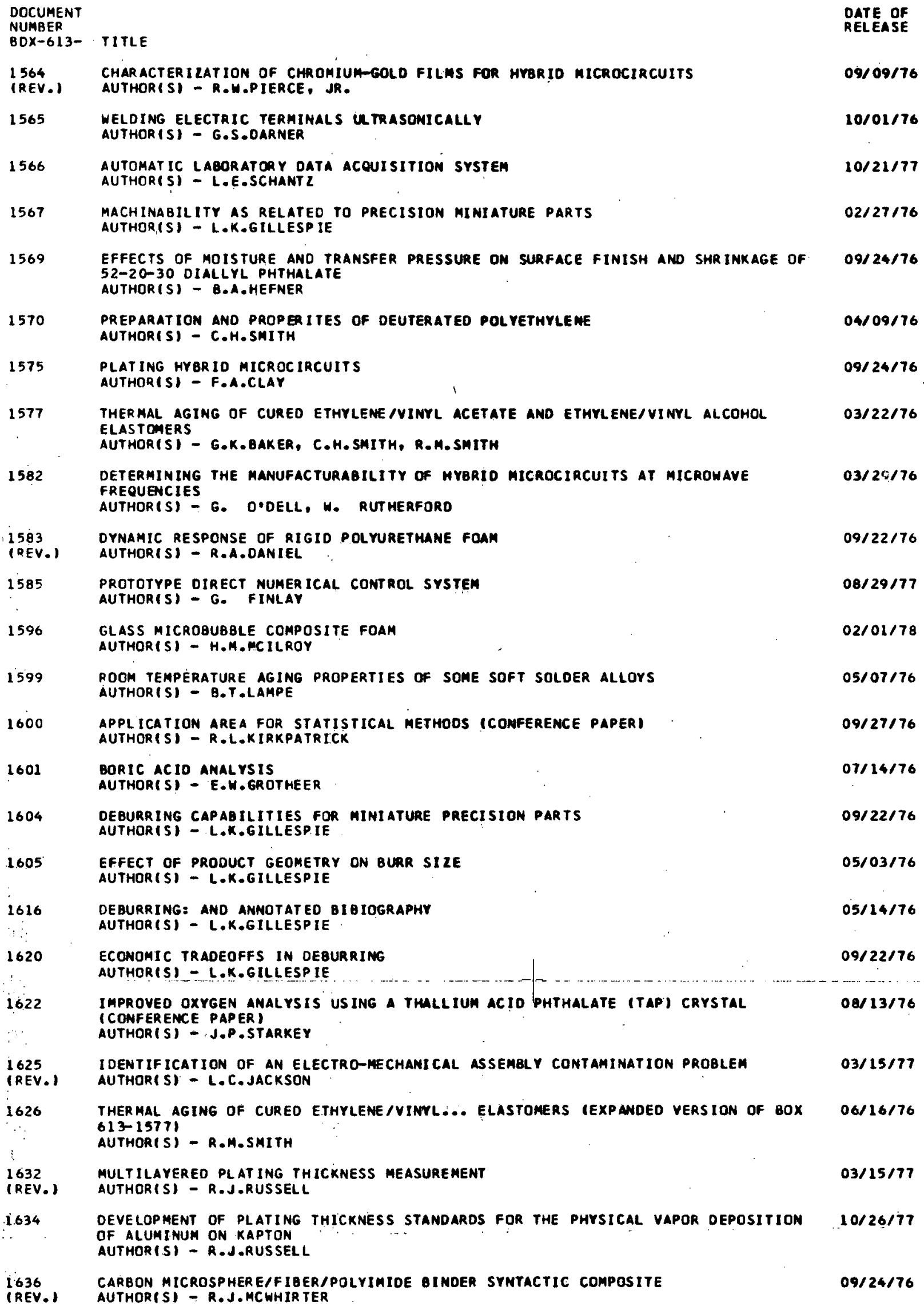

1599 ROOM TEMPERATURE AGING PROPERTIES OF SOME SOFT SOLOER ALLOYS 05/07/76

1604 DeburRING CaPABILITIES For mintatuRe PRECISION PARTS $09 / 22 / 76$

1626 THER HAL AGING OF CURED ETHYLENE/VIMYL... ELASTOMERS (EXPANDEO VERSION DF BOX O6/16/76

$09 / 09 / 76$

$10 / 01 / 76$

$10 / 21 / 77$

$02 / 27 / 76$

$09 / 24 / 76$

$04 / 09 / 76$

$09 / 24 / 76$

$03 / 22 / 76$

$09 / 22 / 76$

$08 / 29 / 77$

$02 / 01 / 78$

$09 / 27 / 76$

$07 / 14 / 76$

$05 / 03 / 76$

051476

$09 / 22 / 76$

$08 / 13 / 76$

$03 / 15 / 77$

$03 / 15 / 77$

$10 / 26 / 77$

$09 / 24 / 76$ 

AUTHOR(S) - L.A. WOLFE

1651 MINIATURE INTERCONNECTION DEVELOPMENT

(REV.) AUTHOR(S) - O.L.HOLLAR

1652 A MOVING HEAO DISC OPERATING SYSTEN FOR AN 8542A AUTOMATIC METWORK ANALVZER (CONFERENCE PAPER)

AUTHORISI - M.D.JACKSON, R.M.TELLEZ

1655 STRESS REL IEF TECHMIQUES FOR REOUCING THERMAL FATIGUE IN SOLDER JOINTS AUTHORISI - D.M.JARBOE

1656 CURE ANALYSIS OF AN ADHESIVE PRIMER AUTHOR S S - J.M.HUBACH

1657 FABRICATION OF MULTILAYER PRINTED MIRING BOARDS AUTHOR(S) - J.L.WILSON

1666 BRAZING TITAMIUM STRUCTURES

(REV.) AUTHOR(S) - H.B.PRESSLY 1668 DIGITAL ANALYSIS OF EBW BUR ST WAVEFORMS
AUTHOR(S) - JOR.LEMBKE

1673 FACTORS AFFECTING VISUAL IMSPECTION OF THIN FILM METWOAKS

$11 / 29 / 77$

$07 / 05 / 77$

$02 / 06 / 78$

$03 / 15 / 77$

$02 / 06 / 78$

$02 / 06 / 78$

1603 CERAMIC-TO-METAL SEALING

$03 / 15 / 77$

(REV.) AUTHOR (S) - M.G.GATES

1684

WEAR PREVENTION IN MOLDS USEO TO MOLD BORON-FILLED ELASTOMERS ICONFERENCE PAPER I AUTHOR(S) - R.S.PLUMO

1685 SHIELDING EFFECTIVENESS OF SINGLE CONOUCTOR FLAT SHIELDEO CABLES AUTHOR(S) - G.S.DARNER

1688 EOITING CAPABILITY FOR DIRECT NUMERICAL CONTROL AUTHOR(S) - G.L.FINLAY

1691 DEVELOPMENT OF AN AUTOMATED FOAM PROCESSING SYSTEN AUTHOR(S) - J.B.GALLAHER

$10 / 24 / 77$ AUTHOR(S) - R.D.MAGNER

1695 CONTAMINANT CLEANING FOR CRITICAL ELECTRICAL ASSEMBLY AREAS AUTHORISI - L.C.JACKSON

$02 / 06 / 78$ AUTHOR(S) - L.K.GILLESPIE

ANNTHTLATTE THE BURA …. AUTHOR $(S)$ - L.K.GILLESPIE

1706 SOLDERABILITY TEST DEVELOPMENT AUTHOR(S) - D.M.JARBOE AUTHOR(S) - R.d.RUSSELL

$02 / 06 / 78$ DETERMINATION DF GOLOMCOD
ANALYSIS
AUTHOR(S) - D.M.STARKS

MULTIPURPOSE LABORATORY TEST SYSTEM APPLYING -CAMC STANDAROS ICONFERENCE PAPERI AUTHOR(S) - J.L.BOWERS 


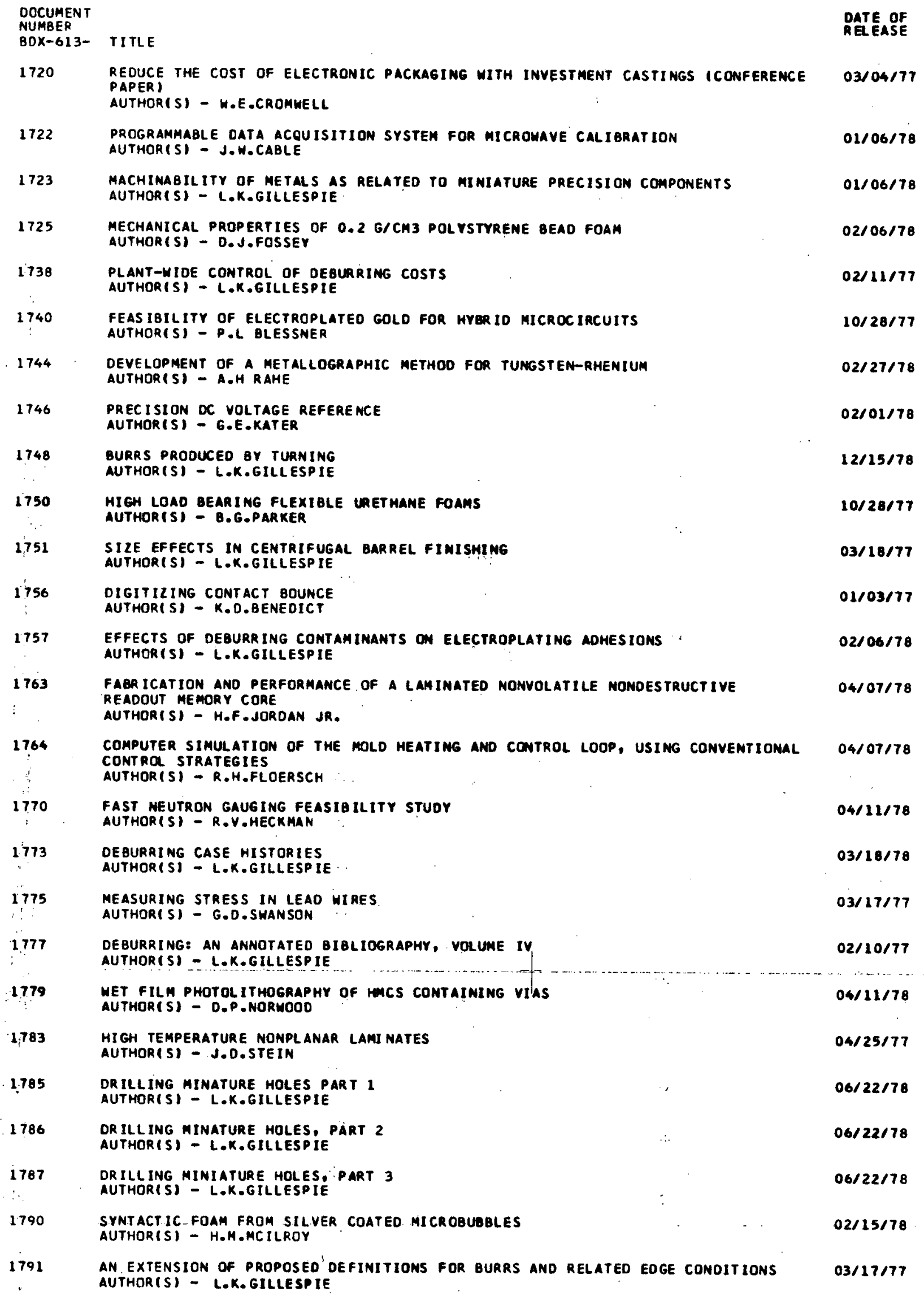


1793 THE BURR: A 1977 REPOAT ON THE TECHWLLOG OF REDUCIMG ITS COS AUTHOR(S) - L.K.GILLESPIE

1795

multilayereo PLATING THICKNESS AUTHOR(S) - R.J.RUSSELL

1796 PREPARATION AND PROPERTIES OF BLOCKED DIISOCYANATES AUTHOR(SI - B.G.PARKER

$03 / 18 / 77$

$1800 \quad$ HMC-TO-FLATPACK ATTACHMENT
AUTHOR(S) - L.R. ZAWICKI

$08 / 29 / 78$

1803 HMC SPRAY, DEVELOPING, STRIPPING, ANO ETCHING
AUTHOR(S) - P.L.BLESSNER

1803 HMC SPRAY, DEVELOPING, STRIPPING, ANO ETCMING
AUTHOR(S) - P.L.BLESSNER

02122178

$04 / 11 / 78$

1805 PLATING THICKNESS STANDARDS OT/28/78

AUTHOR(SI - R.J.RUSSELL

$04 / 11 / 78$

SEMICONDUCTOR DIE ATTACHAENT

$04 / 11 / 78$

TELEMETRY TEST METHODS

AUTHOR(S) - J.L.BOWERS

1812 MECHANICAL ANO MICROSTRUCTURAL PROPERTIES CMARACTERIzATION OF HEART-TREATEO BETA EXTRUDED TI-6AL-6V-2SN AUTHORISI - M. G.ULITCHNY

IBI3 SEMIAUTOMATIC VISUAL INSPECTION SYSTEM FOR THIN-FILM METWORKS AUTHOR(S) - R.E.HINES

1816 CHEMICAL AND PHYSICAL CHARACTERILATION OF A KEVLAR-FILLED EPOXY MOLOING COMP OUND

AUTHOR(S) - J.H.MCFARLAND

:1823 DENSITY MEASUREMENTS OF CURVED FOAM PARTS USIMG RADIOGRAPHIC-FILA TECHNIQUE AUTHOR(S) - H.J.BOWDEN

$04 / 04 / 77$

$10 / 20 / 78$

$04 / 11 / 78$

$12 / 15 / 78$

$X$-RAY POLE ANALYSIS

AUTHOR (S) - D.M.JAR BOE

$10 / 20 / 78$

material EVALUATION OF POLYURETHANE FoAM, 0.05 G/CM3 DENSITY

AUTHOR (S) - J.R.FENDER

$04 / 11 / 78$

EOUIPMENT FOR A LASER SURFACE MEASUR ING SYSTEM

$12 / 15 / 78$

AUTHOR (S) - R.T. STAGNER.

$08 / 29 / 78$

1853 CHEMICAL. THERMAL, AND OYNAMIC MECHANICAL PROPERTIES OF FIVE aDHESIVES AUTHOR(S) - G.K.BAKER

1857 CHARACTERIZATION OF THIN FILM MICROSTRIP ATTENUATORS AT MICROHAVE FREQUENCIES

1 AUTHORISI - F.R.SHITH

$12 / 15 / 78$

I 865 SOLDERING AUTOHATION

AUTHOR $(S)=$ S. LANDERS

i1/15/78

THE TIAE-TEMPER ATURE CORRESPONDENCE OF THE DYNAMIC MEChanICAL PROPERTIES OF HALTHANE

AUTHORISI - G.K.BAKER

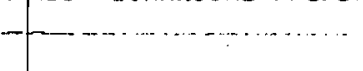

$11 / 30 / 78$

1867

1868

QUANTITATIVE COMPARISON. OF ELECTRONIC COMPONENT-SOLOER JOINT STRESS RELIEF IN ENCAPSULATED ASSEMBLIES AUTHOR(S) - D.A.CUMMINGS

1870 SIMULATION MODELING OF AN AUTOMATED MATERIAL STORAGE/RETRIEVAL SYSTEM AUTHOR(S) - J. GALLEGOS

$11 / 30 / 78$

1873 DEPOSITION ANO CHARACTERIzATION OF VACUUM-OEPOSITEO ALUMINUM FILM ON KAPTON LAMINATES AUTHORISI - D.M.SHERMAN

1877 LASER CUTTING OF KEVLAR LAMINATES AUTHORISI - R. A.VANCLEVE

$02 / 27 / 78$

$07 / 23 / 78$

$10 / 21 / 77$

1880 - -.SHELF LIFE AGING OF DC-302-SILICONE HOLOING COMPOUMD AUTHORISI - H.H.MCFARLAND

$-08 / 29 / 78$

$08 / 29 / 78$

1887 PROCESS EFFECTS ON HIGH VOLTAGE ELECTRICAL ASSEMBLIES AUTHOR (S) - D.A.CUMMINGS 
1888 CANADA'S 100 MILLION DOLLAR THORNIN CAMADIAN MACHIMERY ANO METALMOAKIMG AUTHOR(SI - L.K. GILLESPIE

$08 / 05 / 77$

1890 CHARACTERILATION OF LOW DENSITY RIGIO URETHAME FOAM

$11 / 15 / 78$ AUTHORISI - F.M.LARSEN

1891 NEEDED RESEARCH ON BURRS, DEBURRING, AND EOGE FIMISHING ITO BE PUBLISHED BY SMEI AUTHOR(S) - L.K.GILLESPIE

1903 DEVELOPMENT OF BETA BACKSCATTER PROBES USIMG HIGH-INTEMSITY SOURCES (CONFERENCE PAPER) AUTHOR(S) - R.V.HECKMAN

1905

INVESTIGATION OF METHYLPENTENE POLYMER (TPX) MOLD.IMG RESIN AUTHOR(S) - C.L.MALTER

1907 UNIVERSAL MONITOR FOR CO2 LASERS (COMFEREMCE PAPER) AUTHOR(S) - L.D.WONNELL

$04 / 11 / 78$

$11 / 29 / 77$

$11 / 15 / 78$

$12 / 19 / 78$

$11 / 29 / 77$

$05 / 15 / 78$

$08 / 29 / 78$

$12 / 15 / 78$

$12 / 15 / 78$

$07 / 28 / 78$

$12 / 15 / 78$

$11 / 15 / 78$

$12 / 29 / 77$

$10 / 20 / 78$

$11 / 29 / 77$ AUTHOR STI-E.H.MORR IS

1950

CONTROL SYSTEM MODELING OF A CENTRIFUGE (CONFERENCE PAPERI

$11 / 29 / 77$ AUTHOR(S) - J.C.VAJDIC

REHABILITY OF PACKAGED BEAM-LEAO TRANSISTORS BUILT HITH OIFFERENT PRE-SEAL

$10 / 20 / 78$ ASSEMBLY TECHNIQUES AUTHORISI - J.P.GRACEY AUTHORISI - R.S.HEDGES

$05 / 15 / 78$ AUTHORISI - J.H.STEELE

1964 POLYURETHANE FOAM $0.056 /$ CM3 DENSITY

$11 / 07 / 78$ AUTHORIST - J.R.FENOER 


\begin{tabular}{|c|c|c|}
\hline $\begin{array}{l}\text { DOCUMENT } \\
\text { NUMBER }\end{array}$ & & $\begin{array}{l}\text { OATE DF } \\
\text { REEASE }\end{array}$ \\
\hline $80 x-613-$ & TITLE & \\
\hline 1967 & $\begin{array}{l}\text { CONTACTOR SPEEDS S-PARAMETER TESTS } \\
\text { AUTHOR(SI - M.W.HOGAN, R.E.MARKLEY }\end{array}$ & $01 / 17 / 78$ \\
\hline 1968 & $\begin{array}{l}\text { MASS PARALLEL INTERFACE INPUTS DATA TO PDP-11 } \\
\text { AUTHOR(S) - M. M.HOGAN }\end{array}$ & $01 / 17 / 78$ \\
\hline 1969 & $\begin{array}{l}\text { OPTIMILATION OF LEAD FRAME BOND PARAMETEAS FOR PROOUCTION OF RELIAGLE } \\
\text { THERMOCOMPRESSION BONDS } \\
\text { AUTHORISI - R.J.BLALER, W.A.PIPER }\end{array}$ & $01 / 27 / 78$ \\
\hline 1977 & $\begin{array}{l}\text { ADVANCES IN DEBURRING } \\
\text { AUTHOR(S) - L.K.GILLESPIE }\end{array}$ & $02 / 02 / 78$ \\
\hline 1979 & $\begin{array}{l}\text { MOLO HEATING AND COOLING SYSTEMS } \\
\text { AUTHOR }(S) \text { - R.H.FLOERSCH }\end{array}$ & $12 / 15 / 78$ \\
\hline 1980 & $\begin{array}{l}\text { DIGITAL CONTROLLER OESIGN, ANALYSIS, ANO IMPLEMENTATION FOR THE MOLO MEATIMG } \\
\text { AND COOLING SYSTEM } \\
\text { AUTHORISI - R.H.FLOERSCH }\end{array}$ & $12 / 15 / 78$ \\
\hline 1981 & $\begin{array}{l}\text { REMOVAL OF METHYLENE DIANILINE FROM CHEMICAL PLANT WASTEMATER } \\
\text { AUTHORISI - D.A.YOUNG }\end{array}$ & $03 / 23 / 78$ \\
\hline 1985 & $\begin{array}{l}\text { VIBRATORY DEBURRING: AN AMALYSIS OF COSTS (MACHINE TOOL AND BLUE BOOK) } \\
\text { AUTHORIS) - L.K.GILLESPIE }\end{array}$ & $02 / 10 / 78$ \\
\hline 1986 & $\begin{array}{l}\text { HAND DEBURR ING: A NECESSITY THAT CAM DE IMPROVED } \\
\text { AUTHORISI - L.K. GILLESPIE }\end{array}$ & $08 / 29 / 78$ \\
\hline 1987 & $\begin{array}{l}\text { ENVIRONMENTAL SUMMARY FOR CALENOAR YEAR } 2977 \\
\text { AUTHORISI - }\end{array}$ & $07 / 28 / 78$ \\
\hline 1.988 & $\begin{array}{l}\text { RESIN PROCESS CONTROLS } \\
\text { AUTHORISI - D.E.SHEPKERO }\end{array}$ & $11 / 07 / 78$ \\
\hline 1990 & $\begin{array}{l}\text { MOLO HEATING ANO COOLING SYSTEMS } \\
\text { AUTHORISI - R.H.FLOERSCH }\end{array}$ & $10 / 20 / 78$ \\
\hline 1993 & $\begin{array}{l}\text { DEPOSITION OF ALUMINUM-COPPER ALLOY ON LMIMATED POLYIMIDE SUBSTRATES FROM } \\
\text { AN RF INDUCTANCE SOURCE } \\
\text { AUTHOR(S) - G.J.HALE }\end{array}$ & $11 / 30 / 78$ \\
\hline 2003 & $\begin{array}{l}\text { A BOUNDARY VALUE DETERMINATION OF CONTACT AMGLE } \\
\text { AUTHOR(S) - J.D.EMERY }\end{array}$ & $03 / 17 / 78$ \\
\hline 2006 & $\begin{array}{l}\text { COMPUTER AIDED FAILURE ANAL YSIS } \\
\text { AUTHOR (S) - R.S.SMITH }\end{array}$ & $12 / 15 / 78$ \\
\hline 2010 & $\begin{array}{l}\text { DIGITAL CONTROLLER FOR CYCLIC TEMPERATUAE CONTROL } \\
\text { AUTHOR(S) - R.H.FLOERSCH }\end{array}$ & $07 / 28 / 70$ \\
\hline 2011 & $\begin{array}{l}\text { TESTING THE INTERLAMINATE AOHES IOM OF MULTILAYER PRINTED WIRING BOARDS } \\
\text { AUTHORISI - J.H.LULA }\end{array}$ & $10 / 20 / 78$ \\
\hline 2016 & $\begin{array}{l}\text { FRAUNHOFER OIFFRACTION PATTERNS FRON APERTURES ILLGMINATED WITH MOMPARALLEL } \\
\text { LIGHT } \\
\text { AUTHORIS) - P.E.KLINGSPORN }\end{array}$ & $03 / 23 / 78$ \\
\hline 2017 & 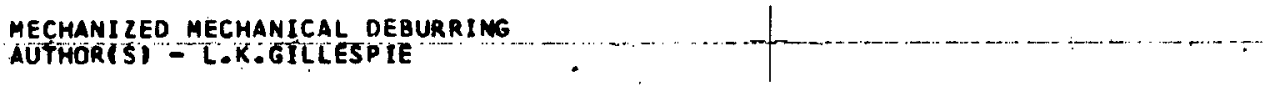 & $03 / 30 / 78$ \\
\hline 2018 & $\begin{array}{l}\text { DIRECT NUMERICAL CONTROL AT BENDIX KAMSAS CITY DIVISION } \\
\text { AUTHORISI - G.L.FINLAY }\end{array}$ & $05 / 11 / 78$ \\
\hline 2020 & $\begin{array}{l}\text { COMPUTER APPLICATIONS ANO HYBRID MICROCIACUITS } \\
\text { AUTHOR }(S)^{-}-\text {L. SHEETS }\end{array}$ & $03 / 30 / 78$ \\
\hline 20.22 & $\begin{array}{l}\text { LASER HELDING OF ELECTRICAL CONNECTIONS } \\
\text { AUTHOR(S) - F.R.BAUER }\end{array}$ & $12 / 15 / 78$ \\
\hline 2028 & $\begin{array}{l}\text { CONT AMINANT DETECTION CHARACTER IZATION, ANO REMOYAL BASED ON SOLUBILITY } \\
\text { PARAMETER PRINCIPLES } \\
\text { AUTHORISI - L.C.JACKSON }\end{array}$ & $05 / 10 / 78$ \\
\hline 2030 & $\begin{array}{l}\text { GAS PLASMA EFFECTS ON PRINTEO CIRCUIT COARO MATERIALS } \\
\text { AUTHORISI - L.C.JACKSON }\end{array}$ & $11 / 30 / 78$ \\
\hline 2031 & $\begin{array}{l}\text { IMPROVED PROCESS FOR WELOING STUDS TO RING AHO STUO ASSEMBLIES } \\
\text { AUTHOR (S) - G.P.MILLER }\end{array}$ & $12 / 15 / 78$ \\
\hline
\end{tabular}


2037 HIGH DENSITY BRAID CABLE SHIELDS

$12 / 15 / 70$ AUTHOR(S) - E. BELAROE

2038 RTV TOOLING EVALUATION AUTHORISI - E. BELARDE

$12 / 19 / 78$

2039 COOL ING ALUMINUM MOLOS USING HEAT PIPES

$11 / 30 / 70$ AUTHOR(S) - D.R.HAHM

$06 / 22178$

FINITE ELEMENT ANALYSIS OF LASER IMOUCEOTHERMAL SHOCK

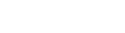

2045 OBSERYATIONS ON IMPLEMENTING GROUP TECHNOLOGY FOR PAECISION MINIATURE PARTS (JOURNAL ARTICLE UC-38) AUTHOR(S) - L.K.GILLESPIE

2051 MOORE NUMBER 5 DIAMONO TURNING MACHINE DESIGN ANO DESCRIPTION AUTHOR (S) - D.L.SETTER

2060 DEVELOPMENT OF A COMPARATIVE ANALYZER FOR MICROPROCESSOR AUTHOR (S) - E.S.ROLFE, ...

$06 / 22178$

2067 BARREL TUMBL ING IS IT ECONOMICAL? AUTHORIS) - L.K.GILLESPIE

2081 DESTRUCTIVE TESTING OF HYBRIO MICROCIRCUITS CONTAINING THERMOCOPRESSIOH BONDED OFYICES ANO LEAC-INDIUM SOLDERED CAPACITORS AUTHORISI - D.A.RATHEUN

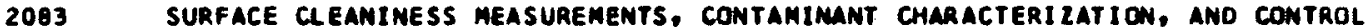
AUTHOR(S) - L.C.JACKSON

2100 HAND DEBURRING A PLAGUE OR THE MOST ECOMOAICAL MAYP AUTHOR(S) - L.K.GILLESPIE

210

THREE-BOOY ABRASIVE WEAR MITH SMALL SIZE DIAMONO ABRASIVES AUTHOR(SI - N.E.MILLER

$06 / 22 / 78$

$10 / 20 / 78$

$07 / 28 / 78$

$1215 / 78$

$10 / 31 / 78$

$10 / 20 / 78$

$10 / 20 / 78$

$12 / 15 / 78$ SHELF-L IFE DETERMINATION OF EPOXY.PREPREGS ANO FILM ADHESIVES

$10 / 31 / 78$ AUTHOR(S) - B.G.PARKER, C.M.SMITH

$10 / 31 / 78$ AUTHOR(SI - L.K.GILLESPIE

2112 DEBURR ING AN ANNOTATED BIBLIOCRAPHY, YOL

$11 / 15 / 78$ AUTHORISI - P.L.BLESSNER

2118 FINITE ELEMENT ANALYSIS OF LASER HELDIMG INDUCEO THERMAL SHOCK

$12 / 15 / 78$ AUTHOR(S) - K. SIDOROMICZ

2119 USE OF A DISPLACENENT MEASURING SYSTEM IFOR POSITIONING A SPHERE ON A ROTATING AXIS AND FOR MEASURING THE SPHERICAL CONTOUR

$11 / 07 / 78$ AUTHOR(S) - P.E.KLINGSPORN

MICROPRDCESSORS AS A TOOL IN DETERMININC SFERICS AND TORNADO GENESIS AUTHORISI - D.R.HITTE

$11 / 30 / 78$

2128 MICROH RESISTANCE MEASUREMENTS TO DETERMINE PLATING THICKMESS DN PHB AUTHOR (S) - H.B.ABEL

$11 / 07 / 78$

2132 ELECTROCHEMICAL DEBURRING ANOTHER SHARP SHORO IN THE BATTLE WITH BURRS AUTHOR(S) - L.K.GILLESPIE

$12 / 15 / 78$ DATA INPUT TO POP-1 1 BY MASS PARALLEL INTERFACE

$11 / 16 / 78$ 
Appendix E

AUTHOR INDEX OF REPORTS RELEASED 


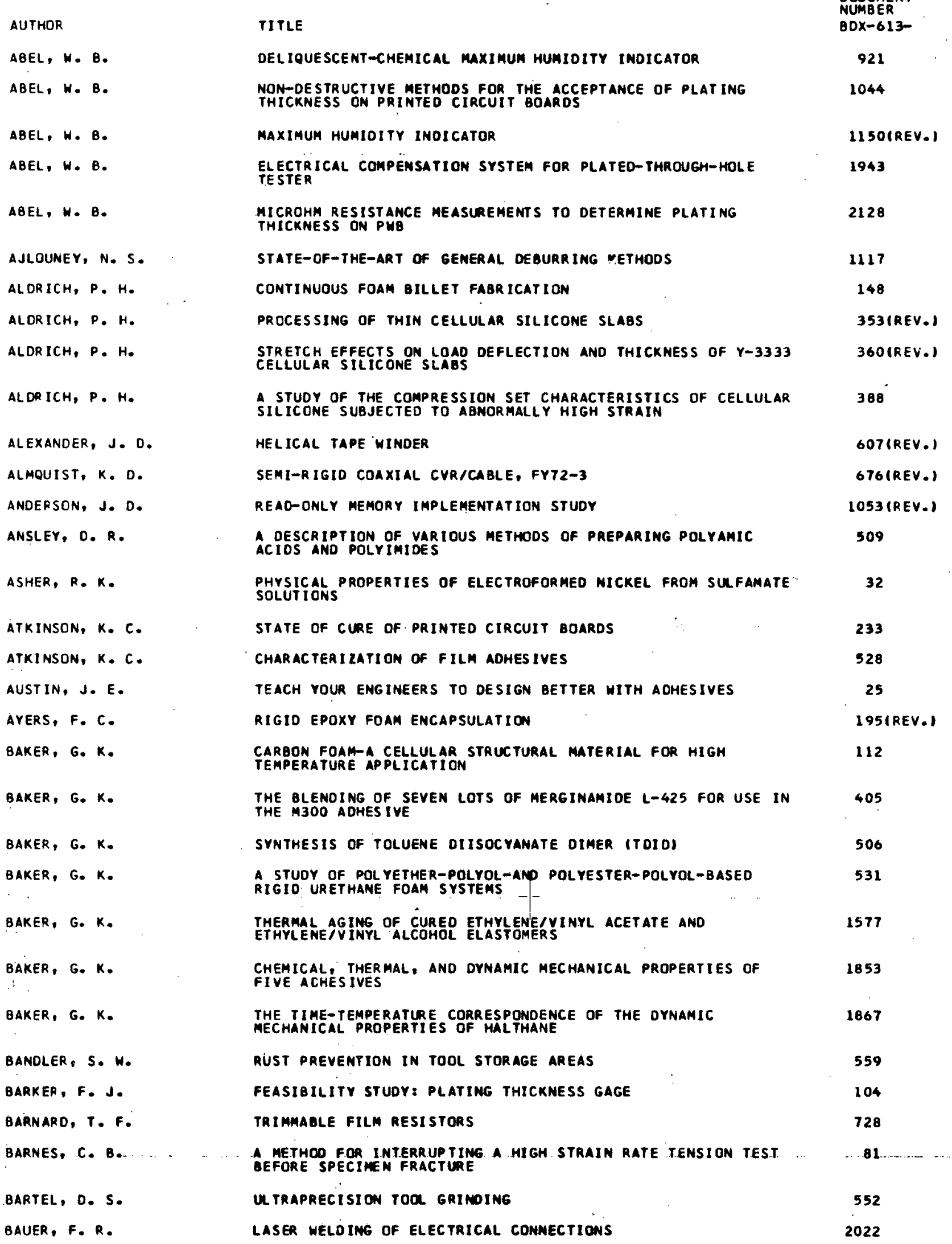


AUTHOR

BECK, W. P.

BECK, W. P.

BEGGS. R. L.

BelAROE, E.

BELARDE, E.

BELARDE, E.

BELARDE, E.

BENDURE, A. 0 .

BENEDICT, $K$. $D$.

BENEDICT, $K$. $D$.

BENEOICT, $K$. $D$.

BENGTSON, G. $W$.

BENGTSON, G. H.

BENNETT, A. G.

BENNETT, A. G.

BEOUGHER, L. C.

BERRY, JR., C. W.

BERRY, JR., C. $W_{0}$

BERRY, JR., C. H.

BERRY, JR ., C. W.

BERRY, JR., C. W.

BERRY, JR.. C. W.

GERRY, JR., C. H.

BERRY, JR., C. H.

B.ISHOP, R. D.

BLACK, P. C.

BLACK, P. C.

BLACK, P. C.

BLALEK, R. J.

BLALEK, R. J.

BLESSNER, P. L

BLESSNER, P. L.

BLESSNER, P. L.

BLESSNER, P. L.

BLESSNER, P. L.
TITLE

VISUAL AND ELECTRICAL: INSPECTION OF MINIATURE RAOARS

$B 0 x-613$

451

INTERCONMECTION TECHMOLOGY

1346

THE BEGGS MMD TEST FOR DETERMINIMG THE SHELF LIFE OF FILM ADHES IVE.

507

DEFECT DETECTION IN HIGH DENSITY BRAIDED SHIELDS

1122 (REV.)

EVALUATION OF HIGH DENSITY BRAID TERMINATION FATIGUE LIFE 1435(REV.) AND CONDIT ION

HIGH OENSITY BRAID CABLE SHIELOS

2037

RTV TOOLING EVALUATION

2038

FACE-UP BOMDING OF BEAM LEAD DEVICES

1462 (REV.)

PRODUCTION TESTING OF MAGNETIC CORE SHIFT REGISTER ELEMENTS

62

PULSE SHAPER AMO DRIVER CIRCUIT

130

DIGITIZING CONTACT BOUNCE

1756

EFFECT OF SODIUM HYOROXIDE ON ALUMINUM FOIL THICKNESS

515(REV.)

Ul trasonic melding of nYlon cell cases on a nickel caOniUm BATTERY

THE EFFECT OF RAPIOLY CHANGIHG VOLTAGES ON THICK-FILM RESISTORS

DIGITAL RLC ANALYSIS OF COU FIRESET $\times$ UNITS ICOMFERENCE PAPER I

$888($ REV.)

1085(REV.)

1945

A OIGITAL FREQUENCY MEASURING SYSTEM FOR PULSED RF DEVICES

767

INSPECTION OF FINE-PITCHED GEARS

309

INSPECTION OF FINE-PITCHED GEARS

563(REV.)

ROLAMITE SENSORS.

697(REV.)

QUALIFICATION OF MINIATURE INTERNAL THREADS USING AIR FLON

716(REV.)

VIBRATION ANALYSIS

926

WIRE WOVEN TAPE MEA SUREMENTS

1180

APPLICATION OF MOIRE TECHNIQUES TO FIXTURELESS GAgING

1213

THREAO CERTIFICATION

$1244($ REV.)

OATA INPUT TO POP-II BY MASS PARALLEL INTERFACE

2134

AN INVESTIGATION OF INTERNAL CAVITY PRESSURE FOR INJECTION-MOLDED POLYCARBONATES

PRESSURE DISTRIBUTION IN POLYCARBONATE INJECTION MOLOINGS:

AN INVESTIGATION OF INTERNAL CAVITY PRESSURE ANO THICKNESS RESPONSE FOR INJECTION MOLDED POLYCARBONATES

DEVELOPNENT OF PROCESSES FOR HYBRIO MICROCIRCUITS

CONTAINING BEAH-LEAD DEVICES

OPTIMIZATION OF LEAD FRAME BOND PARAMETERS FOR PRODUCTION OF RELIABLE THERMOCOMPRESSI ON BONDS

GOIIREV.I

951

1002

1115 (REV.)

1969

FEASIBILITY OF ELECTROPLATEO GOLD FOR HYBRIO MICROCIRCUITS 1740

HANO PUNCHING HOLES IN UMFIRED 99.5 PERCENT ALUMINA

SUBSTRATES

1461

MANUFACTUR ING PROCESSES FOR HYBRIO MICROCIRCUITS CONTAINING VIAS (CONFERENCE PAPER)

1532

HMC SPRAY, DEVELOPING, STRIPPING, ANO ETCHING

1803

ELECTROPLATEO GOLO FOR MYRIO MICROCIRCUITS

2114 


\section{AUTHOR}

BONHAM, H. B.

BONHAM, H. B.

BOSCH, A. M.

BOWDEN, H. J.

BOMERS. J. L.

BOWERS, J. L.

BOWERS, J. L.

BOWERS, J. L.

BOWLEN, D. W.

BOWLEN, D. W.

BOWLEN, D. W.

BOWLEN, D. H.

BRACKNEY, K. H.

BRADLEY, J. 0 .

BRANSTETTER, D.

BREWER, D. $H$.

BREWER, D. $H$.

BRONSON, M. J.

BROWN, E. R.

BROWN, L. W.

BRONN, L. W.

BROWN, L. W.

BRUEGGE, D. $C$.

BRUEGGE, D. C.

BRUEGGE, D. C.

BURK, P. F.

BUSHEY, C. E.

BUTLER, R. W.

CABLE, J. W.

CABLE, J. W.

CAMERON, L. S.

CAMP, C.J.

CAMP, C.J.

- CAMP, C C J. -

CAMP, C.J.

CAROER, J. H.
TITLE

BONOING DEGRADATION IN THE TANTALUM MITRIOE-CHROMIUA-GOLD METALLIZATION SYSTEM

HYERID MICROCIRCUIT INTRACONAECTION PROCESSES

1257

JOINING HIGH-STRENGTH STEELS BY OIFFUSION DONOLING

113

DENSITY MEASUREMENTS OF CURVED FOAM PARTS USING RAOIOGRAPHIC-FILN TECHNIQUE

1823

LIGHT EMITTING DIODE TESTING AND ANALYSIS

956

PHOTOMULTIPLIER TUBE TESTIN: AND ANALYSIS

1086

MULTIPURPOSE LABORATORY TEST SYSTEM APPLYIMG CAMAC STANDARDS (CONFERENCE PAPER)

1717

TELEMETRY TEST METHODS

1811

FLEXIBLE CABLE LACING

92

FLATPACK REFLOW SOLDERING

141

FLATPACK INSTALLATION BY SINGLE-PROBE REFLOM SOLDERING

326

HANO TOOL FOR INSTALL ING SOLOER FORMS OVER COMPONENT LEAOS

RESISTANCE MEASUREMENTS BY RATIO EMPLOYING VOLTAGE TO FREQUENCY CONYERTERS

DESIGN OF a MIRE TEMSIONING deVICE

FLEXIBLE CABLE LACING

SOLDERS FOR THICK GOLD PLATING

G02 (REV.)

63

844IREV.)

91

133

ANALYSIS OF THICK FILM CONDUCTOR INKS

1491

DESIGN IMPROVE MENTS FOR CVCLIC INJECTION MOLDS

1239

EFFECTS OF COATING ANO CLEANING ON CORONA AND HIGH-VOLTAGE BREAKDOWN IN ELECTRONIC ASSEMBLIES

BEAM LEAO SEMICONDUCTOR TESTING ANO FAILURE AMALTSIS

1181 (REV.)

614

975(REV.)

1275

827

FILAMENT WINDING A THIN-MALL FIBERGLASS HEMISPHERE

1239

1423

EVALUATION OF LOOP AND PARALLEL OIL CHANMEL DESIGNS

1234 (REV.)

1520

FLOW AND EXHAUST SYSTEM FOR CHEMICAL VAPOR DEPOSITION OF TUNGSTEN-RHENIUH ALLOY

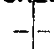

MHAT CAN THE QUALITY ENGINEER EXPECT FROM MEASURING INSTRUMENTS

57

DUAL CHANNEL MIXER

1282

PROGRAMMABLE DATA ACQUISITION SYSTEM FOR mICROMAVE CALIBRATION

1722

flexible cable lacing

91

EPON 828/MENTHANE DIAMENE CURING FACTORS

109

THE BEGGS MMD TEST FOR DETERMINING THE SHELF LIFE OF FILM AOHESIVE

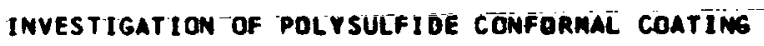

507

960(REV.)

DEPOTTING ELECTRONIC ASSEMBLIES

1530

APPLICATION OF. STATISTICAL METHODS TO MAMUFACTURING

1079 OPERATIONS 
AUTHOR

CARROLL, W. R.

CARSON, J. S.

CARSON, J. S.

CARSON, J. S.

CARTER, H.

CARTER, L. S.

CARTER, L. S.

CARTER, R. C.

CARUT MERS, D. J.

CARUTHERS, D. J.

CARUTHERS, D. J.

CARUTHERS, O. J.

CARUTHERS, D. J.

CATALANS, S.J.

CHAIT, M. J.

CHAMBER S, O. H.

CHANDLER, L. E.

CHARTOFF, R. P.

ChERAMY, E. R.

CHERAMY, E. R.

CLAGGETT, $S$. F.

ClaY, F. A.

ClaY, F. A.

ClaY, F. A.

claY, F. A.

CLAY, F. A.

CLAY, F. A.

CLAY, F. A.

CLAY, F. A.

CLAY, F. A.

CLEMENTS, D. W.

CLEMENTS, D. W.

COHAN, G. M.

COLE, B. C.

COMBS, E. E.
TITLE

COULOMETRIC OETERMINATION OF IMORGANIC MAL IDE IMPURITIES IN POLYSTYRENE AFTER DISSOLUTION IN 2-BUT ANONE

A THEORETICAL TREATMENT OF LOU VOLTAGE CONTIMUOUS SPECTRUM X-RADIATION

timesaying with a parallel amalyzing Channel attachment

an eVALUATION OF THE X-RAY fludRescence methoo of precious METAL PLATING THICKMESS MEASUREMENTS

USES OF LOW VOLTAGE $X$-RAY TUBES VITH THIM BERYLIUA WINOOWS

STATIC CHARGE AMD CURRENT LEAKAGE EFFECTS RELATING TO MANUFACTURE OF JFET ASSEMBLIES

Control of electrostatic damage to sol id state oevices

IMPROVED WORK DIRECTORS

EFFECT OF VOIDS ON THE COMPRESSIVE STRENGTH DF MOL DED RIGIO URETHANE FOAM

CHARACTER I LATION OF CONATHAME EN-4 URETHANE RESIN

COMPARISON OF ALTERMATE MATERIALS FOR MOCA CURED ADIPRENE

HYDROLYTIC STABILITY OF AOIPRENE L-100 AND ALTERMATE MATERIALS

MATER IAL AND PROCESSING CONPARISONS OF FOUR CONFORMAL COATINGS

FUNOAMENTALS OF HYDROGEN THYRATRONS

LINING OF COMPRESSION SET : TEST FIXTURES

WOVEN-MIRE TAPE VENDOR DEVELOPMENT FYT3-3

parallel gap brazing

CARBON FOAM-A CELLULAR STRUCTURAL MATERIAL FOR HIGH TEMPERATURE AP PLICATION

PERCUSSIVE ARC MELDING FOR ELECTRICM ASSEMBLIES

parallel gap brazing

LINE OF BALANCE ACCURACY

SOLID LUBRICANT APPLICATION BY SPRAY ANO TUMBLE METHODS

ELECTRODEPOSITION OF ALUMINUM

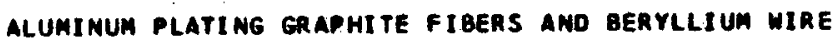

CHEMICAL YAPOR DEPOSITIOM OF MUUMIMUM

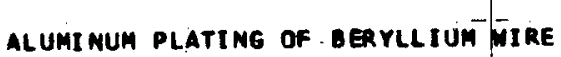

ELECTRODEPOSITION OF ALUMINUM FROM ETHER-HYDRIDE SOLUTIONS

CHARACTERI LATION OF A CHROMIUH-GOLO DEPOSITION PROCESS FOR THE PRDDUCTION OF THIN FILM HYBRIO MICROCIRCUITS

CHARACTERIZATION OF A CHOME-GOLD THIN FILM DEPOSITION PROCESS OY AUGER ANALISIS

plating hyorid micaocircuits

1284

15

994 (REV.)

1080

$964(R E V$.

532

842 (REV.)

1207

1489

1496(REV.)

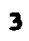

410

914(REV.)

140

112

126

140

442

108

.115

865 (REV.)

866 (REV.)

874

BTS(REV.)

1109

1173

1575

REWORK OF MULTILAYER PRINTEDHIRINGBOARO ASSEMBLIES

1315

COMPUTER AIDEO FAILGRE AMALYSIS

1369lREV.I

COMPUTERIZED QUALITY CONTROL

SYNTHESIS OF TOLUENE DIISOCYANATE DIMER (TDID)

$26^{\circ}$

506

EFFECTS OF THERMAL EXPAMSION ON EPOXY GLASS LAHIMATES 
AUTHOR

CONHAY, J. M.

CONMAY, J. M.

CONHAY, J. M.

COOPER, E. B.

COOPER, E. $B$.

COUPLAND, H. C.

COX, K. 0 .

CROCKETT, R. R.

CROCKETT, R. R.

CROMHELL, W. E.

CROMHELL, H. E.

CROMHELl, W. E.

CROMHELL, H. E.

CUMMINGS, D. A.

CUMAINGS, D. A.

DALY, J. C.

DALY, J. C.

DALY, J. C.

DALY, J. C.

DANIEL, R. A.

DANIEL, R. A.

DARBYSHIRE, H. F.

DARBYSHIRE, H. F.

DARNER, G. S.

DARNER, G. S.

DARMER, G. 5 .

DARMER, G. 5 .

DARNER, 6. 5.

Davis, 8. A.

DAVIS, B. A.

DAVIS, J. $M$.

DAYIS, JR., M. V.

DAVIS, JR., M. V.

DAVIS, JR., M. V.

DAVIS, JR., M. V.

DEGISI, S. L.
TITLE

MOISTURE EFFECTS ON THERMOSET MOLOIME COMPOUNOS

DOCUMENT

NUMBER

BDX-613-

o2L(REV.) IMPROVED DENSI TY CONTROL OF MOLOEO OESICCANTS

91O(REV.)

930 (REV.) COMPOUNOS

LINEAR TRANSDUCER TIP PRESSURE CHECKER

904

NONDE STRUCTIVE TESTING OF MELDED ELECTRICAL. CONMECTORS

CAPPING FIXTURE FOR DISCRETE SEMICONDUCTORS

1190 (REV.)

1285

NONOESTRUCTIVE TESTING OF PERCUSSIVE ARC HELOS

S6IIREV.)

BONOING STRENGTH OF EMCAPSULATING MATERIALS TO MOOKUP WIRE

135

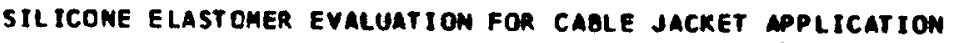

LOTIREV.) INVESTMENT CASTING TO ENHANCE PRODUCIBILITY-A CASE STUDY

291

THE CREATION OF LARGE CASTINGS OY THE INVESTMENT PROCESS

COST SAVING THROUGH APPLICATION OF THE INVESTMENT CASTING PROCESS

REDUCE THE COST OF ELECTRONIC PACKAgING hITH INVESTMENT CASTINGS ICONFERENCE PAPER)

824(REV.)

1126 (REV.)

1720

QUANTITATI VE COMPARISON OF ELECTRONIC COMPONENT-SOLDER JOINT STRESS RELIEF IN EAKAPSULATEO ASSEMSLIES

PROCESS EFFECTS ON HIEH VOLTAGE ELECTRICAL ASSEMBLIES

THERMOPLASTIC FRONTIER LAMS-GOOD ANO BAD GUYS

THIN INSULATOR SHAPES

A DESCRIPTION OF VARIOUS METHODS OF PREPARING POLYAMIC ACIOS ANO. POLYIMIDES

EVALUATION OF HIGH TEMPERATURE DIELECTRICS

STATIC COMPRESSIVE TESTING OF RIGIO POL YURETHANE FOAM

OYNAM IC RE SPONSE OF RIGIO POLUURETHANE FOAM

THIRO-OIRECTION REINFORCEMENT OF CARBONIZED COMPOSITES

SPLIT FIBERGLASS HOLOER

ULTRASONIC MELDING OF ELECTRICAL TERMINATIONS

ULTRASONICALLY MELOING ELECTRIC TERMINALS

CLOSEO CIRCUTT TELEVISION MELOTNG ALIGNMENT SYSTEM

MELOING ELECTRIC TERMIMALS ULTRASONICALLY SHIELDING EFFECTIVENESS OF SINGLE CONOUCTOR FLAT SHIELDED
CABLES

1868

1887

60

502 (REV.)

509

534(REV.)

1036 (REV.)

1583 (REV.)

144

198

IISBIREV.)

13T6(REV.)

1533

1565

1685

EFFECTS OF TEMPERATURE ON FILLEO EPOXY EMCAPSULATION MATERIALS

MONITORING SINUSOIDAL VIERATION IMVIROMMENTS MITH A TV SYSTEM

1295

THE ANALYSIS OF TIM-IRIOIUM-ITMC ALLOYS

Á METHOO FOR INTERRYPTIMG A MIGH STRAIN RATE TENSION TEST

POL MERIC CO-OEPASITION IN GOLD ELECTROPLATE

SOL OERABIL ITY PROQLENS CAUSED OY GOLO ELECTMOPLATE

324

EFFECT OF MOLECULAR STIUCTURE ON THE AGING PROPERTIES OF FLEXIBLE URETHANE FOANS 


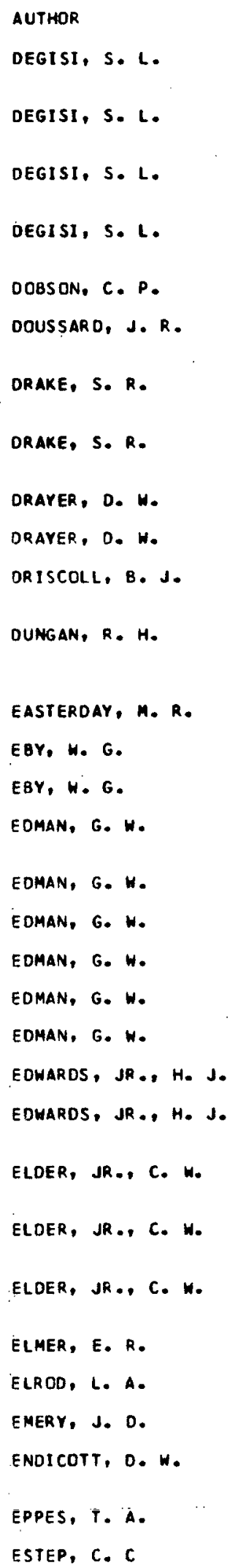

1137(REY.)

1915

724

1013

184

1373

14

88

153

45

10

BTO(REV.)

1242

374

386 (REV.)

387 (REV.)

393 (REV.)

493 (REV.)

1082 (REV.)

T76(REV.)

11921REV.I 
AUTHOR

EVANS, M. E.

EVANS, M. E.

FENOER, J. R.

FENDER, J. R.

FENOER, J. R.

FENDER, J. R.

FENDER, J.R.

FENDER, J. R.

FENOER, J. R.

FENOER, J. R.

FENDER, J.R.

FENOER, J. R.

FINUAY, 6.

FINLAY, G. $L$.

FINLAY, G. L.

F INLAY, G. L.

FINLAY, G. L.

FLOERSCH. R. H.

FLOERSCH, R. H.

FLOERSCH, R. H.

FLOERSCH, R. H.

FLOERSCH, R. H.

FLOERSCH, R. H.

FLOERSCH, R. H.

FLOERSCH, R. H.

FLOWERS, R. R.

FORMAN, G. W.

FORMAN, G. W.

FORMAN, G. W.

FORMAN, G. W.

FORMAN, G. W.

FORTE, N. V.

FOSSEY, D. J.

FOSSEY, D. J.
TITLE

SOLDER SPLATTER CAUSEO OY OUTGASSING HOLES IN PRINTEO WIRING BOARDS

EVALUATION OF ALKALINE ETCHANTS FOR PRINTEO MIRING BOARDS

1523

MEDIUM DENSITY MOLDED FOAM

209

FILLED FOAM MANUFAC TURIMG AND TESTING TECHMIQUES

252

POL YURETHAME foAM material amo PROCESS DEVELOPMENT

300

MATERIAL AND PROCESS DEVELOPMENT OF POL TURETHANE FOAH, FY71-4

485IREV.I

MIXING HEAD FOR URE THANE FOAM METERIMG NO MIXIMG MACHINES

537

MATER IAL AND PROCESS DEVELOPMENT OF POL YURETHANE FOAK,FYTZ-1

MATERIAL AND PROCESS DEVELOPMENT OF POL YURETMANE FOAM, FY72-3

MATERIAL AND PROCESS DEVELOPMENT OF POLYURETHAME FOAM,

MATERIAL EVALUATION OF POLYURETHAME FoAH, $0.05 \mathrm{G} / \mathrm{CH}$

POL YURETHANE FOAN $0.056 / C M 3$ DENSITY

553 (REV.)

718

197

1836

PROTOTYPE DIRECT MUMERICAL CONTROL SYSTEM

1964

1585

DIRECT NUMERICAL CONTROL OF MACHINE TOOLS

S4O(REV.)

EOITING CAPABILITY FOR DIRECT NUMERICAL CONTROL

1688

OVERVIEW OF DIRECT MUMERICAL CONTROL

1917

DIRECT NUMERICAL CONTROL AT BENOIX KANSAS CITY OIVISION

2018

THEORY aND OPERATION OF THE BENDIX MOLO HEATING AMO COOL ING EQUIPMENT

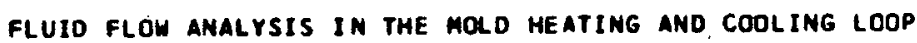

369

742

EXPER IMENTAL TESTING FOR MOLD TEMPERATURE RESPONSES

H1LIREV.)

COMPUTER SIMULATION OF THE MOLO HEATING aNo CONTROL LOOP, USING CONVENTIONAL COMTROL STRATEGIES

1764

MOLD HEATING ANO COOL ING SYSTEMS

1979

OIGITAL CONTROLLER DESIGN, AMALYSIS, AND I MPLENENTATION FOR THE MOLD HEATING AND COOLING SYSTEM

MOLD HEATING AND COOLING SYSTENS

OIGITAL CONTROLLER FOR CYCLIC TEMPERATURE CONTROL

FAST RECOVERY TIME DIOOE TESTİNG

A DISTORTION ENERGY FAILURE THEORY FOR ORTHOTROPIC MATERIALS

A STRESS ANALYSIS OF :THICK-WALL ORTHOTROPIC SPHERICAL VESSELS

OESIGN OF JOINTS FOR FILAMENT-WOUND CYLINDERS

143

DESIGN AND DEVELOPMENT OF A THREE-DIMEN
INTERLOCKED,FILAMENT- WOUND COMPOSITE

OEVELOPMENT OF A LIGHT LOAD FRICTION TESTER

1104

THIN-FILM MYBAIO MICROCIRCUIT SUBSTRATE METALLILATION

760(REV.)

VOLATILE LOSS IN POLYSTYRENE FOAM

578

869 


$$
\text { AUTHOR }
$$

FOSSEY, D. J.

FOSSEY, D. $J$.

FOSSEY, O. $J$.

FOTOPOULOS, C. U.

Fotopoulos, c. U.

FOTOPOULOS, C. U.

fotopoulos. C. U.

FOTOPOULOS, C. U.

FOULK, L. R.

FREUN, R. W.

FREUNO, R. $W$.

FREUNO, R. $H$.

FRICKE, B. 0 .

FRIEBE, E. R.

FRYE, E. R.

GALBRAITH, J. F.

GALBRAITH, J. F.

GALBRAITH, J. F.

gALLAHER, J. $B$.

GALLAHER, J. $B$.

GALLEGOS, J.

GANOUIST, H. A.

GATES, W. G.

gates, w. G.

GENSER, D. S.

GIBSON, O. L.

GIBSON, D. L.

GIBSON, D. L.

GIBSON, D. L.

GILLESPIE, L. $K$.

GILLESPIE, L. K.

GILLESPIE, L. K.

GILLESPIE, L. $K$.

GILLESPIE, L. $K$.
TITLE DESIGN MANUAL FOR POLYSTYRENE FOAM RELATING PHYSICAL.

a Nen potting mater ial-expandable polystroene bead foan

meChMNICAL PROPERTIES OF 0.2 G/CM 3 POLYSTYRENE BEAD fOAM

1647

1725

AFAP REINFORCED COMPOSITE CASE (FYTO-3)

254

HIGH TEMPERATURE THERMAL INSULATION

288 (REV.)

REINFORCED COMPOSITE CASE, FYTO-4

289 (REV.)

HIGH TEMPERATURE THERMAL INSULATION

303 (REV.)

HIGH TEMPERATURE THERMAL INSULATION

313

PRECISION FREOUENCY MULTIVIDER

882

NONMETALLIC TOOLING CAPABILITIES ANO ECONOMICS, FY72-4

738

NOMmETALLIC TOOLING CAPABILITIES and EConomics, Fy

778

NONME TALLIC TOOLING CAPABILITIES AND ECONONICS

1065 (REV.)

DESIGN MODIFICATION FOR HIGH VOLTAGE ELECTRON BEAH MELOING MACHINE

1488

INDUCTION SOLOERING EVALUATION

1453

SPIAAL WRAP: A TECHNIQUE FOR FABRICATING THICK-MALL CARBON COMPOSITES

PARALlel gap brazing

433

140

HIGH-DENSITY BRATD CABLE SHIELD, FYT2-1

543 (REV.)

HIGHDENSITY GRAIO CABLE SHIELDS, FY72-3

690

DEVELOPMENT OF AN AUTOMATEO ENCAPSULATION SYSTEM

1493

DEVELOPMENT OF AN AUTOMATEO FOAM PROCESSING SYSTEM

1691

SIMULATION MODELING OF AN AUTOMATED MATERIAL

1870

CONOUCTIVITY TESTING OR EDDY CURRENT TESTING WITH THE FM- LOOMAGNATEST CONDUCTIVITY METER

18

GLASS SEALING PROCESS FOR ELECTRICAL FEEDTHROUGHS

1424 (REV.)

CERAMIC-TO-METAL SEALING

1683 (REV.)

NONDESTRUCTIVE TESTIMG OF PERCUSSIVE ARC WELOS

107

FOCUSING PRESS URE SHDCK WAVES IN LIQUIO FOR EFFICIENT HYORDELECTRIC FORMING

THE EFFECT OF HIGHSTRAIM-RATE FORMING ON THE RECRYSTALI ZATION OF COPPER

THE PROPERTIES OF ALUMINUM AND COPPER AFTER HIGH-ENERGYTRATE FORMING

THE STRENGTHENING OF STEEL BY MARSTRAINING HITH HIGH ENERGY $\rightarrow$ RATE FORMING EQUIPMENT

ORILLING PRINTED CIRCUIT BOAROS

279

MaCHININg miniatURE SLOTS and FILLETS

576

BIOLIOGRAPHYs MACHINING hINIATURE PARTS AND MINIATURE FEATURES

585

DUBURR ING: A BIBLIOGRAPHY

593

VIBRATÖRY OEEBURRING
T35(REV.) 
AUTHOR

GILLESPIE, L. $R$.

GILLESPIE, L. K.

GILLESPIE, L. K.

GILLESPIE, L. $K$.

GILLESPIE, L. K.

GILLESPIE, L. K.

GILLESIE, L.K.

GILLESPIE, L. K.

GILLESPIE, L. $K$.

GILLESPIE, L. K.

GILLESPIE, L.K.

GILLESPIE, L. $K$.

GILLESPIE, L. $K$.

GILLESPIE, L. $K$.

GILLESPIE, L. K.

GILLESPIE, L. K.

GILLESPIE, L. $K$.

GILLESPIE, L. K.

GILLESPIE, L. K.

BILLESPIE, L.K.

GILLESPIE, L. $K$.

GILLESPIE, L. $K$.

GILLESPIE, L. $K$.

GILLESPIE. L. K.

GILLESPIE, L. $K$.

GILLESPIE, L. $K$.

GILLESPIE, L. K.

GILLESPIE. L. $K$.

GILLESP IE, L. $K$.

GILLESPIE, L. $K$.

GILLESPIE, L. K.

GILLESPIE, L. $K$.

GILLESPIE, L. $K$.

GILLESPIE, L. $K$.

GILLESPIE, L. K.

GILLESPIE, L. K.

GILLESPIE, L. $K$.

GILLESP IE, L. K.
TITLE

EXTRUDE HONE OEBURR ING

PROFILE MILLING

948

THE EFFECT DF CUTTING EDGE RAOIUS ON POISSON QURR PROPERTIES

BRUSH DEBURRING OF MIMIATURE PARTS

980IREV.)

1047

1056 (REV.)

THE EFFECTS OF REAMING. VARIABLES ON BURR PROPERTIES

1083(REV.)

PROPERTIES OF BURRS PROOUCED BY BALL BROACHING

1084(REV.)

THE BURR-MAMUFACTURING'S PERENNIAL THORN, 1112

STATE-OF-TME-ART OF GENERAL OEBURRIMG METHOOS 1117

THE MEASUREMENT OF BURMS

DEBURR ING: AN ANMOTATEO BIBLIOGRAPHY 1124

BURRS PRODUCED OY DRILLING 1248

BURRS PRODUCEO OY SIOE-MILLING CUTTERS 1303(REV.)

BURRS PROOUCED BY GRINDING. 1372

SKIVING $\quad 1429$

HAND DEBURRING OF PRECISION MINIATURE PARTS 2443

FIXTUR ING ANO HANDL ING OF MINIATURE COMPONENTS 1482

EFFECTS OF ORILLIMG VARIABLES ON BURR PROPERTIES 1502

BURRS PRODUCED BY END MILLING 1503

ECONOMIC OPTIMIZATION TECHMIQUES APPLICABLE TO PRECISION 2512 (REV.) MINIATURE MACH INING

EXTRUDE hONE OEBURR ING HITH X-BASE MEOIA 1546

DEBURRING OY CENTRIFUGAL BARREL TUMBLING 1559

MACHINABILITY AS RELATEd TO PRECISION MINIATURE PARTS 1567

DEBURRING CAPABILITIES FOR MINIATURE PRECISION PARTS 1604

$\begin{array}{ll}\text { EFFECT OF PRODUCT GEOMETRY ON BURR SILE } & 1605\end{array}$

DEBURRING: ANO ANHOTATED BIBIOGRAPHY 1616

ECONOMIC. TRADEOFFS IN DEBURRING , 1620

PRECISION OEBURRING OF MINIATURE PARTS 1697

A GUIDE TO DEBURRING, DEFLASHING AND TRIMMING EQUIPMENT 1699

ANNIHILATIING THE BURR

MACHINABILITY OF METALS AS RELATEO TO MINIATURE PRECISION 1723 COMPONENTS

PLANT-HIDE CONTROL OF DEBURRING COSTS 1738

$\begin{array}{ll}\text { BURRS PRODUCED BV TURNING. } & 1748\end{array}$

SILE EFFECTS IN CENTRIFUGAL GARREL FINISHING · 1751

EFFECTS OF DEQURRING CONTAMRANTS ON ELECTROPLATING 1797 ADHESIONS

OEBURRING CASE HISTORIES 1773

DEBURRING: AN ANNOTATEO BIBL IOGRAPHY, VOLUME IV 1777

ORILLING hINATURE HOLES PART $1 \quad \therefore \quad 1785$

DAILLING MINATURE MaLES, PART 2 
AUTHOR

GILLESPIE, L. $K$.

GILLESP IE, L. $K$.

GILLESPIE, L.K.

GILLESPIE, L. K.

GILLESPIE, L. R.

GILLESPIE, L. K.

GILLESPIE, L. $k$.

GILLESPIE, L. $K$.

GILLESP IE, L. $K$.

GILLESPIE, L.K.

GILLESPIE, L. $K$.

GILLESPIE, L. $K$.

GILLESPIE, L. K.

GILLESPIE, L. K.

GILLESPIE, L. $R$.

GILLESPIE, L. $K$.

GRACEY, J.P.

GRACEY, J. P.

GRACEY, J. P.

GRANT, S. E.

GRAY, J.L. L.

GREEN, R. M.

GR IMM, A. V.

GR INER, R. R.

GRINER, R. R.

GRINER, R. R .

GROSS, V. E.

GROTHEER, E. W.

GUzzo, v. J.

HADLEY, J.F.

HAHN, O. R.

HALE, G. J.

HALE, G... J

HALL, H. He
TITLE

DR ILLING MINIATURE MOLES; PART - 3

Box-613-

AN EXIENSION OF PROPOSED DEFIMITIONS FOR BURRS ANO

1787

RELATEDEDGE CONOITIONS

THE BURR: 1977 REPORT ON THE TECHMOLOGY OF REDUCING ITS

1791 $\cos$

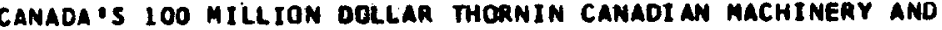
METALHORKING

MEEOED RESEARCH OM BURRS, DEBURRING, ANO EDGE FINISHING ITO BE PUBLISHEO BY SME I

HOBBING RATCHET MHEELS FOR MINATURE MECHAMISHS 1927

ELECTROCHEMICAL MACHIMING DEEP THROUGH HOLES 1948

ADVANCES IN OEBURRING 1977

YIBRATORY DEBURRIMG: AN AMLLYSIS OF COSTS IMACHINE TOOL AND 1985 BLUE BOOKI

MAND DEBLRRING: A MECESSITY THAT CAN BE IMPROVED 1986

MECHANI ZED MECHANICAL OEBURRIMG

2017

OBSERYATIONS ON IMPLEMENTING GAOUP TECHNOLOGY FOR PRECISION MINIATURE PARTS (JOURAAL ARTICLE UC-36I

2045

BARREL TUMBLING IS IT ECONOMICAL7 2067

hano deburRing a plague on the most economićal may? 2100

OEBURRING AN ANMOTATED BIBLIOGRAPHY, VOL $V \quad 2112$

ELECTROCHEMICAL DEBURRING AMOTHER SHARP SWOAD IN THE BATTLE 2132 WITH BURRS

HIGH TEMPERATURE REVERSE BIAS ANO POMER BURM-IM AT

TRANS ISTOR JUNCTION TEMPERATURES FROM ISO TO 300 DEG. C

1038

FEASIBILITY STUDY OF DIODE PRECONDITIONING

1405IREV.)

REHABILITY OF PACKAGED BEAM-LEAD TRAMSISTORS BUILT MITH DIFFERENT PRE-SEAL AS SERTIY TECMNI OUES

1952

TEN-BIt SAMPLE ANO MOLO OR PEAK hOLO CONYERTER 1066

LAPPING EQUIPMENT INSTRUAENTATION

1264

440(REV.)

.1017

LSI INTEGRATEO CIRCUIT TEST DEVELOPMENT

44

A PLANT ENGINEERING DISCIPLINE For CLEAN HORKING AREAS

54

BENEFITS ACCRUEO TO HIGHLY PREE ISE MANUFACTURE FROM CLEAM MORK PCSITIONS

APT/IGS NUMERICAL CONTRO SYSTEM 1214

BORIC ACID AMALYSIS

1601

DESIGN OF AN INSULATOR WICH MUST SURVIVE HIGH G-FORCES 213

a standardos and calibration progran in a large defense

PLAMT

COOLIMG AL UAIMUM mOLOS USING HEAT PIPES

CHEMICAL YAPOR DEPOSITION PROCESS EQUIPMENT. 269

OEPOSITION OF ALUAIMUN-COPPER ALLOY ON LANINATED POLIIMIOE 1993

SUBSTRATES FROM AN RF IROUCTANCE SOURCE

SOLOER LEVEL ING APPLIED TO MAMUFACTUAING
T6S(REY) 
AUTHOR

HANLIN, R. L. HARDING, H. B. HAROING, M. B. HAROING, W. B.

HAROING, W. B. HAROING, H. B. HAROING, W. B. HAROING, W. B. HAROING, H. B. HARDING, H. B. HAROING, W. B.
TITLE

OUANTITATIVE AUGER ELECTROM AMALYIS OF GOLO-COPPER ALLOYS THE TARNISH RESISTANCE of GOLD PLATING OVER SILVER ELECTROPLATING ON TITANIUA and TITANIUA alloys PHYSICAL PROPERTIES OF ELECTROFORMED MICKEL FROM SULFAMATE SOLUTICNS

SOLOER ING TO GOLD PLATING

WRITING METAL FINISHING PROCESS SPECIFICATIONS

GETTING MORE OUT OF PLATING THROUGH PROCESS SPECIFICATIONS SOLDERABILLITY TESTING

ELECTRODEPOSITION OF ALUMINUM

ELECTRODEPOSITING PT ANO PT-IR ALLOYS FROM MOLTEN CYANIOES: CHEMICAL PROCESS STUDIES

THE FEASIBILITY OF ELECTROPLATING PLATIMUM-IRIOIUM OUT OF FUSEO CHLORIDES

AUTOMATIC PEAK POWER MEASLREMENT OF SHDRT RF PULSES

AUTOMATIC RADAR-SENSITIVITY MEASUREMENT

ISOTROPIC ANAL YSIS OF ENRICHED BORON BY CHENICAL IONIZATION MASS SPECTROSCOPY

THERMAL SHOCK DEBURRING

A METHOD FOR INTERRUPTING A HIGH STRAIN RATE TENSION TEST BEFORE SPECIMEN FRACTURE

AN ANALOG COMPUTER for oEveloPAENT PROJECT - SCHEDUL ING

1460 (REY.) 5 24 32 40 50 61 73 115

1064lREV.)

1227

1046

1134 (REV.)

1940

642 (AEV.)

81

SOL ID STATE CONMECTOR

1479

A THEORETICAL TREATMENT OF LON VOLTAGE CONTIMUOUS SPECTRUM X-RAOIATION

AN EVALUATION OF THE X-RAY FLUORESCEACE METHOD OF PRECIOUS METAL PLATING THICKNESS MEASLREMENTS

A QUANTITATIVE STUDY DF THE SPECTRAL DISTRIBUTION OF LOW ENERGY $X-R A Y$ DEVICES

BETA BACKSCATTER MEASUREMENTS OF. ALUMINUM COATINGS ON MYLAR SUBSTRATES

SPATIAL DISTRIBUTION FROM SMALL BETA SOURCES ANO THE EFFECTS OF APERTURE SIZE

FAST NEUTRON GAUGING FEASIBILITY STUDY

DEYELOPMENT OF BETA BACKSCATTER PROBES USING MIGH-INTENSITY SOURCES (CONFERENCE PAPER)

RINC-SHAPEO BETA SOURCE IMPAOVES GAGE ACCURACY ,

STATIC ELIMINATION MORK STATIONS

PHOTOFABRICATION PROCESSES FOR PRIMTEO CIRCUITS

LIQUIO PROCESSING OF MOLOED DESICCANT

TRANSFER MOLDING OF. CLOSE-TOLERANCE PARTS.

EFFECTS OF MOISTURE AMO TRANSFER PRESSURE ON SURFACE FINISH AND SHRINKAGE OF 52-20-30 DIALLYL PHTHALATE

CONFIRMATION OF A MAJET DRILLING TECHMIQUE FOR SMALL HOLES

DESIGNING ADHESIVES AMO ADHESIVE INTERFACES FoR OPTIMUM ELECTRICAL CONDUCTIVITY 


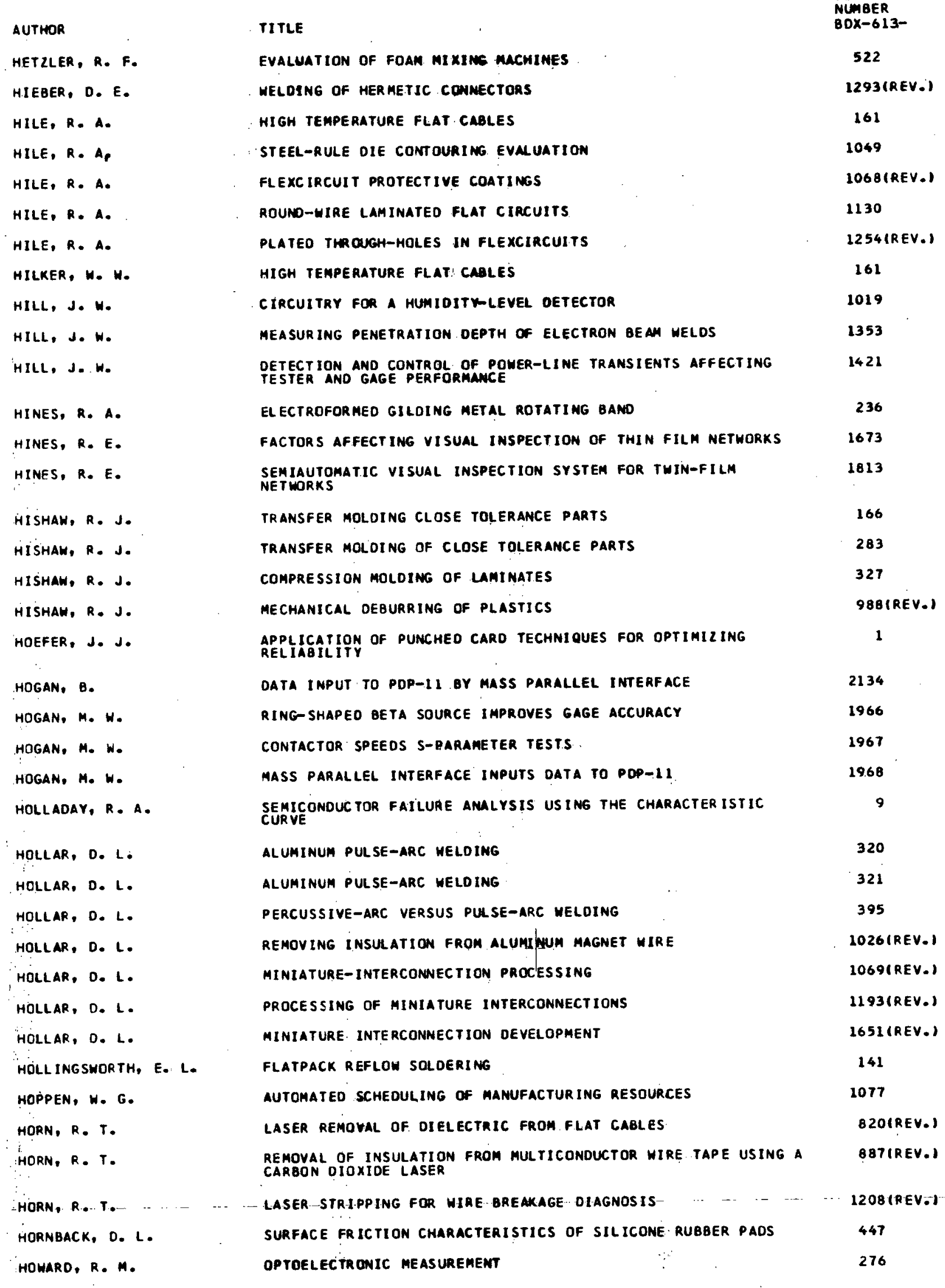




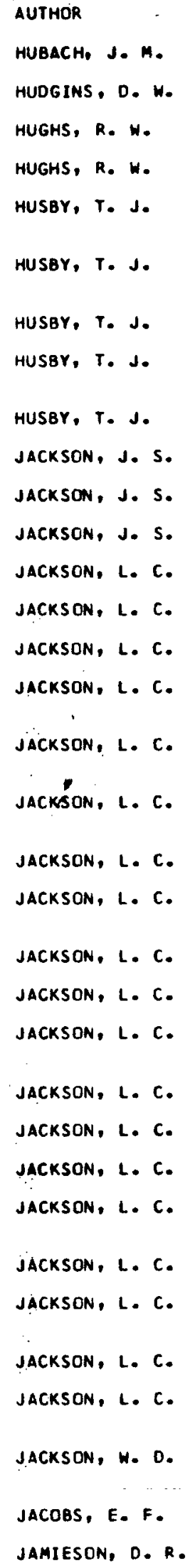

TITLE

CURE ANALYSIS OF AN AOHESIVE PRI-MER

MAINTENANCE WORK STANOAROS ANO COMPUTERILED MORK SYSTEM

IMFRARED REFLOH SOLDERING

NUMER ICALLY-CONTROLLEO ASSEMELY

CABLE FABR ICATION AND ENCAPSULATION PROCESS IMPROVERENTS, FY72-3 CABLE FABR ICATION AND ENCAPSULATION PROCESS IMPROVEMENTS,
FYT2-4

CABLE. Fabrication aNo encapsULATION, FY73-1 775

CABLE FABR ICATION ANO EMCAPSULATION PROCESS IMPROVERENTS, FY73-3

SOLOER SLEEVES

TOTAL PURCHASING OATA PROCESSING SYSTEM

WHY NOT MECHANILE MAINTENANCE

TOTAL PROCUREMENT SYSTEM PLAN

SURFACE PREPARATION OF PLASTICS FOR AOHESIVE BONDING

TEACH YOUR ENGINEERS TO OESIGN BETTER WITH AOHESIVES

general technology OF. ADHESIVES

BONOING FL UOROHALOCARBONS TO COPPER FOR ELECTRICAL APPLICATIONS

EFFECTS OF SURFACE PREPARATION ON BONO STRENGTHS OF MAGNES IUA

DESIGNING ADHESIVES AND ADHESIVE INTERFACES FOR OPTIMUM ELECTRICAL: CONDUCTIVITY

SURFACE ENERGETICS OF CONFORMAL COATINGS

SOLUBILITY PARAMETER TECHNOLOGY AND EVAPORATIVE RATE ANALYS IS USED TO EVALUATE CONTAMINANTS

MOOIFIEO PAPER HONE YCOMB AS AN ENERGY ABSORBER

COMPARISON OF METHOOS FOR CLEANING CONTAMINATEO SURFACES

SOLUBILITY PARAMETERS AMO EVAPORATIVE RATE AMALYSIS IN ORGANIC RESIDUE CHARACTERILATION

CONTAMINANT REMOVAL USING SOLUBILITY PARAMETER TECHNOLOGY

COMPATIBILITY OF CONTAMINANTS AND PLASTICS WITH SOLVENTS

REMOVAL OF SILICONE GREASE AND OLL CONTAMIMANTS IDENT IFICATION OF AN ELECTRO-NECHANICAL ASSEMBLY
CONTAMINATION PROQLEM

CONTAMINANT CLEANING FOR CRITICAL ELECTRICAL ASSEMBLY AREAS

CONTAMINANT DETECTION CHARACTERILATION, AND REMOVAL BASED ON SOLUBILITY PARAMETER PRINCIPLES

GAS PLASMA EFFECTS ON PRINTEO CIRCUIT BOARD MATERIALS

DOCUMENT

NUMBER

$B 0 X-613-$

\section{6 \\ 1235 \\ 169 \\ 804 \\ 687 \\ 740 \\ 909}

1042 (REV.)

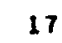

29

33

12

25

35

42

68

118

713(REY.)

B59(REV.)

$98 L$ (REV.)

1048(REV.)

$1099\left(R E V_{.}\right)$

1200

1240(REV.)

1522

1625(REV.)

1695

2028

2030

2083

CHARACTERIIZTION, AND CONTROL

A MOVING HEAD OISC OPERATING SYSTEM FOR AN 8542 A AUTOMATIC NETWORK ANALYZER (CONFERENCE PAPER)

NEM POTENTIAL IN RIGIO POTTING COMPOUNDS

1652 


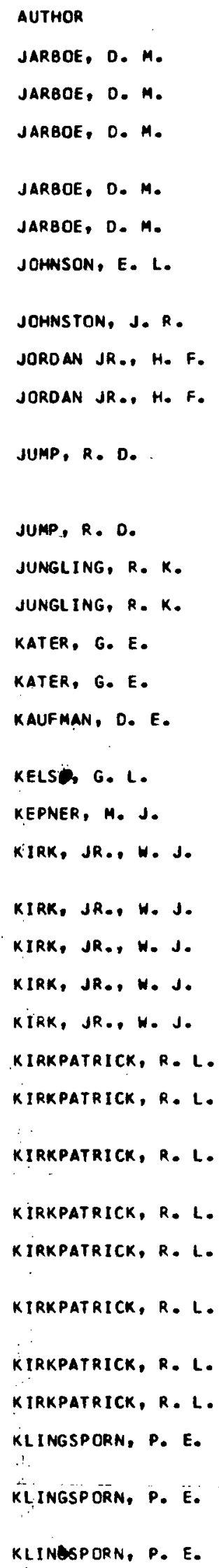

TITLE

JOINING MAGNETIC ALLOY 49FE-49CO-2Y TO 304L STAINLESS STEEL automatic lead frame plating

STRESS REL IEF TECHNIQUES FOR REDUCING THERMAL FATIGUE IN SOLDER JOINTS

SOLDERABIL ITY TEST DEVELOPMENT

$X$-RAY POLE ANALYSIS

A DESCRIPTION OF VARIOUS METHOOS OF PREPARING POLYAMIC ACIOS ANO POLYIMIDES

SYNTACTIC FOAM, FY7O-3

MINIATURE CORE ASSEMBLY FABRICATION ANO TEST PROCESSES

Fabrication AND PERformance of a laminated nonvolatile NONDES TRUCTIVE READOUT IEMORY CORE

IMPACT TESTING OF FOAMFILLEO PAPER HONEYCOMB MITH IMPACT TESTING OF FOAM-FILLED PAPER HONEYCOMB WITH REOWOOD

TECHNIQUE FOR IMPACT TESTING OF CONFINED RIGID FOAN

PHYSICAL/ChEMICAL PROPERTIES OF RIGIO LRETHAME FOAM

SYNTHESIS OF TOLUENE DIISOCYANATE OIMER (TOID)

MICROHM DECADE RESISTOR

PRECISION DC VOLTAGE REFERENCE DESIGN CONSIDERATIONS IN THE PRODUCTION OF STRIPLINE
FERRITE CIRCULATS

HIGH TEMPERATURE THERMAL. INSULATION

WORK-IN-PROCESS INVENTORY

JUNCTION.FIELO EFFECT TRANSISTOR DEGRADATION CAUSED BY ELECTROSTATIC OISCHARGE

FERRITE-CORE TRANSFORMER BONOING ANO POTTING

MAGNET-WIRE STRIPPING

CONTROL OF ELECTROSTATIC DAMAGE TO SOL 10 state deVICES

MEMORY ASSEMBLY TO HYBRIO MICROCIRCUITS

BINOMIAL SAMPLING PLANS INDEXED OY AQL AND LTPD

ONE SIOEO TOLERANCE LIMITS OF A NORMAL DISTRIBUTION BASED ON SAMPLE MEAN AND MEAN RANGE

CONFIDENCE LIMITS ON A PROPORTION DEFECTIVE CHARACTERIZED OY TWO SPECIFICATION LIMITS

CHARACTERISTICS OF SKIP LOT SAMPLING

SOLUT IONS FOR THE WAGR SEQUENTIAL T-TEST, SEQUENTIAL VARIABLES SAMPLING TO CONTROL THE PERCENT DEFECTIVE

gUANTITATI VE ADJUSTMENTS FOR PERCENT DEFECTIVES IN NON-NORMAL DISTRIBUTIONS

SAMPLE SIZE TO SET SPECIFICATION LIMITS

APPLICATION AREA fOR STATISTICAL METHOdS (CONFERENCE PAPER)

THE REFEREMCE ELECTRODE AND ITS USE IN DETERMINING INOIVIDUAL ELECTRODE POTENTIALS

REFLECTEO-LIGHT INTENSITY OISTRIBUTIONS FROM -DEFECTS-ON HIGHLY REFLECT IVE SPHERES

THE ANGULAR DISTR IBUTION OF DIFFUSE LIGHT REFLECTED FROM ISOLATED DEFECTS ON SUPERFINISHED SPHERES 


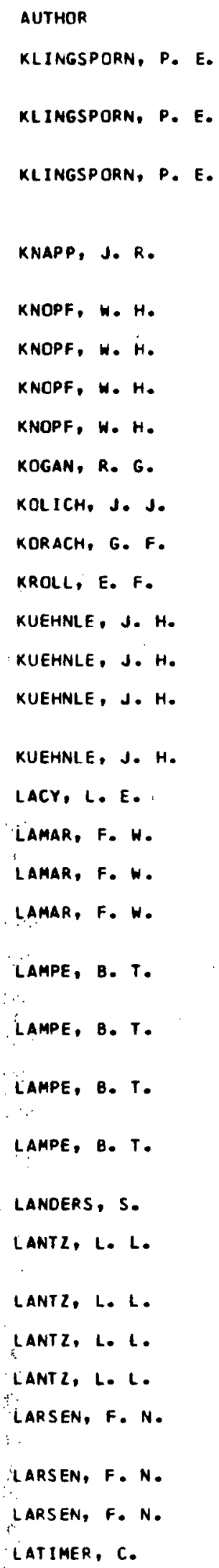

TITLE

OPTICAL SYSTEM FOR AUTOMATEO INSPECTION OF HIGHLY REFLECTIVE SPHERICAL SURFACES

FRAUNHOFER DIFFRACTION PATTEANS FROM APERTURES ILLUMINATEO WITH NONPARALLEL LIGHT

USE OF A DISPLACEMENT MEASURING SYSTEM FOR POSITIONING A SPHERE ON A ROTATING AXIS ANO FOR MEASURING THE SPHER ICAL CONTOUR

ELECTRODEPOSITION OF MICKEL-COBALT ALLOY: OPERATING PARAMETERS ANO PHYSICAL PROPERTIES OF THE DEPOSITS

IMPROVEO CURVED VACUUM BLANKETS FOR PHOTORESIST EXPOSURE

ALUAINUM ETCHANT CONTROL STUDY

ALUMINUM ETCHANT STUDIES

INPROVEO PRECISION ALUMINUM ETCHANT

EVALUATION OF CARBONYL NICKEL

RESOURCES FORECASTING SYSTEM

FILAMENT WINDING INITIAL DEVELOPMENT

A MICRODRILL LAPPING MACHINE

PROPERTIES OF PLASMA-SPRAYED ALUMINUM COATING

THERHAL SHOCK DEBLRRING

HEAT TREATMENT OF GAL-4V TITANIUM FORGINGS TO OBTAIN HIGH YIELO STRENGTH

SORTING AND HANOLING LASER TARgetS

SYNTHESIS OF TOLUENE DIISOCYANATE DIMER (TOIO)

A COEFFICIENT OF THERMAL EXPANSION CAN BE DESIGNED

TENSILE TESTING OF HIGH STRENGTH MATERIALS

STRESS-CRACKING AND FRACTURE TOUGHNESS OF POLYCARBONATE MATERIAL

REQUIREMENTS FOR REFLON SOLDERING OF INTEGRATED CIRCUIT

SOLDER SPLATTER CAUSED BY OUTGASSING HOLES IN PRINTED HIRING BOARDS

REQUIREMENTS FOR REFLOW SOLDERING OF INTEGRATEO CIRCUIT FLATPACKS

ROOM TEMPERATURE AGING PROPERTIES OF SOME SOFT SOLOER ALLOYS

SOL DER ING AUTOMATION

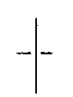

AUTOMATED ANALYSIS OF hYPOPHOSPHITE IN ELECTROLESS NICKEL SOLUTIONS

PLATING UN IFORMITY

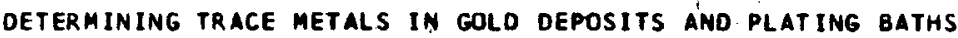

SOLUTION CONTROL FOR HYBRID MICROCIRCUIT ETCHING

MOLECULAR CHARACTERIZATION OF SILICONES BY GEL PERMEATION CHROMATOGR APHY

THERMAL. PROPERTIES OF RIGID URETHANE FOAMS

CHARACTERIZATION OF LOH DENSITY RIGIO URETHANE FOAM

APPLICATION OF STATISTICAL METHODS TO MANUFACTURING OPERATIONS
500

873

1012

121

132

1890

1497

2016

2119

66

953

955

LIOSIREV.I

1281 (REV.)

77

1078

667

102

408

642 (REV.)

872

1325

506

83

110

512

187

505

664

1599

1865

499

1079 
AUTHOR

LAUDEL, JR., A.

LAUDEL, JR., A.

LAUDEL, JR., A.

LAUDEL, JR., A.

LAZARUS, L. J.

LAZARUS, L. J.

LEE, H. H.

LEICHLITER, G. E.

LEMBKE, J. R.

LEMBKE, J.R.

LEMBKE, J. R.

LEMBKE, J. R.

LEMBKE, J. R.

LEMBKE, J. R.

LEMKE, J. R.

LENHARDT, B. W.

LENHAROT, B. W.

LEWIS, F. H.

Liou, D. H.

LIPPERT, M. C.

LOHR, R. D.

LONG, C. L.

LORMIS, F. E.

IOAMIS, F. E.

LOSURE, J. A.

losure, J. A.

LOSURE, J. A.

LUEEBERT, JR ., L. H.

LULA, J. W.

LYONS, V. A.

MAOOEN, J.P.
TITLE

Photomask measuring systems

DOCUMENT

NUMBER

BDX-613-

PLATED-THROUGH hOLES OY PLASM-ENHANCEO DEPOSITION

650

652

A RAPIO TCR TEST METHOO FOR TAMTALUH-NITRIDE RESISTIVE FILM

$1167($ REV.)

MANUFACTURING PROCESSES FOR HYBRID MICROCIRCUITS CONTAINING VIAS. (CONFERENCE PAPER)

1532

A MICROMETER SPECIMEN tRAMSLATOR FOR FlUORESCENT $x$-RAY SPECTROSCOPY

138

LOH TEMPERATURE PROPERTIES OF GENERAL ELECTRIC LEXAN BOTO-112 THIN POLYCARBOMATE FILM

A NEM TECHNIOUE FOR DETERMINING GAS OIFFUSIVITY IN HETEROGENE OUS MEOIA-PLASTIC FOAM

EFFECTS OF BDRIC ACIO ON THE CURE OF BORON-FILLED U-3602 SILICONE GUM

GRAPHIC AIO TO OESIGN OF SPLICED QUARTER-HAVE RESOMANT LINES

AN EVALUATION OF SCATTERING PARAMETER TECHNIQUES AS APPL IED TO FIELD-EFFECT TRANS ISTOR CHARACTERIZATION

AN EXPLOOING BRIDGEWIRE SIMULATOR USING THE SCEPTRE CIRCUIT ANALYSIS PROGRAM

DYNAMIC TESTING OF EXPLOOING BRIOGEWIRES

769

DIGITAL RECOROING ANO AMLYSTIS OF FAST EBW BURST WAVEFORMS

DIGITAL ANALYSIS OF EBW BURST HAVEFORMS

1381 (REV.)

PROgReSS IN AUtOMATIC IMAGE measurement

1668

1401(REV.)

CHARACTER IZATION OF THERMOCOMPRESSION BONOING PARAMETERS
FOR BEAT LEAD BONDING

1175

REMOVAL OF HYBRID MICROCIRCUIT GEAM-LEAD OEVICES FOR POSTMORTEM TESTS

1176

PRESSURE ANO TEMPERATURE GRAOIENTS IN TRANSFER MOLDING OF ELECTRONIC ASSEMBLIES

A NEW TECHNIQUE FOR OETERMINING GAS OIFFUSIVITY IN HETEROGENE OUS MEDIA--PLASTIC FOAM

ACCURACY OF WEIGHT METHOD USED TO DETERMINE ALUMINUM FOIL THICKNESS

DESIGN EVALUATION OF INSULATOR PIN

SOLDER SPLATTER CAUSED BY OUTGASSING hOLES IN PRINTED WIRING BOARDS

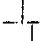

testing PRoblems of space age plastics

MECHANICAL AND PHYSICAL PROPERTIES OF ORGANIC FOAM

SHORT CIRCUIT DETECTION WITH LIQUIO CRYSTALS

OIAGNOSIS OF MALFUNCTIONS IN CONPLEX ELECTRONIC ASSEMBLIES

PRODUCTION COATING OF VIAS IN ALUMINA SUBSTRATES WITH VACUUM EVAPORATED CHROMIUM ANO GOLO ICONFERENCE PAPERI

EXTRUDE HONE DEBURR ING

TESTING THE INTERLAMIMATE ADHESION OF MULTILAYER PRINTEO WIRING BOARDS

MACHINE COOLANT SELECTION and MAINTEMANCE MaMUAL

333

229

1245 (REV.)

294

505

58

562 (REV.)

131

337 (REV.)

1535

948

2011

205(REV.)

A STUDY OF POL YETHER-POLYOL-AND POLYESTER-POLYOL-BASED

531 
AUTHOR

MARKLEY, ROE。

MARKLEY, R. E.

MARKLEY, R. E.

MARKLEY, R. E.

MARTIN, O.C.

MARTIN, R. L.

MARTINETTE, G. E.

MARTINETTE, G. E.

MARTINETTE, 6. E.

MARTINETTE, G. E.

MATZKANIN, T.

MATIKANIN, T.

mCCOY, J. C.

MCFARLAND, J. W.

MCFARLANO, J. W.

MCFARLANO, J. W.

MC ILROY, H. M.

MCILROY, H. M.

MCILROY, H. M.

MCILROY, H. M.

MC ILROY, H. M.

MCILROY. H. M.

MCILROY, H. M.

MCILROY, H. M.

MC ILROY, H. M.

MCKAY, H. P.

MCKAY, W. P.

MCKAY, W. P.

MCKAY, W. P.

MCKAY, M. P.

mCKaY, H. P.

meKaY, H. P.

MCWHIRTER, R. J.

MILLER, G.P.

MILLER, G.P.
TITLE

BeAM LEAD deVICES AND THE mIM manufactuRER

beam lead device and hyar 10 microcircuit testing

779

HANOL ING aUTOMATION AMO TEMPERATURE CONTROL ADDED TO TRANS ISTOR S- PARAMETER MEASLREMENT ICONFEREACE PAPERI

1113

1536

CONTACTOR SPEEDS S-PARAMETER TESTS

1967

HIGH FREQUENCY PARAMETER TECHNT QUES

CONDUCTIVE ADHESIVES-A SUBSTITUTE FOR SOLDER

347

7

A STUDY OF THE SHELF agING. CHARACTERISTICS OF $\mathrm{Y}-3333$ CELLULAR SILICONE

A STUDY OF THE FUNCTIONAL CAPABILITIES OF AN AIR-BEARING COMPRESSION TEST FIXTURE

A STUOY OF THE FUNCTIOMAL CAPABILITIES OF A TOTAL AREA LOADING COMPRESSION TEST FIXTURE

DEVELOPMENT OF ELASTOMER CUSHIONS

375(REV.)

390 IREV.I

391IREV.I

458 IREV.)

MEASUREMENT METHOD FOR POLYSTYRENE

355

Photogrammetric gaging

547

SPIRAL WRAP: A TECHNIQUE FOR FABRICATING THICK-WALL CARBON COMPOSITES

433

RIGID URETHANE FOAM BASED ON METHYL-ALPHA-(D)-GLUCOSIOE AND

H4OIREV.) E-CAPR OLAC TONE

CHEMICAL AMD PHYSICAL CHARACTERILATION OF A KEVLAR-FILLED EPOXY MOLDING COMPOUNO

1816

SHELF LIFE AGING OF DC-302 SILICDNE MOLDING COMPOUND

1880

CHARAC TERI ZATION OF AN EPOXY FOAM

125

FABRICATION OF POLYIMIDE SYMTACTIC FOAM PARTS

582 (REV.)

LINEAR THERMAL EXPANSION OF FILLED EPOXY RESINS

952

EVALUATION OF A HIGH TEMPERATURE SYNTACTIC FOAM

1178IREV.)

THERMALLY CONDUCTIVE SYNTACTIC FOAM

1409(REV.)

HIGH TEMPERATURE SYNTACTIC FOAM

1534

GLASS MICROBUBBLE COMPOSITE FOAM

1596

SYNTACTIC FOAM FROM SILVER COATED MICROBUBBLES

1790

EVALUATION OF CURE CYCLES FOR SYNTACTIC FOAM

1913

POL YURETHANE CABLE FABRICATION FOR SMALL QUANTITIES

HOW TO REPAIR CONNECTOR TRI-LOC̄K PINS

74

96

PREVENTING FAILURES IN SOLOERING TERMINALS

97

DESIGNING ANO MOLOING MODULE HOUSINGS FOR ELECTRONIC

122

PACKAGES

TECHNI QUES IN US ING POL YURETHANE MATER IALS F.OR ENCAP SULATION

123

exotherm location can cause problems in pott ING

124

DETERMIMING PROPER THREAD EMGAGEMENT BY MOMOCRAPH

139

CARBOM MICROSPHERE/FI BER/POLYIMIDE BINDER SÝNTACTIC

1636IREV.) COMPOSITE

AUTOMATIC MACHINE APPLICATION OF SOLIO FILM LUBRICANT

1169

IMPROVED PROCESS FOR WELDING STUDS TO RING AND STUD ASSEMBLIES 
THREE-BODY ABRASIVE WEAR WITH SHALL SILE DIAMOND ABRASIVES

FINAL REPORT ON MOTION SENSORS

EXPER IMENTAL INVESTIGATION OF A LOH YOLTAGE OPERATING MODE IN AN EPITAXIAL MESA AVALAMCHE TRANSISTOR

CMEMICAL CHARACTERI ZATION OF A POLYPHOSPHORIC ACID ETCHANT REDES IGN OF BENDIX VARIABLES DATA SYSTEM (CONFERENCE PAPER) FLATPACK SOLDERING TO SOLOER-LEVELED PRINTED MIRING BOAROS FEASIBILITY OF DRAG SOLOERING

EQUIPMENT EYALUATIONS ANO PROCESS TECHNIQUES FOR ORAG SOLDERING PRINTED WIRING ASSEMBLIES

HIGH TEMPERATURE FLAT CABLES

863

161

868

791

94

CONFORMAL-COAT ING

2078

1949

839(REY.)

IO5I (REV.)

1404(REV.)

CONFORMAL-COATING

161

257

CONFORMAL-COAT ING

265

FLOH SOLDERING AND SUPPORTING PROCESSES

286

CONFORMAL. COAT INGS

330

PROCESSING EVALUATION OF MAGNETIC PRODUCTS

341

CONFORMAL COATING FYT1-3

SLEIREV.I

SOLDER LEVEL ING OF PRINTED WIRING BOARDS

536

SOLDER LEVEL ING

443

616

SOLDER LEVELING

739

ULTRASONIC TINNING OF COMPONENT LEADS

996

STRAIN HARDENING EXPONENT FOR 17-4 PH STAIMLESS STEEL

EFFECTS OF SINTERING ATMOSPHERES ON THE ELECTRICAL AND CERAMIC PROPERTIES OF LEAD ZIRCONATE-LEAD TITANATE FERRROELECTRICS

ELECTRICAL MEASUREMENTS of PLATINg thickness

1942

INSULATION REMOVAL FROM MOVEH HIRE TAPE

1067IREV.) BORON SHOT GRADING AND INSPECTING

BORON SHOT FABRICATION

227.REV.)

EL'ECTRDCHEMICAL STUDIES OF THE GOLD-COPPER ELECTROPLATING BATH

NONLINE A COMPUTER PROGRAM FOR LEAST SOUARES ESTIMATION OF NONLINEAR PARAMETERS AND APPLICATIONS

430

813

PHYSICAL/CHEMICAL PROPERTIES OF RIGID URETHANE FOAM 106

EFFECTS OF AMINE CATALYSTS ON THE PHYSICAL PROPERTIES OF A 170 RIGID URETHANE FOAM SYSTEM

EFFECT OF AMINE CATALYST ON COMPRESSIVE PROPERTIES OF URETHAME FOAM

870

RISE-RATE TESTING OF URETHANE FOANS

941 
AUTHOR

NEET, T. E.

NEET, T. $E$.

NEWTON, K. U.

NICHOLSON, W. J.

NORWOOD, D. P.

NORWOOD, D. P.

O'DELL, G.

O DELL, G. D.

O'DELL, G. D.

OS BORNE, G. F.

OSBORNE, G. F.

oswalo, D. D.

PALMER, R. $W$.

PALMER, R. W.

PANOUS IS. N. T.

PANOUSIS. N. T.

PARKER, B. G.

PARKER, B. G.

PARKER, B. G.

PARKER, JR.. B. G.

PARKER, JR.. B. G.

PATTERSON, E. P.

PATTERSON. E. P.

pattersom, E. P.

PATTERSON, E. P.

PATTERSON, I. V.

PAULIC III, F.

PELTIER, A. J.

PETERKORT , W. G.

PETERKORT, W. G.

PETERS, J. A.

PETERSON, C. A.

PETERSON, C. A.
TITLE

PREOICTIMG COMPRESS IVE STREMGTHS IN URETHANE FOAMS AT HIGH STRAINS

EVALUATION DF SYNTACTIC POLYSULFIOE STRESS RELIEF COATINGS

PRODUCTION OF A RELAY UNDER CONTROLLEO CONDITIONS

DOCUMENT

NUMBER

$B D X-613-$

DRY MIXING OF MOLDEO DESICCANT

MANUFACTUR ING PROCESSES FOR HYBRID. MICROCIRCUITS CONTAINING VIAS (CONFERENCE PAPERI

WET FILM PHOTOLITHOGRAPHY OF HACS CONTAINING VIAS

1103 (REY.)

LOSIREY.)

2

692 (REV.)

1532

DETERMINING THE MANUFACTURABILITY OF HYBRID MICROCIRCUITS

AT MICROWA VE FREQUENCIES

A METHOD OF INSPECTING OLELECTRIC MATERIALS AT MICROMAVE FREQUENCIES

THE NECESSITY OF CHARACTERIZING HYBRIO MICROCIRCUIT ELEMENTS AT MI CROHAVE FREOUERCIES

A METHOD OF EXPOSURE CONTROL FOR ELECTRON MICROSCOPY

a SPECIMEN to FILM GaUge for the baCK REFLECTION CAMERA

A NEW DeVELOPMENT IN POL YURETHANe ELASTOMER PROCES SING WHICH REDUCES CRACX SUSCEPTIBILITY AND BUBBLE ENTRAPMENT IN-LOT VARIATIONS IN POLYSTYYRENE EXPANDABLE BEADS ANO BEAD

FILAMENT WINOING INITIAL DEVELOPMENT

1779

1582

991

1477

PROTECTIVE COATINGS FOR URANIUM-NIOBIUM ALLOYS

BONDING DEgRAOATION IN THE TANTALUM NITRIDE-CHROMIUM-GOLD METALLILATION SYSTEM

HIGH LOAD BEARING FLEXIBLE URETHANE FOAMS

1750

PREPARATION AND PROPERTIES OF BLOCKEO DIISOCYANATES

SHELF-LIFE OETERHINATION OF EPOXY PREPREGS ANO FILM ADHESIVES

PREPARATION OF a REHOYABLE POLYURETHANE ENCAPSULANT

PREPARATION OF URETHANE AOHESIVES

ELONGATION AS A QUANTITATIVE MEASUREMENT

COMPARATIVE OUCTILITY OF BRITTLE METALS BY A PRECISION ELONGATION TECHNIOUE

CASTING WARPAGE STUDY

1368

SMALL POWDER PARTS

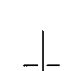

DeVELOPMENT OF INSTRUMENTATION FOR RELIABLE PERCUSSIVE arC WELDING SYSTEMS

EVALUATION OF METHODS FOR COMPUTER-AIDED DESIGN OF TEST EQUIP MENT

MASS ANODIZATION OF TANTALUH-NITRIOE RESISTORS

22

46

70

564

667

615

860

1796

2110

1406

1549(REV.)

53

92

1386(REV.)

129

349

1155IREV.I

1412(REV.)

1557

1182

AN ACTIVE LASER TRIMMER FOR PRODUCTION TRIIMMING OF FUNCTIONAL HYBRID MICROCIRCUITS

16 OFFECT OF DIISOCYANATE STR

20 
AUTHOR

PETERSON, E. E.

PETTIE, C. $B$.

PETTIE, C. B.

PIERCE, JR., R. H. $^{-}$

PIERCE, JR-, R. H.

PIERCE, JR., R. W.

PIPER, R. G.

PIPER, W. A.

PLUMB, R. S.

POPE, D. P.

POPE, D. P.

POPE, D. P.

POPE, D. P.

PQRTER, J. R.

PRESSLY, H. B.

PRIGEL, O. 0 .

QUARTERLY, B. T.

OUARTERLY, B. T.

QUARTERLY, B. T.

QUARTERLY, B. T.

QUARTERLY, B. T.

QUARTERLY, B. T.

RAHE, A. H

RAHE, A. H.

RAHE, A. H.

RATHBUN, D. A.

RATHBUN, D. A.

RAUCH, E. L.

RAUCH, E. L.

REED, D. M.

RHODES, G. H.

RICE, D. L.

RICE, D. L.

RICHARDSON, D. E. ti tLe

automatic peak poner measurement of short rf pUlses

DETERMINATION OF FOAM DENSITY

1046

180

AN EVALUATION OF THE X-RAY FLUORESCENCE METHOD OF PRECIOUS 1284 METAL PLAT ING THICKME SS MEASUREMENTS

DEPOSITION OF CHROMIUA IY RADIO FREQUENCY SPUTTERING,

651

CHARACTERI ZATION OF A CHROMIUHGOLO DEPOSITION PROCESS FOR THE PRODUCTION OF THIN FILM HYBRIO MICAOCIRCUITS

CHARACTERIZATION OF CHROMIUN-GOLD FILMS FOR HYBRIO MICROC IRCUITS

A SENSITIVE, HIGH-SPEED, OC VOLTAGE COMPARATOR

1109

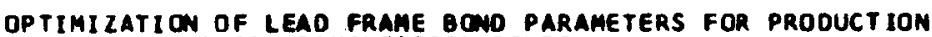
OF RELIABLE THERMOCOMPRESSION BONDS

WEAR PREVENTION IN MOLOS USED TO MOLD BORON-FILLEO

ELASTOMERS (CONFERENCE PAPER)

DEVELOPMENT OF A LIGHT LOAD FRICTION TESTER

1564 (REV.)

72

1969

1684

1104

LIGHT LOAD FRICTION COEFFICIENT AND WEAR STUDY OF THREE

1170

molding miniature plastic parts

L2121REV.1

FR ICTION ANDWEAR STUDIES FOR BONDED THIN-FILIM LUBR ICANTS

1316

INJECTION MOLOING THERMOPLASTIC RUBBER

1485(REV.)

BRAZING TITANIUM STRUCTURES

1666 (REV.)

WIGGL Y PHASE SHIFTERS ANO OIRECTIONAL COUPLERS FOR RAOIO

FREQUENCY HYBR ID MICR OCIRCUIT APPLICATIONS ICONFERENCE

1524

PAPER

MECHANICAL PRODUCTS QUARTERLY

316

ELECTRONICS ENGINEERING QUARTERLY

344

QUALITY DIVISION QUARTERLY

404

ELECTRONIC ENGINEERING QUARTERLY

431

MATER I ALS ENGINEERING QUARTERLY

460

PLASTIC PRODUCTS ENGIMEERING QUARTERLY

480

DEVELOPMENT OF A METALLOGRAPHIC METHOD FOR TUNGSTEN-RHENIUM

1744

ELECTROPOL ISHING CVD TUMGSTEN

242

ELECTROPOL ISHING OF CHEMICAL VAPOR DEPOSITED TUNGSTEN

422

ANSWERS TO LOW-COST PRODUCTION_PACKAGING

84

DESTRUCTIVE TESTING OF HYBRID MICROCIRCUITS CONTAINING

THERMOCOPRESSI ON BONDED DEVICES AND LEAD-INDIUH SOLDERED CAPAC ITORS

2081

DETERMINATION OF SEMICONDUCTOR JUNCTION PROF ILES BY ANGLE LAPPING OF SEMICONDUC TOR WAFERS

604 IREV.)

SURFACE EFFECTS ON SEMICONDUCTORS

1468

A TRANSISTORILED HIGH INPUT IMPEDAMCE AMPLIFIER

13

51

THE DETERMINAT
STANDARD CELL

HOT GAS MOLOING OF POLYSTYRENE FOAM

535

HIGH-DENSITY POLYSTYREME BEAD PREPARATION

L199IREV.I

PULSE SHAPER AND DRIVER CIRCUIT

130 
AUTHOR

RICHARDSON, W. E.

RICHAROSON, W. E.

RICHARDSON, H. E.

RICHAROSON, W. E.

RINKER, C. D.

RINKER, $C$. D.

ROBB, J. M.

ROBB, J. M.

RDBB, J. M.

ROBBINS, G. V.

ROBBINS, G. V.

ROBINSON, L. A.

ROBINSON, R. D.

RODE, J. J.

RODE, J.J.

ROEBUCK, C. E.

POLFE, E.J.

ROME, C. P.

RUSSELL, R. J.

RUSSELL, R, J.

RUSSELL; R. J.

RUSSELL, R. J.

RUSSELL, R . J.

RUSSELL, R. J.

RUSSELL, R, J.

RUSSELL, T. J.

RUSSELL, T. J.

RUSSELL, T. J.

RUSSELL, T.J.

RUSSELL, T. J.

RUTHERF ORD, W.

RUTHERFORD, W. C.

RUTHERFORD, W. C.

SADLER, R. L.

SALARY, J.

SALARY, J.
TI TLE

THE SYMTHESIS OF A LOW-DENSITY AIGID EPOXY FOAN

SYNTHESIS OF TOLUENE DIISOCYANATE OIMER (TDID)

407

LOW DENSITY FOAMS FOR VACUUM INSULATION

506

938

PREPARATION OF A CTBN-MODIFIED EPOXY RESIN

1121IREV.I

ECONONIC IMPROVEMENT OF INVENTOAY SAMPLING

885

CONTINUOUS STRATIFIED SAMPLING OF IN-PROCESS INVENTORY

1032

AFT SUPPORT MACHINED FROM TITANIUM

525

OPTIMIZING MINIATURE CYLIMORICAL PIECEPART MACHINING

CAPABILITIES

PROCESS ACCEPTANCE AMD ADJUSTMENT TECHNIQUES FOR SHISS AUTOMATIC SCREW MACHINE PARTS

708IREV.)

1354(REV.)

RADIUS GENERATION IN VIBRATORY OEBURRING 85

RADIUS GENERATION IN VIBRATING FINISHING

CARRIER SYSTEM FOR TESTING ANO CONDITIONING OF BEAM LEAD 21708 DEVICES

ELECTROFORMED NICKEL MOLD INSERTS

454

DEgRAdATION OF gLASS VACUUM TUBES AS a RESULT OF HELIUA PERMEATION

PERMEATION OF HELIUN INTO VACUUM TUBES

30

VOLATILE LOSS IN POLYSTYRENE FOAM

578

DEVELOPMENT OF A COMPARATIVE ANALYZER FOR 2060 MI CROPROCESSOR

GROUP TECHNOLOGY

1478

SMALL ANGLE GENERATOR

111

DIfFERENTIAL PRESSURE MERCURY MAMOMETER

878

MULTILAYEREO PLATING THICKNESS MEASUREMENT

1632 IREV.)

DEVELOPMENT OF PLATING THICKMESS STANDARDS FOR THE PHYSICAL VAPOR DEPOSITION OF ALUMI NUM ON KAPTON

DEVElopment DF PLATING ThICKMESS STANDARos

1634

1709

MULTILAYERED PLATING THICKNESS

1795

PLATING THICKNESS STAMOARDS

1805

A VARIABLE PHASE, CONSTANT VSMR, ANTENNA LOAD

the impeoance of a coIl placé- ON a conducting plane 27

RAOAR ANTENNA TEST LOOAD 28

DESIGN OF A VARIABLe Phase, CONSTANT YSMr, micromave lOAD 79

A TECHNIQUE FOR MINIMIZING SLOTTED LINE MEASUREMENT ERROR 86

DETERMINING THE MANUFACTURAB ILITY OF HYBRIO MICROC IRCUITS 1582 AT MICROHAVE FREQUE WCIES

335

712

fabRICATION DF FAILSAFE PRESSURE VESSELS

508

AN EVALUATION OF COMMERCIAL POLYARYL POLYISOCYANATES IN A BENDIX RANSAS CITY FOAM SYSTEM

1110 
AUTHOR

SALARY, J.

SCHANT Z, L. E.

SCHANT Z, L. E.

SCHANTZ, L. E.

SCHANT Z, L. E.

SCHANT Z, L. E.

SCHANTZ, L. E.

SCHANT Z, L. E.

SCHANTZ, L. E.

SCHANTZ, L. E.

SCHILLING, 0.6 .

SCHLESS ELMAN, L.A.

SCHMIOT, A. F.

SCHMIDT, R, E.

SCHMIDT, R. W.

SCHOEPPNER, M. P.

SCHOEPPNER, M. P.

SCHOEPPNER, M. P.

SCHUSTER, V.E.

SEESE, H. J.

SETTER, D. L.

SETTER, O. L.

SETTER, R. J.

SHEELEY, J. 0 .

SHEELEY, J. D.

SHEELEY, d. D.

SHEELEY, J. D.

SHEELEY, J. D.

SHEETS, L.

SHEETS, L. R.

SHEPHERD, O. E.

SMERMAN, D. M.

SIDOROWICZ, $K$.

SIDOROWICZ, $K$.

SIEGGEN, E. M.
TITLE

FLEXIBLE POL YURETHAME FOAM

PHOTORESIST CHARACTERIZATION METHOD FOR HYBRIO MICROCIRCUITS

HYBRIO MICROCIRCUIT, PHOTOLITHOGRAPHIC PROCESS

MASK GENERATION

1345(REV.)

653

678

959(REV.)

AUTOMATIC PHOTOMASK ALIGNMENT ANO EXPOSURE ON 3.75 BY 4.50 INCH SUBSTRATES

HOI (REV.)

SPRAY PHOTORES IST DEVELOPMENT DN MULTIPLE HYBRIO MICROCIRCUIT SUBSTRATES

1148

INFRARED BAKING OF POSITIVE PHOTORESIST FOR HYBRID MICROCIRCUIT FABRICATION

PHOTORESIST APPLICATION BY RQLER COATING

1246

MULTIPLE HYBRIO MICROCIRCUIT PROCESSING

1288

1383

AUTOMATIC LABORATORY DATA ACQUISITION SYSTEM

1566

SCS CASE

463

AUTOMATIC MEASURING OF TEMPERATURE COEFFICIENTS

36

67

a single pulse peak reading voltmeter

1235(REV.)

USE OF ENT TO ENCAPSULATE AMALYZER ASSEMBLIES

1144 (REV.)

DETECTION AND SUPPRESSION OF TRANSISTOR OSCILLATION DURING POWER-BURN-IN

INTERFEROMETER PRISM: PDR 187

127

TESTING FOR MATERIAL FLAWS ANO THERMAL DESIGN DEFECTS USING HOLOGRAPHIC INTERFEROMETRY

HOLOGRAPHIIC INTERFEROMETRY

the impedance of a coll placed on a conducting plane

FINE-EDGE BLANKING AND PIERCING

612

ONC COMMITTEE FINAL REPORT

MOORE NUMBER 5 dIAMOND TURNING MACHINE DESIGN AND DESCR IPTION

ELECTRICAL CONDUCTIVITY STUDY OF ETCHED-OUT PLATED-THROUGH HOLES IN DOUBLE-SIDED PRINTED WIRING BOARDS

FLUIDIZED BED COATING

autoclave fabrication teChnIQUES for flat Cables

OEVELOPMENT OF A SELF-CONTAINEL LAMINATING FIXTURE

OEVELOPMENT OF A FORMING STATION

DEVELOPMENT OF AN IMPROVED BONDING FIXTURE

COMPUTER APPLICATIONS ANO HYBRID MICROCIRCUITS

967(REV.)

27

366

376

2051

1434

100

579(REV.)

927 (REV.)

1123 (REV.)

1252 (REV.)

2020

INPUT ANO OUTPUT DEVICES FOR A COMPUTERILEO MASK-MAKING SYSTEM

654

1988

RESIN PROCESS CONTROLS

1873

OEPOSITION AND CHARACTERIZATION OF
ALUMINUM. FILM ON KAPTON LAMINATES

FINITE ELEMENT ANALYSIS OF LASER INDUCEOTHERMAL SHOCK

2044

FINITE ELENENT ANALYSIS OF LASER WELOING INDUCED THERMAL
SHOCK

2118

AUTOMATIC MEASURING OF TEMPERATURE COEFFICIENTS

36 
AUTHOR

SIEVER, L. W.

SIM, J. R.

SIMPSON, H. E.

SMITH, C. H.

SMITH, C. H.

SMITH, C. H.

SMITH, C. $H_{0}$

SMITH, C. H.

SMITH, C. H.

SMITH, C. H.

SMITH, C. H.

SMITH, C. H.

SMITH, C. H.

SMITH, C. H.

SMITH, C. H.

SMITH, C. H.

SMITH, C. H.

SMITH, C. H.

SMITH, C. H.

SMITH, C. H.

SMITH, C. H.

SMITH, C. H.

SMITH, C. H.

SMITH, D. D.

SMITH, O. D.

SMITH, F. M.

SMITH, F. R.

SMITH, J. C.

SMITH. J. C.

SMITH, L. H.

SMITH, L. W.

SMITH, L. W.

SMITH, R. M.
TITLE

STRENGTH MEASUREMENTS ON TRANSISTOR HEADER LEAD MELOS 871

INTERCONNECTION TECHNOLOGY 1346

REPORT ON DEVELOPMENT OF INDUCTANCE STANDARDS 98

EFFECT OF DIISOCYANATE STRUCTURE ON LOAO-BEARING PROPERTIES 16 OF FLEXIBLE URETHANE FOAHS

CAST FLEXIBLE URETHANE POLYMERS 20

RIGIO URETHANE FOAMS FROM E-CAPROLACTONE POLYESTERS 38

AN EPOXY RESIN WITH IMPROVED ELECTRICAL PROPERTIES 41

A CAST FLEXIBLE ESTER-URETHANE POLYMER 65

THERMAL CHARACTERISTICS OF SOME RIGID URETHANE FOAMS 69

EFFECT OF MOLECULAR STRUCTURE ON THE AGING PROPERTIES OF. 75 FLEXIBLE URETHANE FOAMS

STRUCTURAL APPLICATION CHARACTERISTICS OF A RIGID URETHANE FOAM SYSTEM

BORON TRIFLUORIDE-AHINE ADDUCTS AS HARDENERS FOR LIQUIO EPOXY RESINS

PHYSICAL/ChEMICAL PROPERTIES OF RIGIO URETHANE FOAM 106

CHARACTERIZATION OF AM EPOXY FOAM 125

THERMAL PROPERTIES OF RIGID URETHANE FOAMS 132

THERMAL PROPERTIES OF LACTONE ESTER-BASED RIGIO URETHANE 406 FOAMS

A STUDY OF POL YETHER-POLYOL-AND POLYESTER-POLYOL-BASED 531

RIGID URET KANE FOAM S YSTEMS

532

EFFECT OF VOIDS ON THE COMPRESSIVE STRENGTH OF MOLDED RIGIO

532

PREPARATION ANO PROPERITES OF DEUTERATED POL YETHYLENE

1570

THERMAL AGING OF CURED ETHYLENE/VINYL ACETATE AND 1577

ETHYLENEIVINYL ALCOHOL ELASTOMERS

A NEW POTTING MATERIAL-EXPANDABLE POLYSTYRENE BEAD FOAM 1647

IMPROVED BONDABILITY OF MOLDED RIGIO URETHANE FOAM BY 1915

PLASMA TREATMENT

SHELF-LIFE DETERMINATION OF EPOXY PREPREGS AND FILM ADHESIVES

COMPUTERILED EXOTHERM CALCULATIONS FOR LARGE EPOXY CASTINGS

SYNTACTIC. FOAM EVALUATION

SURFACE TEXTURE-MEASUREMENT OR CONFUSION

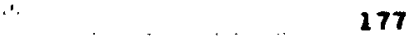

CHARACTERI ZATION OF THIN FILM MICROSTRIP ATTENUATORS AT

MICROWAVE FREOUENCIES

THE DETERMINATION of aVERAge CyCle time for an automateo WAREHOUSE

COMPUTER SIMULATION MODELING OF AN AUTOMATEO WAREHOUSE SYSTEM

BEAM LEAD SEMICONOUCTIOR TESTING ANO HANOLING

2110

114

177

89

1857

274

456

194

BEAM LEAD SEMICONOUCTOR TESTING ANO FAILURE ANALYSIS

AUTOMATIC TESTING AND HANDLING OF BEAM LEAD TRANSISTORS AND 1081 OIODES

THERMAL AGING OF CURED ETHYLENE/VINYL ACETATE AND

ETHYENETVINYL ALCOHOL ELASTOMERS

1577 
AUTHOR

SMITH, R. M.

SMITH, R. S.

SMITH. R. 5 .

SMITH, V. V.

SNELT, R. B.

SPIES, F.A.

STABLEIN, JR., P. F.

STABLEIN, JR ., P. F.

STAGNER, R. $T$.

STARKEY, J. P.

STARKEY, J. P.

STARKS, O. M.

STEELE, J. W.

STEELE, J. W.

STEELE, J. ...

STEELE, J. W.

STEGMAIER, T. F.

STEIN, J. D.

STEVENS, C. A.

STEVENS, C. A.

STEVENS, C. A.

STEVENS, C. A.

STiLES, R. W.

STILES, R. W.

STILES, R. W.

STILES, R. $H$.

STILES, R. W.

STIMETL, C. J.

STIMETZ, C.J.

STIMETL, C. J.

STITTSHORTH. D. E.

STITTSWORTH, D. E.

STITTSWORTH, D. E.

STITTSWORTH, D. E.

STITTSHORTH, D. E.
TITLE

THERMAL AGING OF CURED ETHYLENE/VIMYL... ELASTOMERS (EXPANDED VERSION OF BOX 613-1577)

COMPUTER AIDED FAILURE AMALYSIS

COMPUTER AIDED FAILURE ANALYSIS

FINAL REPORT ON MOTION SENSORS

SOLDER SPLATTER CAUSEO BY OUTGASSING HOLES IN PRINTED WIRING BOARDS

COMPOSITE CHARACTER ISTIC OF TMO TUNAEL DIODES IN SERIES

CVO SCALING STUDY

ELECTROPOL ISHING OF CHEMICAL VAPOR DEPOSITEO TUNGSTEN

EQUIPMENT FOR a LASER SURFACE MEASURING SYSTEM

a micrometer SPECIMEN TRANSLATOR fOR fLUORESCENT X-RAY SPECTRCSCOPY

IMPROVED OXYGEN ANALYSIS USING A THALLIUM ACID PHTHALATE (TAP) CRYSTAL (CONFERENCE PAPER)

DETERMINATION OF GOLD-COPPER ALLOY COMPOSITION BY X-RAY FL UORE SCENCE ANALYSIS

STATE OF CURE OF PRINTED CIRCUIT BOAROS

1626

1552 (REV.)

2006

791

505

31

414

422

1848

138

1622

1716

233

SPIRAL WRAP: A TECHNIQUE FOR FABRICATING THICK-WALL CARBON COMPOSITES

433

PROTECTION OF GLASS TUBES IN EPOXY ENCAPSULATION

931 IREV.I

N-METHYL-2-PYRROLIDONE AS A STRIPPER FOR PLASTIC POTTING COMPOUNDS

COMPRESSION MOLOING DF LAMIMATES

1955

327

HIGH TEMPERATURE NOMPLAMAR LAMIMATES

1783

FOCUSING PRESSURE SHOCK MAVES IN LIQUID FOR EFFICIENT HYOROELECTRIC FORNING

THE EFFECT OF HIGH-STRAIN-RATE FORMING DN THE

RECRYSTALI ZATION OF COPPER

THE PROPERTIES OF ALUMIMUM AND COPPER AFTER

HIGHENERGYTRATE FORMING

THE STRENGTHENING OF STEEL BY MARSTRAINING WITH HIGHENERGYTRATE FORMING EQUIPMENT

SHAPING RATCHET TEETH

GRINDING MINIATURE PINION GEARS 1007

ELECTRCCHEMICAL GRINOING

GEAR CUTTING 1392

SLOTTING BY ELECTROCHEMICAL GRIMOING 1908

ELECTRDDEPOSITION OF ALUMI MUM

GOLD PLATING 805

PLATING ON POLYIMIOE

1177 (REV.)

ELECTRICAL TERMINATIONS: WELOED, BRAZEO AND BONDEO 427

ELECTRICAL TERMINATIONS: WELDED, BRALED, AND BONDED, FYTI-4 4.72

ELECTRICAL: TERMINATIONS WELDEO, BRAZEO AND BCNOED, FY72 1

ELECTRICAL: TERMINATIONS: MELDED, ERAZED AND BONDEO, FV72-2.

613IREV.I

BENDIX KANSAS CITY ELECTRICAL TERMIMATION WELOING HANOBOOK 
AUTHOR

STITTSMORTH, D. E.

STITTSHORTH, D. E.

STITTSHORTH, D. E.

STOLTL, D. L.

STONE, D. W.

STONE, D. $W$.

STONE, W. J.

SWAFFORD, J. H.

SWAFFORO, J. H.

SHANSON, G. 0 .

SWANSON, G. D.

SWARTL, G. A.

SWARTZ, G. A.

SHART Z, G. A.

SHISHER, P. L.

SWOBOOA, R. C.

SWOBODA, R. C.

TAYLOR, J. L.

TAYLOR, J. L.

TAYLOR, J. L.

TAYLOR, J. L.

TAYLOR, R. D.

TAYLOR, R. $D$.

TAYLOR, R. D.

TAYLOR, R. 0 .

TAYLOR, R. $D$.

TAYLOR, R. D.

TAYLOR, R. D.

TAYLOR, R. D.

TAYLOR, R. F.

TAYLOR, R. F.

TAYLOR, R. F.

TELLEZ, R. M.

THOENI, A. K.
TITLE

ELECTRICAL TERMIMATIONS: MELOED, BRAZED, AND BONDED, FYT2-3

ELECTRICAL TERMINATIONS: MELOEO, BRAZEO, AND BONDED, FY72-4 ULTRASONIC WELDING OF ELECTRICAL TERHIMATIONS

QUANTITATIVE AUGER ELECTRON ANALYSIS OF GOLO-COPPER ALLOYS

IMPROVEO RAOLO-FREQUENCY POMER STANDARDS

LOW FREQUENCY SHIELOING OF AN EXPERIMENTAL MULTIWIRE CABLE

NONDESTRUCTIVE TESTING OF PERCUSSIVE ARC WELDS

APPLICATION OF PUNCHEO CARD TECHNIQUES FOR OPTIMIZING RELIABILITY

FAILURE MOOES OF BEAM LEAD SEMICONOUCTORS IN THIN FILM HYBRID MICROCIRCUITS ICONFEREMCE PAPERI

DESIGN MANUAL FOR POTTING ELECTRONIC ASSEMBLIES

MEASURING STRESS IN LEAD WIRES

THICK-FILM NETHORKS

A SUSPENDED METAL. MASK FOR THICK-FILM PRINTIMG

EFFECTS OF GEOMETRY ON THICK-FILM RESISTORS

PREVENTION OF TIN-LEAD OVERMAND IN PLATEO COPPER-CLAD

PRINTEO WIRING BOARDS

FEASIBILITY STUDY OF PRODUCTION OF 0.24 G/CM3-DENSITY POLYSTYRENE BEAD FOAK

FEASIBILITY STUDY FOR PROOUCING HIGH-OENSITY POLYSTYRENE BEAD FOAM

THE aUtOMATIC bIAS ANo flUX-RESET OF A MAGNETIC MOdULATOR

DESIGN CONSIDERATIONS IN THE PRODUCTION OF STRIPLINE FERRITE CIRCULATORS

AN AUTOMATIC RF-NETWORK ANALYZER AS A SUPPORT TOOL FOR ENGINEER ING AND MANUF ACTURING

WIGGL Y PHASE SHIFTERS AND DIRECTIONAL COUPLERS FOR RAOIO FREQUENCY HYBR IO MICRDC IRCUIT APPLICAT IONS (CONFERENCE PAPERI

RAPID VISUAL SCANNING SYSTEMS FOR PCB'S, FYTI-2

RAPID VISUAL SCANNING SYSTEMS FOR PCB'S, FYT2-1

DETERMINATION OF HOLE LOCATION ON PRINTED CIRCUIT GOAROS

PANTOGRAPH POSITIONING SYSTEM FOR INSPECTING MICROCIRCUITS

OMNI-OIRECTIONAL ILLUMINATION FOR PRINTED CIRCUIT BOARO INSPECTION

AUTOMATEO VISUAL INSPECTION SYSTEM FOR PRINTEO WIRING ASSEMBLIES

AUTOMATED TESTING IN A HIGH MOISE ENYIRONMENT

RAPID VISUAL SCAMNING

FILLED PAPER HONEYCOMB

INVESTIGATION OF KNITTED FABRICS EFFECTS OF VARYING FORMULATIONS OF THREE TOI POLYURETHANE
FOAMS

A MOVING HEAD DISC OPERATING SYSTEM FOR AN. 8542A AUTOMATIC NETHORK ANALYZER (CONFERENCE PAPER)

CARGON FOAM-A CELLULAR STRUCTURAL MATERIAL FOR HIGH TEMPERATURE APPLICATION
527

538

814

932

DOCUMENT NUMBER

BOX-613-

662

773

1016

1466(REV.)

971

126TIREV.I

107

1

1456

1216(REV.)

1775

363 (REV.)

648

649

670

1298 (REV.)

1422(REV.)

59

146

1297(REV.)

1524

942

1076(REV.)

1138

1187

190

783

1137 (REV.)

1652

112 
AUTHOR

THOENI, A. K.

THOMSON, C. E.

THORNE, L. F.

THORNE, L. F.

THORNE, L. F.

THORNE, L. F.

THORNE, L. F.

THORPE, H. G.

TRENT, M. A.

TSCHECHTELIN, L. F.

TURNER, W. H.

ULITCHNY, M. G.

ULITCHNY, M. G.

ULITCHNY, M. G.

URIBE, F.

USDOE,

VAJDIC, J. C.

VANCLEVE, R. A.

WADOELL, M. L.

WACDELL, M. L.

WAGNER, R. $D$.

WAGNER, R. D.

WALKER, F. A.

HALKER, F.A.

HALKER, F. A.

- HALKER, F. A.

HALKER, J. M.

HALKER, J. M.

WALKER, J. M.

HALKER, J. M.

WALTER, C. L.

WALTER, C. L.

WALTER, C. L.

WALTER, C. L.

WALTER, C. L.
TI TLE

BENDIX KANSAS CITY ELECTRICAL TERMIMATION MELDING HANDBOOK

A SLOW TIME GASE READOUT SYSTEM FOR MON-REPETITIVE PULSE ANALYSIS

THE DESIGN AND aDVANTAgES OF AN AIR-ACCELERATED IMPACT MECHANICAL SHOCK MACHINE

DESIGN OF AN INSULATOR WHICH MUST SURYIVE HIGH G-FORCES

IMPACT TESTING OF MATERIALS USING AN EIGHT-INCH AIR GUN AND COMPUTER REDUCTION OF DATA

IMPACT TESTING DF FOAH-FILLED PAPER HONEYCOMB HITH ENERGY-ABSORPTION CHARACTERISTICS COMPARED TO BALSA AND REDWOOD

TECHNIQUE FOR IMPACT TESTING OF CONFINED RIGIO FOAM

THE ANALYSIS OF AN INPUT BUFFER FOR REMOTE CONTROL OF LOGIC CIRCUITS

TEMPERATURE VERSUS TIME CURVES fOR MANUAL AND AUTOMATEO SOLOER ING PROCESSES

637

71

52

213

944 (REV.)

946 IREV.)

1059(REV.)

64

1920

MODERN GAGE CONTROL

39

128

LEAO WIRE SOLDERABILITY TEST

1454(REV.)

1531(REV.)

FRACTURE MECHANICS TESTING CAPABILITY

1812

MECHANICAL AND MICROSTRUCTURAL PROPERTIES CHARACTERIZATION OF HEART-TREATED BETA EXTRUDED TI-6AL-6V-2SN

LASER CONTOLRED AMD DRILLED SUBSTRATES FOR THICK FILM APPLICATIONS

1563 (REV.)

ENVIRONMENTAL SUMMARY FOR CALENDAR YEAR 1977

1987

CONTROL SYSTEM MODELING OF A CENTR IFUGE (CONFERENCE PAPER)

1950

LASER CUTTING OF KEVLAR LANINATES

1877

CLEANING AND ASSEMBLY TECHNIQUES, FY73-1

793 (REV.)

CONTROL OF ELECTROSTATIC DAMAGE TO SOLID STATE DEVICES

ELECTRICAL CONNECTION STUDY

1080

204

ALUMINA LAPPING abRaSive STUdY

1692

HRITERS GUIDE TO THE PREPARATION OF ARTHORK FOR TECHNICAL REPORTS

THE EDITOR IS NOTEBOOK

234

259

TYPIST'S GUIDE TO BENOIX, KANSAS CITY TECHNICAL. REPORTS

THE INCOMPLEAT STYLIST

261

317

TEMPERATURE DEPENOENT PROPERTIES OF POTTING MATERIALS

1133 (REV.)

DIELECTRIC ANALYSIS OF AN EPOXY PREIMPREGNATED MATERIAL

1396

LOW DENSITY EPOXY ENCAPSULANTS

1411(REV.)

TEMPERATURE DEPENDENT PROPERTIES OF POTTING MATERIAL

1519

SOLVENT ANALYSIS OF STRESSES IN MOLDED POLYCARBONATE PARTS

1172

EVALUATION OF THERMOPLASTIC POLYESTER MOLDING RESINS

1189

EVALUATION OF POLYPHENYLENE SULFIDE

1260(REV.)

EVALUATION OF TPX MOLOING RESIN

1301 (REV.)

MOLOING CHARACTERISTICS OF THERMOPLASTIC FOAMS 
AUTHOR

HALTEF, C. L.

HALTER, C.L.

WALTERBACH, F. R.

WALTERS, J.A.

WATK INS, G. F.

HIEGAND, C. J.

WILEY, T. A.

WILLERTON, V. L.

WILLIAMS, G. A.

WILL IAMS, J. H.

WILLIAMS, J. H.

WILLYARD, D. L.

WILSON, B. G.

WILSON, J. L.

WILSON, J. L.

WILSON, M. L.

HILSON, M. L.

HILSON, M. L.

WINKLER, $G$. D.

WI SCHMAN, K. $B$.

WITTE, D. R.

WOLFE, L. A.

HONNELL, L. D.

WONNELL, L. $D$.

WONNELL, L. D.

MONNELL, L. D.

WONNELL, L. D.

WONNELL, L. D.

YOUNG, D. A.

YOUNGBERG, D. A.

YOUNGBERG, D. A.

YOUNGBERG, D. A.

ZAWICKI, L. R.

IAHICKI, L. R.
TItLe

INVESTIGATION OF NEW ENGINEERING THERMOPLASTICS

INVESTIGATION OF METHYLPENTENE POLYMER (TPX) MOLOING RESIN

ADVANTAGES OF GLASS MICRO-BALLOONS AS FILLER MATERIAL

COMPUTER AIDED TEST SYSTEMS

HOT GAS MOLDING OF POLYSTYRENE FOAM

STRUCTURAL STUDIES OF RIGID URETHANE FOAMS

EARLY PRODUCTION HISTORY ON HYBRIO MICROCIRCUITS CONTAINING BEATLLEAO OEVICES

ZIPPEREO MANORELS

ELECTRICAL CONTACT SURFACE TREATMENT

COVER EVALLATION fOR LARGE hYBRID microciRCUITS

HERMETIC SEALING OF HYBAIO MICROCIRCUITS-INITIAL CAPABILITY OEVELOPMENT

CHARACTER ILATION AND SCHEOULE DEVELOPMENT FOR GOLD-RIBBON HYBR IO-MICROCIRCUIT CROSSOVERS AUTOMATIC JESTING AND HANOLING, Qf, beAM LEAD TRANS ISTORS AND
DIODES

SANDIA/BENOIX STANDARD PROCESS CAPABILITY STUDY OF PRINTED HIRING BOARDS

FABRICATION OF MULTILAYER PRINTED WIRING BOARDS

FABR ICATION OF MULTILAYER PRINTEO MTRING BOARDS

PHOTOTOOL FABR ICATION METHOOS

EXPOSURE STUOY FOR DRY-FILM PHOTORESISTS

TEST EOUTPMENT ACCURACY

a New potting material-eXpandable polystrrene bead foAm

1905

\section{6}

275

535

48

11 16(REV.)

99

\$02 (REV.)

1500(REV.)

1930

897

1081

1349

1657

382 (REV.)

1286

1469

76

1647

MICROPROCESSORS AS A TOOL IN DETERMINING SFERICS AND

2122

IMPROVEO PROCESS FOR IMPREGNATING HIGH VOLTAGE TRANSFORMERS

1650

A NEW MIDE-RANGED DIRECT CURRENT CALIBRATION SOURCE

LASER INTERFEROMETER SCALING COUNTER

703

LASER CENTERING DETECTOR

933

1419

INTEGRATING SPHERE PHOTODETECTOR FOR MEASUREMENT OF CONTINUOUS-WAVE AND PEAK LASER POWER

1719

MEASUREMENT OF PEAK OPTICAL PÖNER FROM LASER DIODES (CONFERENCE PAPER)

UNIVERSAL MONITOR FOR CO2 LASERS (CONFERENCE' PAPER)

1907

REMOVAL OF METHYLENE OIANILIME FROM CHEMICAL PLANT WASTEWATER

1981

ELEMENTARY RELATIONSHIPS OF WORKER EFFICIENCY TO THE MORK ENVIRONAENT

415

CMARACTER I ZATION OF THERMOCOMPRESSION BOMDIMG PARAMETERS FOR BEAH-LEAD BONDING

1175

1176

POSTMORTEM TESTS

1800

HAC-TO-FLATPACK ATTACHMENT

1809 
Appendix F

SUBJECT INDEX OF REPORTS RELEASED 
SUBJECT

ABRASIVES

ADHES IVE BONDING

ADHESIVE BONDING

ADHESIVE BONDING

ADHESIVES

ADHESIVES

ADHESIVES

A DHESIVES

A DHESIVES

ADHESIVES

ADHESIVES

ADHESIVES

ADHESIVES

ADHESIVES

ADHESIVES

ADHE SIVES

ANALYSIS

ANALYSIS

ANALYZERS

ANALYZERS

ATTENUATORS

BACKSCATTER

BLDS

BONDING

BORON

BORON

BRALING

BRAL ING

BRAZINC

BRAZING

- BURRS

BURRS
TITLE

THREE-BOOY ABRASIVE WEAR WITH SMALL SIZE DIAMOND ABRASIVES

SURfaCe PREPARATION OF PLASTICS FOR ADHESIVE BONDING

BONDING FLUOROHALOCARBONS TO COPPER FOR ELECTRICAL APPL ICATIONS

EFFECTS OF SURFACE PREPARATION ON BOND STRENGTHS OF MAGNE S IUM

CONDUCTIVE ADHESIVES-A SUBSTITUTE FOR SOLDER

TEACH YOUR ENGINEERS TO DESIGN BETTER WITH ADHESIVES

GENERAL TECHNOLOGY OF ADHESIVES

DESIGNING AOHESIVES AMO ADHESIVE INTERFACES FOR OPTIMUM ELECTRICAL CONDUCTIVITY

THE BLENDING OF SEVEN LOTS OF MERGIMMMIDE L-425 FOR USE IN THE M300 ADHES IVE

THE BEgGS MMD TEST FOR DETERMINING THE SHELF IIFE OF FILM ADHESIVE

CHARACTERILATION OF FILM ADHESIVES

PREPARATION OF URETHANE ADHESIVES

CURE ANALYSIS OF AN ADHESIVE PRIMER

CHEMICAL, THERMAL, AND DYNAMIC MECHANICAL PROPERTIES OF FIVE ADHES IVES

THE TIME-TEMPERATURE CORRESPONOENCE OF THE DYMAMIC MECHANICAL PROPERTIES OF HALTHANE

SHELF-LIFE DETERMINATION OF EPOXY PREPREGS AND FILM ADHESI VES

DIGITAL ANALYSIS OF EBM BURST MAVEFORMS

X-RAY POLE ANALYSIS

DEVELOPMENT OF A COMPARATIVE ANALYZER FOR MI CROPROCESSOR

BARREL TUMBL ING IS IT ECONOMICAL?

CHARACTERIZATION OF THIN FILM MICROSTRIP ATTENUATORS AT MICROWAVE FREQUENCIES

DEVELOPMENT OF BETA BACKSCATTER PROBES USING HIGH-INTENSITY SOURCES (CONFERENCE PAPER)

CARRIER SYSTEM FOR TESTING AND_CONDITIONIMG OF BEAM LEAD. 1708 DEVICES

1

OPTIMIZATION OF LEAD FRAME BOND PARAMETERS FOR PRODUCTION OF RELIABLE THERMOCOMPR ESSI ON BONOS

BORIC ACIO ANALYSIS

ISOTROPIC ANAL YSIS OF EMRICHED BORON OY CHEMICAL IONIZATION MASS SPECTROSC OPY

PARALLEL GAP BRAZING

JOINING MAGNETIC ALLOY 49FE-49CO-2V TO 304L STAINLESS STEEL

BRALING TITANI UM STRUCTURES

CERAMIC-TO-METAL SEALING

BURRS PROOUCED BY TURNING

CANADA'S 100 MILLION DOLLAR THORMIN CAMADIAN MACHINERY AND ME TAL WORK I NG
668

1829

2060

2067

DOCUMENT

NUMBER

BOX-613-

2108

12

42

68

7

25

35

118

405

507

528

1549(REV.)

1656

1853

1867

2110

1857

1903

1969

1601

1940

140

936

1666(REV.)

1683 (REV.)

1748

1888 
SUBJECT

BURRS

BURRS

BURRS

BURRS

BURRS

CABLES

CABLES

CABLES

CAL IBRATION

CALI BRATION

CARBONIZATION

CASTING

CAST ING

CASTING

CASTING

CASTING

CHEMICAL ANALYSIS

CHEMICAL ANALYSIS

CHEMICAL ANALYSIS

CHEMICAL ANALYSIS

CHEMICAL VAPOR DEPOSITION CHEMICAL VAPOR OEPOSITION CHEMICAL VAPOR DEPOSITION CHEMICAL VAPOR DEPOSITION CHEMICAL VAPOR DEPOSITION CHEMICAL VAPOR DEPOSITION

CIRCUIT INTERCONNECTIONS

CIRCUIT INTERCONAECTIONS

CIRCUIT INTERCONNECTIONS

CIRCUIT INTERCONNECTIONS

CIRCUIT INTERCONNECT IONS

CIRCUIT STRESS

CIRCUITS

CLEANING
TITLE

NEEOED RESEARCH ON BURRS, DEQURRING, AND EDGE FINISHING ITO BE PUBLISHED BY SMEI

VIBRATORY DEBURRIMG: AN ANALYSIS OF COSTS IMACHINE TOOL AND BLUE BOOK)

MECHAN IZEO MEC HANICAL DEBURRING

DOCUMENT

NUMBER

$B 0 X-613-$

1891

1985

2017

DEBURR ING AN ANNOTATED BIBLIOGRAPHY, VOL $V$

2112

ELECTRCCHEMICAL DEBURRING ANOTHER SHARP SHORD IN THE BATTLE WITH BURRS

2132

1685

CABLES

HIGH DENSITY BRAIO CABLE SHIELOS

2037

RTV TOOLING EVALUATION

2038

PRECISION OC VOLTAGE REFERENCE

1746

RING-SHAPED BETA SOURCE ImPRONES GAGE ACCURACY

1966

CARBON FOAM-A CELLULAR STRUCTURAL MATERIAL FOR HIGH TEMPERATURE AP PLICATION

112

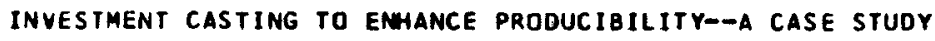

291

THE CREATION OF LARGE CASTINGS BY THE INVESTMENT PROCESS

COST SAVING THROUGH APPLICATION OF THE INVESTMENT CASTING PROCESS

CASTING MARPAGE STUDY

824(REV.)

1126 (REV.)

1368

REDUCE THE COST OF ELECTRONIC PACKAGING MITH INVESTMENT CASTINGS (CONFERENCE PAPER)

1720

THE ANALYSIS OF TIN-IRIOLUM-ZINC ALLOYS

37

COULOMETRIC DETERMINATION OF INORGANIC HAL IDE IMPURITIES IN POLYSTYRENE AFTER DISSOLUTION IN 2-GUTANONE

MOLECULAR CHARACTERIZLTION OF SILICONES BY GEL PERMEATION CHROMATOGR APHY

IMPROVED OXYGEN ANALYSIS USING A THALLIUM ACID PHTHALATE (TAP) CRYSTAL (CONFERENCE PAPER)

120

121

1622

ELECTROPOL ISHING CVD TUNGSTEN

242

CHEMICAL VAPOR DEPOSITION PROCESS EQUIPMENT

269

CVO SCALING STUDY

ELECTROPOL ISHING OF CHEMICAL VAPOR OEPOSITED TUNGSTEN

422

CHEMICAL VAPOR DEPOSIITION OF ALUMINUM

866(REV.)

FLOW ANO EXHAUST SYSTEM FOR CHEMICAL VAPOR DEPOSITION OF TUNGSTEN-RHENI UN ALLOY

1520

HOW TO REPAIR CONMECTOR TRI-LOCK PINS

96

ELECTRICAL CONNECTION STUDY

204

MINIATURE- INTERCONNECTION PROCESSING

1069(REV.)

PROCESSING OF MINIATURE INTERCONNECTIONS

1193 (REV.)

MINIATURE INTERCONNECTION DEVELOPMENT

1651 (REV.)

MEASURING STRESS IN LEAD WIRES

1775

FABRICATION OF MULTILAYER PRINTED MIRING BOARDS

1657 SOLUBILITY PARAMETER TECMNOLOGY ANO EVAPORAT IVE RATE
ANALYSIS USEO TO EVALUATE CONTAMINANTS 


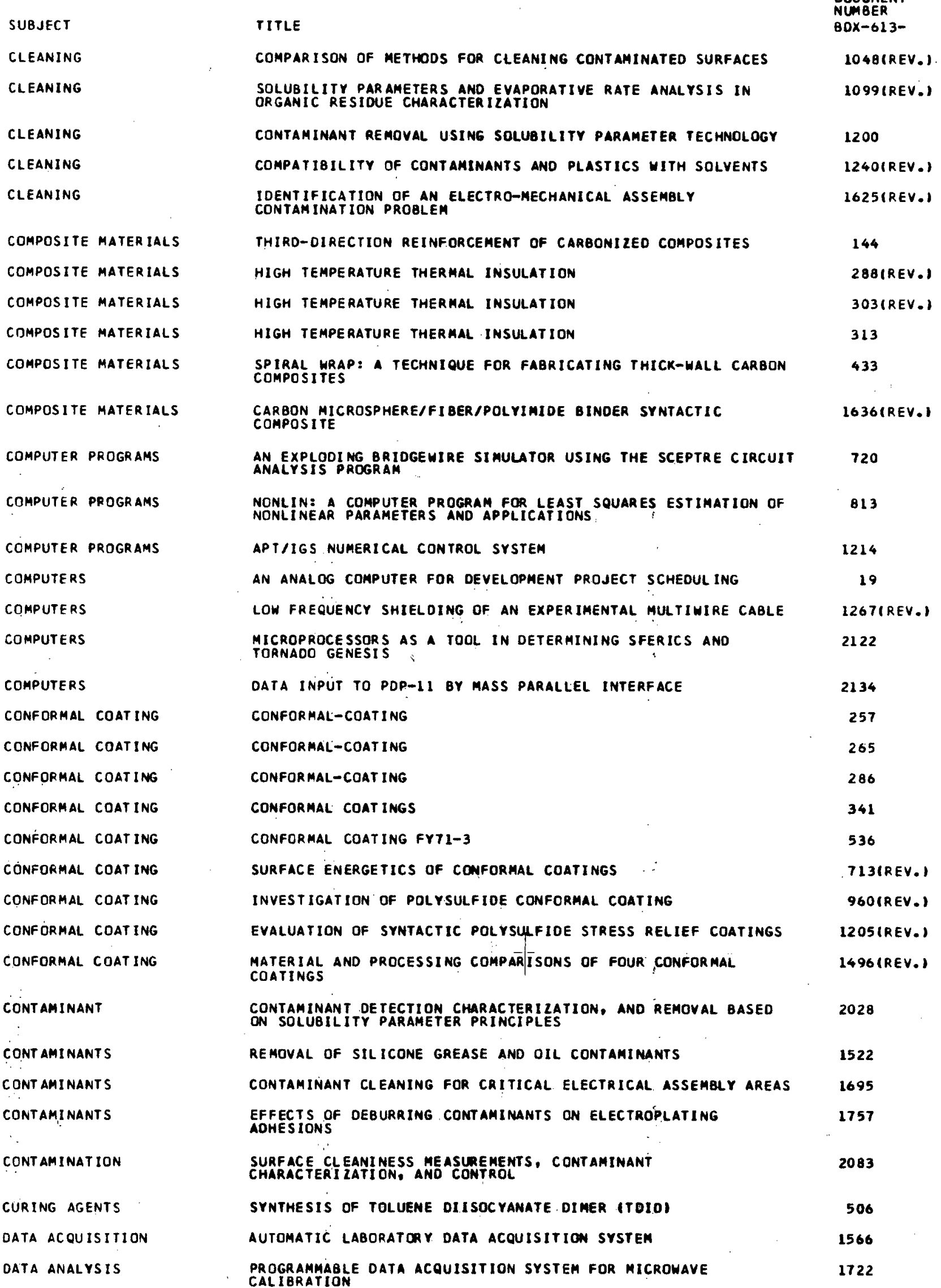


SUBJECT

DATA ANALYSIS

DATA ANALYSIS

DEBURR I NG

DEBURR I NG

OEBURR ING

DEQURR I NG

DEBURE I NG

DEBURR ING

DEBURR ING

DEBURR I NG

DEBURR I NG

OEBURP I NG

DEBURRING

DEBURF I NG

DEBURAING

DEBURR I NG

DEBURF I NG

DEBURR I NG

DEBURR ING

DEBURRING

DEBURR ING

DEBURRING

DEBURR ING

DEBURR ING

DEBURRING

DEBURR ING

DE BURR I NG

DEBURRING

DEBURR ING

DEBURR ING

DÉBURR I NG

DE BURR I NG

DEBURRING

DEBURRING

DEBURR ING

DEBURRING

DEBURR I NG

DEBURRING
TITLE

DIGITAL RLC ANALYSIS:OF CDU FIRESET $X$ UNITS ICONFERENCE PAPER I

REDESIGN OF BENOIX. VARIAGLES DATA SYSTEM (CONFERENCE PAPER)

RAOIUS GENERATION IN VI BRATORY OEBURRING

85

DUBURR ING: A B IBLIOGRAPHY

593

THERMAL SHOCK DEBLRRING

VIBRATORY DEBURRING

EXTRUDE HONE DEBURRING

meChanICAL DEBURRING OF PLASTICS

THE EFFECT OF CUTTING EDGE RADIUS ON POISSON BURR PROPERTIES

BRUSH DEBURRING OF hINIATURE PARTS

THE EFFECTS OF REAMING VARIABLES ON BURR PROPERTIES

PROPERTIES OF BURRS PROOUCED BY BALL BROACHING

THE BURR-- MANUFACTURING 'S PERENAIAL THORN

STATE-OF-THE-ART OF GENERAL DEBURR ING METHODS

THE MEASUREMENT OF BURRS

DEBURRING: AN ANNOTATEO BIBLIOGRAPHY

BURRS PRODUCED BY DRILLING

BURRS PROOUCEO BY SIDE-MILLING CUTTERS

BURRS PRODUCEO OY GRINDING

HANO DEBURRING OF PRECISION MINIATURE PARTS

EFFECTS OF DRILLING VARIABLES ON BURR PROPERTIES

642 (REV.)

735IREV.)

948

988 IREV.)

1047

1056 (REV.)

1083 (REV.)

1084 (REV.)

1112

1117

1120

1124

1248

1303 (FEV.)

1372

1443

1502

1503

1546

1559

1604

1605

1616

1620

1699

1703

1738

1751

1773

1777

1791

AN EXTENSION OF PROPOSED DEFINITIONS FOR BURRS AND RELATEO EDGE CONDI TIONS

THE BURR: A 1977 REPORT ON THE TECHNOLOGY OF REDUCING ITS

1793

AOVANCES IN DE BURRI NG

1977

HAND DEBURRING: A NECESSITY THAT CAN BE IMPROVED

1986 


\section{SUBJECT}

DEBURR. I NG

DEPOSITION

OEPOSITION

DESICCANTS

DESICCANTS

DESICCANTS

DESICCANTS

DETECTORS

DIISOCYANATES

DUBURR I NG

DYNAMIC TESTS

DYNAMIC TESTS

OYNAMIC TESTS

DYNAMIC TESTS

OYNAMIC TESTS

OYNAMIC TESTS

ECOLOGY

ELASTOMERS

ELASTOMERS

ELASTOMERS

ELASTOMERS

ELASTOMERS

ELASTOMERS

ELASTOMERS

ELASTOMERS

ELASTOMERS

ELASTOMERS

ELASTOMERS

ELASTOMERS

ELASTOMERS

ELASTOMERS

ELASTOMERS

ELASTOMERS
TITLE

hand deburring a plague dr the most economical mayt

DEPOSITION ANO CHARACTERILATION OF VACUUH-DEPOSITEO ALUMINUM FILM ON KAPTON LAMINATES

2100

1873

OEPOSITION OF ALUMINUA-COPPER ALLOY ON LAMINATEO POLYIMIOE OEPOSITION OF ALUNI NUA-COPPER ALLOY ON LAM
SUBSTRATES FROM AN RF INDUCTANCE SOURCE

1993

A STUOY OF THE DIMENSIONAL STABILITY OF MOLDED MOLECULAR SIEVE SUPPORT DESICCANT

LIQUID PROCESSING OF MOLDEO DESICCANT

380

ORY MIXING OF MOLOED DESICCANT

IMPROVED DENSITY CONTROL OF MOLOED OESICCANTS

FINAL REPORT ON MOTION SENSORS

PREPARATION AND PROPERTIES OF BLOCKEO DIISOCYANATES

PRECISION DEBURRING OF MINIATURE PARTS

THE DESIGN AND ADVANTAGES OF AN AIR-ACCELERATED IMPACT MECHANICAL SHOCK MACHINE

DYNAMIC TESTING OF EXPLODING BRIDGEMIRES

392 (REV.)

692 (REV.)

910(KEV.)

791

1796

1697

52

IMPACT TESTING OF MATERIALS USING AN EIGHT-INCH AIR GUN AND COMPUTER REOUCTION OF DATA

IMPACT TESTING OF FOAH-FILLEO PAPER HONEYCOMB WITH ENERGY-ABSORPTION CHARACTER ISTICS COMPARED TO. BALSA AND REDNOOO

TECHNIQUE FOR IMPACT TESTING OF CONFINED RIGID FOAM

1583 (REV.)

DYNAMIC RE SPONSE OF RIgID POLYURETHANE FOAM

1987

70

A NEW OEVELOPMENT. IN POL YURETHANE ELASTOMER PROCESSING WHICH REDUCES CRACK SUSCEPTIBILITY ANO BUABLE ENTRAPHENT

PROCESSING OF THIN CELLULAR SILICONE SLABS

353 (REV.)

STRETCH EFFECTS ON LOAD DEFLECTION ANO THICKNESS OF Y-3333 CELLULAR SILICONE SLABS

360(REV.)

COMPRESSION PLATENS FOR LOAD VERSUS DEFLECTION TESTING OF SILICONE ELASTOMERS

A STUDY OF THE SHELF AGING CHARACTERISTICS OF Y-3333 CELLULAR SILICONE

MOISTURE CONTENT STUDY OF Y-3333 CELLULAR SILICONE

769

944 (REV.)

946(REV.)

DEVELOPMENT OF ELASTOMER CUSHIONS

38T(REV.)

A STUOY OF THE COMPRESSION SET CHARACTERISTICS OF CELLULAR SILICDNE SUBJECTED TO ABNORMALLY HIGH STRAIN

388

A STUDY OF THE FUNCTIONAL CAPABILITIES OF AN AIR-BEARING COMPRESSION TEST FIXTURE

A STUDY OF tHE FUNCTIONAL CAPABILITIES OF a tOTAL AREA LOAOING COMPRESSION TEST F IXTURE

DEVEL OPMENT OF ELASTOMER CUSHIONS, FYT1-3

374

375 (REV.)

386 (REV.)

METREV.)

390 (REV.)

391 (REV.)

393 (REV.)

440(REV.)

447

458 (REV.)

493 (REV.)

1082 (REV.) 


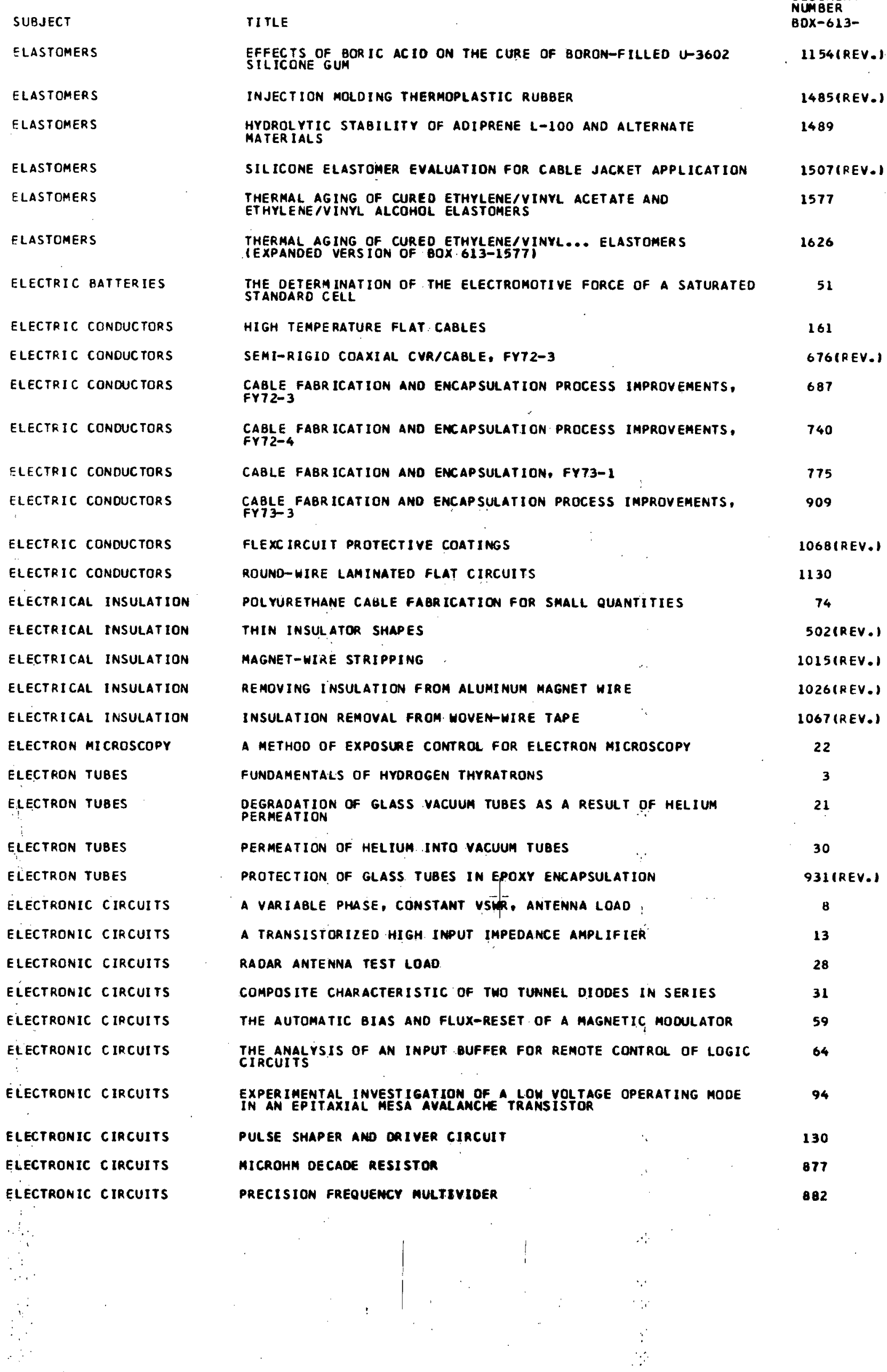


SUBJECT

ELECTRONIC CIRCUITS

ELECTRONIC CIRCUITS

ELECTRONIC CIRCUITS

ELECTRONIC CIRCUITS

ELECTRONIC TEST EQUIPMENT

ELECTRONIC TEST EQUIPMENT

ELECTRONIC TEST EQUIPMENT

ELECTRONIC TEST EOUIPMENT

ELECTRONIC TEST EQUIPMENT

ELECTRONIC TEST EQUIPMENT

ELECTRONIC TEST EQUIPMENT

ELECTRONIC TEST EQUIPMENT

ELECTRONIC TEST EQUIPMENT

ELECTRONIC TEST EQUIPMENT

ELECTRONIC TEST EQUIPMENT

ELECTRONIC TEST EQUIPMENT

ELECTRONIC TEST EQUIPMENT

ELECTRONIC TEST EQUIPMENT

ELECTRONIC TEST EQUIPMENT

ELECTRONIC TEST EQUIPMENT

ELECTRONIC TEST EQUIPMENT

ELECTRONIC TEST EQUIPMENT

ELECTRONIC TEST EQUIPMENT

ELECTRONIC TEST EQUIPMENT

ELECTRONIC TEST EQUIPMENTT

ELECTRONIC TEST EQUIPMENT

ELECTRONIC TEST EQUIPMENT

ELECTRONIC TEST EQUIPMENT

ELECTRONICS

ELECTROPLATING

ENCAP SULAT ING

ETCHANTS

EXTRUSIONS

FAILURE ANALYSIS

FILAMENT WINDING

FILAMENT WINDING
TITLE

CIRCUITRY FOR A HUMIOITY-LEVEL DETECTOR

A SOLIO STATE CIRCUIT BREAKER

TEN-GIT SAMPLE ANO hOLO OR PEAK hOLD CONYERTER

DUAL CHANNEL MIXER

TIMESAVING WITH a Parallel analyzing Chanmel attachment

RESISTANCE MEA SUREMENTS BY RATIO EMPLOYING VOLTAGE TO FREOUENCY CONVERTERS

a single pulse peak reading voltmeter

a SLOW TIME BASE READOUT SYSTEM FOR NON-REPETITIVE PULSE ANALYSIS

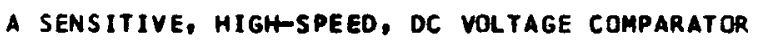

TEST EOUIPMENT ACCURACY

design of a variable phase, constant vsmr, micromave load

A NEW WIOE-RANGED DIRECT CURRENT CALIBRATION SOURCE

COMPUTER AIDED TEST SYSTEMS

OPTOELECTRONIC MEASUREMENT

EVALUATION OF METHODS FOR COMPUTER-AIDEO DESIGN OF TEST EQUTPMENT

A OIGITAL FREQUENCY MEASURING SYSTEM FOR PULSED RF DEVICES

LIGHT EMITTING DIODE testing and analYsis

A METHOD OF IMPROVING AMalog comparator Calibration ACCURACY

aUtomatic peak pQWER MEASUREMENT OF SHORT RF PULSES

READ-DNLY MEMORY IMPLEMENTATION STUDY

AUTOMATIC RADAR-SENSITIVITY MEASUREMENT

AUTOMATED TESTING IN A HIGH MOISE ENVIRONHENT

AN AUTOMATIC RF-METHORK ANALYZER AS A SUPPORT TOOL FOR ENGINEERING AND MANUFACTURING

COMPUTER AIOED FAILURE ANALYSIS

DIGITAL RECORDING ANO ANALYSIS OF FAST EBW BURST WAVEFORMS

SOlio STATE CONNECTOR

DOCUMENT

NUMBER

BOX-613-

1019

1062

1066

1282

34

63

67

71

72

76

79

134

275

276

349

167

956

2013

2046

1053(REV.)

1134 (REV.)

1138

1297 (REV.)

1369(REV.)

1381 (REV.)

1479

MULTIPURPOSE LABORATORY TEST SYSTEM APPLYING CAMAC

STANDARDS (CONFERENCE PAPER)

1717

1719

MEASUREMENT OF PEAK OPTICAL POMER FROM LASER DIODES (CONFERENCE PAPER)

PROCESS EFFECTS ON HIGH VOLTAGE ELECTRICAL ASSEMBL IES

1887

FEASIBILITY OF ELECTROPLATED GOLD FOR HYBRID MICROCIRCUITS

1740

DEVELOPMENT OF AN AUTOMATED ENCAPSULATION SYSTEM

1493

CHEMICAL CMARACTERIZATION OF A POLYPHOSPHORIC ACID ETCHANT

2078

MECHANICAL AND MICROSTRUCTURAL PROPERTIES CHARACTERIZATION OF HEART-TREATED BETA EXTRUDED TI-6AL-6V-2SN

1812

COMPUTER' AIDED FAILURE AMLLYSIS

2006

HELICAL TAPE WIHDER

607 (REV.)

FILAMENT WINDIMG INITIAL DEVELOPMENT
667 
SUBJECT

F ILAMENT WINDING

FOAM

FOAM

FOAM

FOAM

FOAM

FOAMS

FOAMS

FOAMS

FOAMS

FOAMS

GENERAL PROGRESS

GENERAL PROGRESS

GENERAL PROGRESS

GENERAL PROGRESS

GENERAL PROGRESS

GENERAL PROGRESS

GOLD-COPPER ANALYSIS

GRINDING, SLOTS

GROUP TECHNOLOGY

HEAT TREATMENT

HMCS

HOBBING

HONEYCOMB STRUCTURES

HONEYCOMB STRUCTURES

HONEYCOMB STRUCTURES

INSPECT ION

I NSPECTION

INSPECT ION

INSPECTION

INSPECT ION

INSPECT ION

INSPECT ION

I NSPECT ION

INSPECT ION

INSPECT ION

INSPECTION
TITLE

FILAMENT HINDING A THIN-MALL FIBERgLASS hEMISPHERE HIGH TEMPERATURE SYNTACTIC FOAM $\quad 1534$

GLASS MICROBUBBLE COMPOSITE FOAM 1596

DE VELOPMENT OF AN AUTOMATEO FOAN PROCESSING SYSTEM 1691

SYNTACTIC FOAM FROM SILVER COATEO MICROBUBBLES 1790

DENSITY MEASUREMENTS OF CURVED FOAM PARTS USING 1823

RADIOGRAPHIC-F ILM TECHMIQUE

MATER IAL EVALUATION OF POLYURETHANE FOAM, $0.05 \mathrm{G} / \mathrm{CM} 3$ DENSITY

CHARACTERIZATION OF LOW DENSITY RIGIO URETHANE FOAM 1890

EVALUATION OF CURE CYCLES FOR SYNTACTIC FOAM 1923

IMPROVED BONDABILITY OF MOLDED RIGIO URETHANE FOAM BY 1915 PLASMA TREATMENT (CONFERENCE PAPER)

POLYURETHANE FOAM $0.056 / C M 3$ OENSITY 1964

MECHANICAL PRODUCTS QUARTERLY 316

ELECTRONICS ENGINEERING QUARTERLY

QUALITY DIVISION QUARTERLY 404

ELECTRONIC ENGINEERING QUARTERLY 431

MATERIALS ENGINEERING QUARTERLY

PLASTIC PROOUCTS ENGINEERING QUARTERLY 480

DETERMINATION OF GOLD-COPPER ALLOY COMPOSITION BY X-RAY 1716 FLUORESCENCE ANALYSIS

SLOTIING BY ELECTROCHEMICAL GRINOING

1908

OBSERYATIONS ON IMPLEMENTING GROUP IECHNOLOGY FOR PRECISION 2045

MEAT TREATMENT OF GAL-4V TITANIUM FORGINGS TO OBTAIN HIGH

872 YIELD STRENGTH

MERMETIC SEALING OF HYBRIO MICROCIRCUITS-INITIAL CAPABILITY DEVELOPMENT

HOBBING RATCHET WHEELS FOR MINATURE MECHANISMS 1927

FILLED PAPER HONEYCOMB $\quad 190$

CARBON/CARBON MONEYCOMB $\quad 335$

MOOIFIED PAPER HONEYCOMB AS AM ENERGY ABSORBER 981 (REV.)

audare-A device for recording Data 10

MODERN GAGE CONTROL 39

A SPECIMEN TO FILM GAUGe FOR THE BACK REFLECTION CAMERA 46

ELONGATION AS A QUANTITATIVE MEASUREMENT 53

PRODUCTION TESTING OF MAGNETIC CORE SHIFT REGISTER ELEMENTS .62

A TECHNIQUE FOR MINIMIZING SLOTTED LINE MEASUREMENT ERROR 86

ULTRASONICS AS A THICKNESS MEASURING TOOL 88

SURFACE TEXTURE-MEASUREMENT OR CONFUSION : 99

SMALL ANGLE GEMERATOR 111

SHORT CIRCUIT DETECTION WITH LIQUID CRYSTALS 131

BORON SHOT GRADING AND INSPECTING 
SUBJECT

INSPECTION

INSPECT ION

INSPECTION

INSPECT ION

INSPECT ION

INSPECTION

INSPECTION

INSPECTION

INSPECT ION

INSPECT ION

INSPECT ION

INSPECT ION

INSPECTION

INSPECT ION

INSPECT ION

INSPECT ION

INSPECT ION

INSPECT ION

I NSPECT ION

INSPECTION

INSPECT ION

INSPECT ION

INSPECT ION

INSPECT ION

INSPECT ION

INSPECTION

INSPECTION

I NSPECT ION

INSPECT ION

INSPECT ION

INSPECTION

INSPECT ION

INSPECT ION

INSPECT ION

INSPECT IDN
TITLE

COMPARISON OF ORTHOGOMAL CURVILINEAR COOROINATE SYSTEMS FOR MINIMUA ERROR

INSPECTION OF FINE-PITCHED GEARS

FAST RECOVERY TIME DIODE TESTING

HIGH FREQUENCY PARAMETER TECHNIQUES

MEASUREMENT METHOD FOR POLYSTYRENE

LINING OF COMPRESSION SET TEST FIXTURES

VISUAL AND ELECTRICAL INSPECTION DF MINIATURE RADARS

RAPIO VISUAL SCANNING SYSTEMS FOR PCB'S, FYTI-2

RAPIO VISUAL SCANNING SYSTEMS FOR PCB'S, FY72-1

PHOTOGRAMMETRIC GAGING

NONDESTRUCTIVE TESTING OF PERCUSSIVE ARC WELDS

INSPECTION OF FINE-PITCHED GEARS

TESTING FOR MATERIAL FLAHS ANO THERMAL DESIGN DEFECTS USING HOLOGRAPHIC INTERFEROMETRY

DE TERMINATION OF HOLE LOCATION ON PRINTEO CIRCUIT BOARDS

TRANSDUCER FOR GAGING PRESSURE-SENSITIVE PARTS

OMNI-DIRECTIONAL ILLUMINATION FOR PRINTEO CIRCUIT BOARO INSPECTION

HOLOGRAPHIC INTERFEROMETRY

A HETHOO OF INSPECTING DIELECTRIC MATERIALS AT MICROMAVE FREQUENCIES

BETA BACKSCATTER MEASUREMENTS OF ALUMINUM COATIMGS ON MYLAR SUB STRATES

SPATIAL OISTRIBUTION FROM SMALL BETA SOURCES AND THE EFFECTS OF APERTURE SILE

AUTOMATED VISUAL INSPECTION SYSTEM FOR PRINTED WIRING ASSEMBLIES

PHOTOMULTIPLIER TUBE TESTIN: AND ANALYSIS

DEFECT DETECTION IN HIGH DENSITY BRAIDEO SHIELOS

REFLECTED LIGHT INTENSITY DISTRIBUTIONS FROM DEFECTS ON HI GHLY REF LECT IVE SPHERES

RAPIO VISUAL SCANNING

NONDESTRUCTIVE TESTING OF WELDED ELECTRICAL CONNECTORS

APPLICATION OF MOIRE. TECHNIQUES TO FIXTURELESS GAGING

CALIBRATION METHOD FOR COOROINATE MEASURING MACHINES

THREAD CERTIFICATION

an eVALUATION OF THE X-RAY fludRescence METHOD OF PREClOUS METAL PLATING THICKNESS MEASUREMENTS

MEASUR ING PENETRATION DEPTH OF ELECTRON BEAM WELDS

DOCUMENT

NUMBER

BDX-613-

184

309

315

347

355

410

451

527

538

547

$561($ REV.)

563 (REV.)

812

814

B79(REV.)

942

967 IREV.)

991

1028(REV.)

1061

1076IREV.I

1086

1122 (REV.)

1174 (REV.)

1187

1190(REV.)

1213

1242

1244 (REV.)

1284

1353

THE ANGULAR DI STRIBUTION OF DIFFUSE LIGHT REFLECTED FROA ISOLATED DEFECTS ON SUPERFINISHED SPHERES

1363 (REV.)

1401(REV.)

1497

OPTICAL SYSTEM FOR AUTOMATEO INSPECTION OF HIGHLY

REFLECTIVE SPHERICAL SURFACES.

FACTORS AFFECT ING VISUAL IMSPECTION OF THIM FILM NETWORKS 
SUBJECT

INSPECT ION

INSPECTION

INVENTORY CONTROL

INVENTORY CONTROL

LAMINATES

LAMINATES

LAMINATES

LAMINATES

LAMINATES

LAMINATES

LAMINATES

LAMINATION

LAPPING

LAPPING ABRASIVES

LASER CUTTING

LASER HEL DING

LASERS

L.IGHT

LUBP ICANTS

LUBRICANTS

MACHINING

MACHINING

MACHININGG

MACHINING

MACHINING

MACHINING

MACHINING

MACHINING

MACHINING

MACHINING

MACHINING

MACHINING

MACHIN ING

MACHINING

MACHINING

MACHINING

MACHINING
TITLE

FAST NEUTRON GAUGING FEASIBILITY STUOY

SEMIAUTOMATIC VISUAL INSPECTION SYSTEM FOR THIN-FILM

1770 NE THORKS

1813

ECONOMIC I APROVEMENT OF INVENTORY SAMPLING

885

WORK-IN-PROCESS INVENTORY

1145

COMPRESSION MOLOING OF LAMINATES

327

AUTOCLAVE FABR ICATION TECHNIQUES FOR FLAT CABLES

579 (REV.)

DEVELCPMENT OF A SELF-CONTAINED LANIMATING FIXTURE

927 (REV.)

STEEL-RULE diE CONTOURING EVALUATION

1049

DEVELOPMENT OF A FORMING STATION

1123 (REV.)

EFFECTS OF THERMAL EXPANSION ON EPOXY GLASS LAMINATES

1171

hIGH TEMPERATURE NONP LAMAR Laminates

1783

TESTING THE IN TERLAMINATE adHESION OF mULTILAYER PRINTED MIRING BOARDS

LAPPING EQUIPMENT INSTRUMENTATION $\quad 1264$

ALUMINA LAPPING ABRASIVE STUDY 1692

LASER CUTTING OF REVLAR LAMIMATES 1877

FINITE ELEMENT ANALYSIS OF LASER INOUCEDTHERMAL SHOCK 2044

UNIVERSAL MONITOR FOR CO2 LASERS ICONFERENCE PAPERI 1907

FRAUNHOFER DIFFRACTION PATTERNS FROM APERTURES ILLUMINATEO 2016 WITH NONPARALLEL LIGHT

LIGHT LOAO FRICTION COEFFICIENT ANO MEAR STUDY OF THREE BONOED SOLID LUBRICANTS

1170

FRICTION ANDWEAR STUDIES FOR BONDED THINFILM, LUBRICANTS

1316

RADIUS GENERATION IN VIBRATING FINISHING 90

A MICRODRILL LAPPING MACHINE

102

DRILLING PRINTEO CIRCUIT BOARDS

279

CONFIRMATION OF A NAJET ORILLING TECHNIQUE FOR SMALL HOLES 306

FINE-EOGE BLANKING AND PIERCING $\quad \ldots \quad 366$

AFT SUPPORT MACHINEO FROM TITANIUM

ULTRAPRECISION TOQ GRINDING 552

MACHINING MINIATURE SLOTS ANDEILLETS. $\quad \ldots \ldots t 6$

BIBLIOGRAPHY: MACHINING MINIATURE PARTS AND MINIATURE 585 FEATURES

OPTIMIZING MINIATURE CYLINORICAL PIECEPART MACHINING

TO8IREV.) CAPABILITIES

LASER BALANCING OF VERGE AND SHAFT ASSEMBLIES

724

LASER REMOVAL OF DIELECTRIC from fLAT CABLES

82 O(REV.)

HDLE FINISH CONTROL IN 4330 VAMAOIUN MODIFIED STEEL

863

REMOVAL OF INSULATION FROM MULTICONOUCTOR WIRE TAPE USING A CARBON DIOXIDE LASER

887(REV.)

SHAPING RATCHET TEETH

973

PROFILE MILLING

980(REV.)

GRINDING MINIATURE PIMION geARS 
SUBJECT

MACHINING

- MACHINING

MACHINING

MACHINING

MACHINING

MACHINING

MACHINING

MACHIN ING

MACHINING

MDA

MEASUR ING

MEASUF. ING

MEASUR ING INSTRUMENTS

MEASUR ING INSTRUMENTS

MEASUR ING INSTRUMENTS

MEASUR ING INSTRUMENTS

MEASUR ING INSTRUMENTS

MEASUR ING INSTRUMENTS

MEASUR ING INSTRUMENTS

MEASUR ING INSTRUMENTS

MEASURING INSTRUMENTS

MEASURING INSTRUMENTS

MEASUR ING INSTRUMENTS

MECHANICAL PROPERTIES

METAL WORK ING

METAL WORKING

METAL WORKING

METAL WORKING

METAL WORKING

MICROCIRCUITS

MICROCIRCUITS

MICROCIRCUITS

MICROCIRCUITS

MICROELECTRONICS
TITLE

ELECTROCHEMICAL GRINOING

GEAR CUTTING

1159

1392

MINIATURE CORE ASSEMBLY FABRICATION AND TEST PROCESSES.

1417

SKIVING

1429

FIXTURING AND HANOLING OF MINIATURE COMPONENTS

1482

ECONOMIC OPTIMILATION TECHNIQUES APPLICABLE TO PRECISION MINIATURE MACHINING

machinaBILITY as RELAJEd TO PRECISION Miniature parts

1512 (REV.)

1567

machinability of metals as related to miniature precision COMPONENTS

1723

ELECTROCHEMICAL MACHINING DEEP THROUGH hOLES

1948

REMOYAL OF METHYLENE OIANILINE FROM CHEMICAL PLANT WASTEWATER

1981

EQUIPMENT FOR A LASER SLRFACE MEASURING SYSTEM

1848

USE OF A DISPLACEMENT MEASURING SYSTEM FOR POSITIONING A CONTOUR

2119

CONDUCTIVITY TESTING OR EDOY CURRENT TESTING WITH THE FH-1OOMAGNATEST CONDUCIIVITY METER

AUTOMATIC MEASURING OF TEMPERATURE COEFFICIENTS

INTERFEROMETER PRISM: POR 187

127

ROLAMITE SENSORS

697 (REV.)

LASER INTERFEROMETER SCALING COUNTER

703

DIFFERENTIAL PRESSURE MERCURY MANOMETER

878

LINEAR TRANSDUCER TIP PRESSURE CHECKER

904

LASER CENTERING DETECTOR

933

IMPROVED RADIO-FREQUENCY POHER STANDARDS

971

DEVELOPMENT OF A LIGHT LOAD FRICTION TESTER:

1104

INTEGRATING SPMERE PHOTODETECTOR FOR MEASUREMENT OF CONTINUOUS-WAVE ANO PEAK LASER POHER

1419

FraCtURE MECHANICS TESTING CAPABILITY

1531 (REV.)

FOCUS ING PRESSURE SHOCK WAVES IN LIQUIO FOR EFFICIENT HYDROELECTRIC FORMING

THE EFFECT OF HIGGH STRAIN-RATE|FORMING ON THE
RECRYSTALI LATION OF COPPER

THE PROPERTIES OF ALUMINUM AND COPPER AFTER

HIGH-ENERGY-RATE FORMING

102

DEEP DRAWN STAINLESS STEEL COVERS

868

ORBITAL HEADFORM STAKING APPLICATIONS FOR MINIATURE

EL ECTROMECHANICAL ASSEMBLIES

1582

DETERMINING THE MANUFACTURA

1779

WET FILM PHOTOLITHOGRAPHY OF HACS CONTAINING VIAS

1800

HMC-TO-FLA TPACK ATTACHMENT

1809

SEMICONDUCTOR DIE ATTACHMENT

129

AN EVALUATION OF SCATTERING PARAMETER TECHíI QUES AS APPLIED TO FIELDOEFFECT TRANSISTOR CHARACTERILATION 
SUBJECT

MICROELECTRONICS

MICROELECTRONICS

MICROELECTRONICS

MICROELECTRONICS

MICROELECTRONICS

MICROELECTRONICS

MICROELECTRONICS

MICROELECTRONICS

MICROEL ECTRONICS

MICROELECTRONICS

MICROELECTKONICS

MICROELECTRONICS

MICROELECTRONICS

MICROELECTRONICS

MICROELECTRONICS

MICROELECTRONICS

MICROELECTRONICS

MICROELECTRONICS

MICPOELECTRONICS

MICROEL ECTRONICS

MICROELECTRONICS

MICROELECTRONICS

MICROELECTRONICS

MICROEL ECTRONICS

MICROELECTRONICS

MICROELECTRONICS

MICROELECTRONICS

MICROELECTRONICS

MICROEL ECTRONICS

MICROELECTRONICS

MICROEL ECTRONICS

MICROELECTRONICS

MICROELECTRONICS MICR OELECTRONICS
TITLE

MICROBONDING AND PACKAGING FOR HYBRIO MICRDELECTRONIC CIRCUITS

BEAM LEAD SEMICONDUCTOR TESTING AND HANOLING

THICK-FILM NET WORKS

DETERMINATION OF SEMICONDUCTOR JUNCTION PROFILES BY ANGLE LAPPING OF SEMICONOUCTOR HAFERS

BEAM LEAD SEMICONDUCTOR TESTING AND FAILURE ANALYSIS

A SUSPENDEO METAL MASK FOR THICK-FILM PRINTIMG

EFFECTS OF GEOMETRY ON THICK-FILM RESISTORS

PHOTOMASK MEASURING SYSTEMS

OEPOSITION OF CHROMIUN BY RAOIO FREQUENCY SPUTTERING

PLATED-THR OUgh hOLES BY PLASMA-ENHANCED DEPOSITION

PHOTORESIST CHARACTER IZATION METHOD FOR HYBRIO MICROCIRCUITS

INPUT AND OUTPUT DEVICES FOR A COMPUTERIZED MASK-MAKING
SYSTEM

HYBRIC MICROCIRCUIT PHOTOLITHOGRAPHIC PROCESS

TRIMMABLE FILM RESISTIORS

THIN-FILM HYBRID MICROCIRCUIT SUBSTRATE METALLIZATION

BEAM LEAD DEVICES AND THE HMC MANUFACTURER

CLEANING AND ASSEMBLY TECHNIQUES, FY73-1 JUNCTION FIELD EFFECT TRANSISTOR DEGRADATION CAUSED BY
ELECTROSTATIC DISCHARGE

BONDING DEGRADATION IN THE TANTALUM NITRIDE-CHROMIUM-GOLD METALLILATION SYSTEM

STRENGTH MEASUREMENTS ON TRANSISTOR HEADER LEAD WELDS

CHARACTERIZATION ANO SCHEDULE DEVELOPMENT FOR GOLO-RIBBON HYBRID-MICROCIRCUIT CROSSOVERS

PANTOGRAPH POSITIONING SYSTEM FOR. INSPECTING MICROCIRCUITS

MASK GENERATION

BEAM LEAD TESTING, hANOLING, AND FALLURE ANALYSIS

STATIC CHARGE AND CURRENT LEAKAGE EFFECTS RELATING TO MANUFACTURE OF JFET ASSEMBLIES

SOLUTION CONTROL FOR HYGRIO MICROC IRCUIT ETCHING

LSI INTEGRATED CIRCUIT TEST DEVELOPMENT

HIGH TEMPERATURE REVERSE BIAS. ANO POHER BURN-IM AT TRANS ISTOR JUNCTION TEMPERATURES FROM 150 TO 300 DEG. $C$

CONTROL OF ELECTROSTATIC DAMAGE TO SOL ID STATE dEVICES

AUTOMATIC TESTING ANO HANDLING OF BEAM LEAD TRANSISTORS AND DIODES THE EFFECT OF RAPIDLY CHANGING VOLTAGES ON THICK-FILM
RESISTORS

CHARACTERI ZATION OF A CHROMIUMGOLD DEPOSITION PROCESS FOR THE PRODUC TION OF THIN FILA HYBRID MICROCIRCUITS

beAm LeAd deVICE ano hyBa ID microcircuit testing

DEVELOPMENT OF PROCESSES FOR HYARID MICROCIRCUITS CONTAINING BEAH-LEAD DEVICES

860
1113

194

363 (REV.)

604(REV.)

614

648

649

650

651

652

653

654

678

728

7608REV.1

779

793(REV.)

815

871

897

932

959(REV.)

975(REV.)

994 (REV.)

1012

1017

1038

1080

1081

1085(REV.)

1109

1115(REV.) 
SUBJECT

MICROELECTRONICS

MICROELECTRONICS

MICROELECTFONICS

MICR OELECTRONICS

MICROELECTRONICS

MICROELECTPONICS

MICRDELECTRONICS

MICRDELECTRONICS

MICROELECTRONICS

MICROELECTRONICS

MICROELECTRONICS

MICROELECTRONICS

MICROELECTRONICS

MICROELECTRONICS

MICROEL ECTRONICS

MICROELECTRONICS

MICROELECTRONICS

MICROELECTRONICS

MICROELECTRONICS

MICROELECTRONICS

MICROELECTRONICS

MICROELECTRONICS

MICROELECTRONICS

MICROELECTRONICS

MICROELECTRONICS

MICROELECTRONICS

MICROELECTRONICS

MICROEL ECTRONICS

MICROELECTRONICS

MICROEL ECTRONICS

MICROELECTRONICS

MODELING
TITLE

EARLY PROOUCTION HISTORY ON HYBRID MICROCIRCUITS CONTAINING BEAH-LEAO DEVICES SPRAY PHOTORESIST DEVELOPMENT ON MULTIPLE HYBRID
MICROCIRCUIT SUBSTRATES

MASS ANODIZATION DF TANTALUM-NITRIOE RESISTORS

A RAPIO TCR TEST METHOD FOR TANTALUM-NITRIDE RESISTIVE FILM

CHARACTER ILATION OF A CHROME-GOLD THIN FILM DEPOSITION PROCESS QY AUGER ANALYSIS

CHARACTER I ZATI ON OF THERMOCOMPRESSION BONDING PARAMETERS FOR BEAH-LEAD BONDING

1116(REV.)

1148

1155 (REV.)

$1167($ REV.)

1173

1175

REMOVAL OF HYBRID MICROCIRCUIT BEAM-LEAD DEVICES FOR POSTMURTEM TESTS

1176

AN ACTIVE LASER TRIMMER FOR PRODUCTION TRIMMING OF FUNCTIONAL HYBRID MICROCIRCUITS

1182

INFRARED BAKING OF POSITIVE PHOTORESIST FOR HYBRID MICROC IRCUIT FABRICATION

1246

HYBR IO MICROCIRCUIT INTRACONNECTION PROCESSES

1257

beAM LEAO dEVICE TEMPERATURE TESTING

1275

PHOTORESIST APPLICATION BY RQLER COATING

1288

INTERCONNECTION TECHNOLOGY

MEMORY ASSEMBLY TO HYBRIO MICROCIRCUITS

1346

1371 IREV.)

MULTIPLE HYBRIO MICRDCIRCUIT PROCESSING

1383

1456

FAILURE MODES OF BEAM LEAD SEMICONOUCTORS IN THIN FILM HYBR ID MICROCIRCUITS (CONFERENCE PAPER)

1461 HAND PUNCH
SUB STRATES

99.5 PERCENT' ALUMINA

FACE-UP BDNDING OF BEAM LEAD DEVICES

1462(REV.)

automatic lead frame plating

SURFACE EFFECTS ON SEMICONDUCTORS

1464

1468

1477

ELEMENTS AT MI CROMAVE FREQUENCIES

ANALYSIS OF THICK FILM CONDUCTOR INKS

1491

COVER EVALUATION FOR LARGE MYBRID MICROCIRCUITS

1500 (REV.)

WIGGLY PHASE SHIFTERS AND OIRECTIONAL COUPLERS FOR RAOIO FREOUENCY HYBR IO MICROC IRCUIT APPL. ICATIONS ICONFERENCE PAPER :

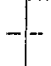

MANUFACTUR ING PROCESSES FOR HYBRID MICROCIRCUITS CONTAINING 1524

1532

PRODUCTION COATING OF VIAS IN ALUMINA SUBSTRATES WITH VACUUA EVAPORATED CHROMIUM AND GOLD (CONFERENCE PAPER)

1535

HANOL ING AUTOMATION AND TEMPERATURE CONTROL ADDED TO TRANSISTOR S- PARAMETER MEASUREMENT (CONFERENCE PAPERI

1536

LASER CONTOURED MO DRILLEO SUBSTRATES FOR THICK FILM APPLICATIONS

1563(REV.)

1564 (REV.)

CHARACTERIZATION OF CHROMIUM-GOLD FILMS FOR HYBRID MICROC IRCUITS

1575

PLATING hYQRIO miCROCIRCUITS

2020

COMPUTER APPLICATIONS ANO HYBRIO MICROCIRCUITS

1950 
SUBJECT

MOLD HEAT ING

MOLO HEAT ING

MOLDING

MOLDING

MOLOING

MOLDING

MOLDING RESINS

MOLOING TECHNI QUES

MOLDING TECHNIQUES

MOLOING TECHNIQUES

MOLDING TECHNI QUES

MOLDING TECHNI QUES

MOLOING TECHNIQUES

MOLOING TECHNIQUES

MOLOING TECHNIQUES

MOLDS

NORO CORE

NETWORK ANAL YZER

NUMERICAL CONTROL

NUMERICAL CONTROL

NUMERICAL CONTROL

NUMERICAL CONTROL

NUMERICAL CONTROL

NUMERICAL CONTRQ

NUMERICAL CONTROL

PHOT OENGRAVING

PHOTOENGRAVING

PHOTOENGRAVING

PHOT OENGRAVING

olASMA

PLASTICs, CELLULAR

PLASTICS, Cellular

Plastics, cellular

PLASTICS, CelluLar

PLASTICS, CelluLar
TITLE

COMPUTER SIMULATION OF THE MOLD. HEATING ANO CONTROL LOOP, USING CONVENTIONAL CONTROL STRATEGIES

MOLD HEATING ANO COOL ING SYSTEMS

TRANSFER MOLDING OF CLOSE-TOLERANCE PARTS

CHEMICAL ANO PHYSICAL CMARACTERIZATION OF A KEVLAR-FILLEO EPOXY MOLDING COMPOUNO

MOLD HEATING AND COOL ING SYSTEMS

DIGITAL CONTROLLER DESIGN, AMMLYSIS, AND IMPLEMENTATION FOR THE MOLO MEATING AND COOLING SYSTEM

INVESTIGATION OF METHYLPENTENE POLYMER (TPX) MOLDING RESIN

PRESSURE AND TEMPERATURE GRADIENTS IN TRANSFER MOLDING OF ELECTRONIC ASSEMBLIES

THEORY AND OPERATION OF THE BENDIX MOLO HEATING AND CODL ING EQUIPMENT

fluid flon analysis in the molo heating and COOLING loOp

EXPER IMENTAL TESTING FOR MOLO TEMPERATURE RESPONSES

moloING MINIATURE PLASTIIC PARTS

DESIGN IMPROVEMENTS FOR CYCLIC INJECTION MOLDS

EVALUATION OF LOOP AND PARALLEL OIL CHANMEL DESIGNS

WEAR PREVENTION IN MOLOS USED TO MOLD BORON-FILLED ELASTOMERS (CONFERENCE PAPER)

COOLING ALUMINUM molos USING HEAT PIPES

1764

1990

1542

1816

1979

1980

1905

333

369

742

IIIIREV.)

1212 (REV.)

1239

1423

1684

2039

fabrication ano performance of a laminateo nonvolatile NONDESTRUC TIVE READOUT MEMORY CORE

1763

A MOVING HEAD DISC OPERATING SYSTEM FOR AN 8542 A AUTOMATIC NETHORK ANALYZER (CONFERENCE P.APER)

1652

ONC COMMITTEE FINAL REPORT

376

DIRECT NUMERICAL CONTROL OF MACHINE TOOLS,

NUMER ICALL Y-CONTROLLED ASSEMBLY

540(REV.)

804

PROTOTYPE OIRECT MUMERICAL CONTROL SYSTEM

1585

EDITING CAPABILITY FOR DIRECT NUMERICAL CONTROL

1688

OVERVIEH OF DIRECT NUMERICAL CONTROL

1917

DIRECT MUMERICAL CONTROL AT BENOIX KANSAS CITY DIVISION

EFFECT OF SODI UM HYDROXIDE ON_ALUMINUM FOIL THICKNESS

IMPROVEO CURVED VACUUM BLANKET'S FOR PHOTORESIST EXPOSURE

ALUMINUM ETCHANT CONTROL STUDY

2018

SLSREV.)

953

955

1105(REV.)

ALUMINUM ETCHANT STUDIES

2030

GAS PLASMA EFFECTS ON PRINTED CIRCUIT BDARD MATERIALS

16 OF FLEXIBLE URETHANE FOAMS

RIGIO URETHAME FOAMS FROM E-CAPROLACTONE POL YESTERS

38

48

STRUCTURAL STUDIES OF RIGID URETHANE FOAMS

69

THERMAL CHARACTERISTICS OF SOME RIGIO URETHANE FOAMS

75 
SUBJECT

PLASTICS, CELlular

PLASTICS, CELlULAR

plastics, cellular

PLASTICS, CELLULAR

PLASTICS, CELLULAR

PLASTICS, CELLULAR

PLASTICS, CELlular

PLASTICS, CELLULAR

PLASTICS, CELLULAR

PLASTICS, CELLULAR

PLASTICS, CELLULAR

Plastics, Cellular

PLASTICS, CELLULAR

PLASTICS, CELLULAR

PLASTICS, CELLULAR

PLASTICS, CElluLAR

plastics, cellular

Plastics, cellular

PLASTICS, CELLULAR

PLASTICS, CEllular

plastics, cellular

plastics, cellular

PLASTICS, CELLULAR

PLASTICS, CELLULAR

PLASTICS, CELLULAR

PLASTICS, CELLULAR

PLASTICS, CELLULAR

PLASTICS, CELlular

PLASTICS, CELLULAR

plastics, cellular

PLASTICS, CELLULAR

plastics, cellular

plastics, cellular

plastics, Cellular
TITLE

STRUCTURAL APPLICATION CHARACTERISTICS OF A RIGID URETHANE FOAM SYSTEM

PHYSICAL/Chemical PRopentIES OF RIgIO URETHANE FOAM 106

CHARACTERIZATION OF AN EPOXY FDAM 125

THERMAL PROPERTIES OF RIGID URETHANE FOAMS 132

CONTINUOUS FOAM BILLET .FABRICATION

EFFECTS OF AMINE CATALYSTS ON THE PHYSICAL PROPERTIES OF A 170

RIGID URETHANE FOAM SYSTEM

SYNTACTIC FOAM EVALUATION 177

DETERMINATION OF FOAM DENSITY 180

RIGIO EPOXY FOAM ENCAPSULATION

MEDIUM DENSITY MOLDED FOAM

A NEW TECHNIQUE FOR DETERMINING GAS DIFFUSIVITY IN HETEROGENEOUS MEDIA--PLASTIC FOAM

FILLEO foAm MANUFACTUR ING AND TESTING TECHNI QUES

SYNTACTIC FOAM, FYTO-3

POL YURETHANE FOAM MATERIAL AND PROCESS DEVEL OPMENT

THERMAL PROPERTIES OF LACTONE ESTER-BASEO RIGID URETHANE FOAMS

THE SYNTHESIS OF A LOH-DENSITY RIGID EPOXY FOAM

MATERI AL AND PROCESS DE VELOPMENT OF POLYURETHANE FOAM, FYT1-4

AM EVALUATION OF COMMERCIAL polvaryl polyisocranates IN A BENDIX KANSAS CITY FOAM SYSTEM

EVALUATION OF FOAM MIXING MACHINES

A STUOY OF POL YETHER-POLYOL-AND POLYESTER-POLYOL-BASED

RIGIO URETHANE FOAM SYSTEMS

EFFECT OF VOIDS ON THE COMPRESSIVE STRENGTH OF MOLDEO RIGIO URETHANE FOAM

HOT GAS MOLDING DF POLYSTYRENE FOAM

MIXING HEAO FOR URETMANE FOAM METERING AMD MIXING MACHINES

MATER IAL AND PROCESS DEVELOPMENT OF POL YURETHANE FOAM, FY72-1

mechanical and physical pRoperties of oRganic foAm

IN-LOT VAR IATIONS IN POLYSTYRẼNe EXPANDABLE beads aND BEAD FOAM

VOLATILE LOSS IN POLYSTIRENE FOAM

FABRICATION OF POLYIMIDE SYNTACTIC FOAM PARTS

MATER IAL AND PROCESS DEVELOPMENT OF POLYURETHANE FOAM, $\underset{\text { FYT }}{\text { MATER } 3}$

MATER IAL AND PROCESS DEVELOPMENT OF POL YURETHANE FOAM, FY73-1

FABRICATION OF OPEN CELL POLYETHYLEME FOAM

EFFECT OF AMINE CATALYST ON COMPRESSIVE PRÓPERTIES OF URE THANE FOAM

LOW DENSITY FOAMS FOR VACUUM INSULATION

RISE-RATE TESTING OF URETHAME FOAMS
869

938

195(REV.)

209

229

252

253

300

406

407

485IREV.I

508

522

53

532

535

537

553(REV.)

562 (REV.)

564

578

582 (REV.)

718

797

870

941 
SUBJECT

plastics, cellular

PLASTICS, CELLULAR

plastics, cellular

PLASTICS, CELLULAR

PLASTICS, CELLULAR

PLASTICS, CELLULAR

PLASTICS, CELLULAR

plastics, cellular

PLASTICS, CELLULAR

PLASTICS, CELLULAR

plastics, cellular

plastics, cellular

plastics, cellular

PLASTICS, CELLULAR

PLASTICS, FILLED

PLASTICS, MOLDING

PLASTICS, MOLDING

plastics, molding

PLASTICS, MOLDING

PLASTICS, MOLOING

PLASTICS, MOLDING

PLASTICS, MOLDING

PLASTICS, MOLDING

PLASTICS, MOLDING

PLASTICS, REINFORCED

PLASTICS, REINFORCED

PLASTICS, REINFOR CED

PLASTICS, REINFORCEO

PLASTICS, REINFORCED

PLASTICS, REINFORCED

PLASTICS, THERMOPLASTIC

plastics, thermoplastic

PLASTICS, THERMOPLASTIC

PLASTICS, THERMOPLASTIC
TITLE

DESIGN MANUAL FOR POLYSTYRENE FOAM, RELATING PHYSICAL, MECHANICAL, AND CHEMICAL PROPERTIES

STATIC COMPRESSIVE TESTING OF RIGID POLYURETHANE FDAM

PREDICTING COMPRESSIVE STRENGTHS IN URETHANE FOAMS AT HIGH STRAINS

POL YURETHANE/IMIDE MODIFIED FOAM

1110

EFFECTS OF VARYing FORMULATIONS OF THREE TDI POLYURETHANE FOAMS

RIGID URETHANE FOAM BASEO ON METHYL-ALPHA-(D)-GLUCOSIDE AND E-CAPRCLACTONE

EVALUATION OF A HIGH TEMPERATURE SYNTACTIC FOAM

HIGHDENSITY POLYSTYRENE GEAD PREPARATION

FEASIBILITY STUDY OF PROOUCTION OF 0.24 G/CM3-DENSITY POLYSTYRENE BEAD FOAM

FLEXIBLE POL YURETHANE FOAM

THERMALLY CONDUCTIVE SYNTACTIC FOAM

LOW DENSITY EPOXY ENCAPSULANTS

MOLDING CHARACTERISTICS OF THERMOPLASTIC FOAMS

FEASIBILITY STUDY FOR PRODUCING HIGHDENSITY POLYSTYRENE BEAD FOAM

EFFECTS OF TEMPERATURE ON FILLEO EPOXY ENCAP SULATION MATERIALS

TRANSFER MOLOING ClOSE TOLERANCE PARTS

TRANSFER MOLDING OF CLOSE TOLERANCE PARTS

AN INVESTIGATION OF INTERNAL CAVITY PRESSURE FOR INJEC TION-MOLDED POLYCARBONATES

MOISTURE EFFECTS ON TMERMOSET MOLDING COMPOUNDS

EFFECTS OF MOISTURE UPON SPIRAL FLOW IN THERMOSET MOLOING COMPOUNDS

PRESSURE OISTR IBUTION IN POLYCARBONATE INJECTION MOLDINGS

AN INVESTIGATION OF INTERNAL CAVITY PRESSURE AND THICKNESS RESPONSE FOR INJECTION- MOLDED POL YCARBONATES

EVALUATION OF TPX MOLOING RESIN

DOCUMENT

NUABER

BDX-613-

1033

1036 (REV.)

1103(REV.)

1137 (REV))

1140 IREV.

1178 (KEV.)

1299 (REV.)

1298 (REV.)

1345 (REV.)

1409 (REV.)

1411 (REV.)

14 L4 (REV.)

1422 (REV.)

11

166

283

601 IREV.I

821 IREV.)

930 (REV.)

951

1002

1301 (REV.)

EFFECTS OF MOI STURE AND TRANSFER PRESSURE ON SURFACE FINISH AND SHRINKAGE OF 52-20-30 DIALIYL PHTHALATE

OESIGN OF JOINTS FOR FILAMENT-NOUNO CYLINDERS

1569

DESIGN AND DEVELOPMENT OF A THREE-DIMENSIONAL INTERLOCKED, FILAMENT-MOUND COMPOSITE

SPLIT FIBERGLASS HOLDER

AFAP REINFORCED COMPOSITE CASE (FYTO-3)

REINFORCED COMPOSITE CASE, FYTO-4

OIELECTRIC ANALYSIS OF AN EPOXY PREIMPREGMATED MATERIAL

testing PRoblems of sPaCe age plastics

THERMOPLASTIC FRONTIER LAMS-GOOO AND BAD GUNS

142

143

198

254

289 IREV.I

1396

58

60

DESIGN OF AN INSULATOR WHICH MUST SURVIVE HIGH G-FORCES

213

DESIGN EVALUATION OF INSULATOR PIN

294 
SUBJECT

PLASTICS, THERMOPLASTIC

PLASTICS, THERMOPLASTIC

PLASTICS, THERMOPLASTIC

PLASTICS, THERMOPLASTIC

olastics, thermoplastic

PLASTICS, THERMOPLASTIC

plastics, thermosetTING

PLASTICS, THERMOSETTING

PLASTICS, THERMOSETTING

PLASTICS, THERMOSETTING

PLASTICS, THERMOSETTING

PLASTICS, THERMOSETTING

PLASTICS, THERMOSETTING

PLASTICS, THERMOSETTING

PLASTICS, THERMOSETTING

PLASTICS, THERMOSETTING

PLASTICS, THERMOSETTING

PLASTICS, THERMOSETTING

PLATING

Plating

PLAT ING

PLATING

PLAT ING

plating

PLAT ING

plating

PLATI ING

Plat ING

PLATING

PLATING

PLATING

PLATING

Plating

Plating

PLATING

Plating
TITLE

STRESS-CRACXING AMD FRACTURE TOUGHNESS OF POLYCARBONATE MATER IAL

LOW TEMPERATURE PROPERTIES OF GENERAL ELECTRIC LEXAN 8070-112 THIN POLYCARBONATE FILM

SOL VENT ANALYSIS OF STRESSES IN MDLDED POLYC ARBONATE PARTS

EVALUATION OF THERMOPLASTIC POLYESTER MOLOING RESINS

evaluation of polyphenYlene sulfioe

INVESTIGATION OF NEW ENGINEERING THERMOPLASTICS

CAST FLEXI BLE URETHAME POLYMERS

AN EPOXY RESIN WITH IMPROVED ELECTRICAL PROPERTIES

A CAST FLEXIBLE ESTER-URETHANE POLYMER

BORGN TRIFLUORIDE-AMINE AOOUCTS AS MARDENERS FOR LIOUID EPOXY RESINS

EPON 828/MENTHANE DIAMINE CURING FACTORS

COMPUTERIZEO EXOTHERM CALCULATIONS FOR LARGE EPOXY CASTINGS

A DESCRIPTION OF VARIOUS METHODS OF PREPARING POLYAMIC ACIDS AND POLYIMIDES

EVALUATION OF HIGH TEMPERATURE DIELECTRICS :

CHARACTERI ZATION OF CONATHANE EN-4 URETHANE RESIN

LINEAR THERMAL EXPANSION OF FILLED EPOXY RESINS

PREPARATION OF A CTBN-MOOIFIEO EPOXY RESIN *

PREPARATION OF a REMOVABLE POL YURETHANE ENCAPSULANT

THE TARNISH RESISTANCE OF GOLD. PLATING OVER SILVER

ELECTROPLATING ON TITANIUH AND TITANIUM ALLOYS

PHYSICAL PROPERTIES OF ELECTROFORMEO NICKEL FROM SULFAMATE SOLUTIONS

GETTING MORE OUT DF PLATIMG THROUGH PROCESS SPECIFICATIONS

ELECTRODEPOSITION OF MICKEL-COBALT ALLOY: OPERATING PARAMETERS AND PHYSICAL PROPERTIES OF THE DEPOSITS

EVALUATION OF CARBONYL NICKEL

FEASIBILITY STUDY: PLATING THICKMESS GAGE

ELECTRODEPOSITION OF ALUMINUM

ELECTROFORMED GILOING METAL ROIATING BAND

POLYMERIC CO-DEPOSITION IN GOLD ELECTROPLATE

PROPERTIES OF PLASMA-SPRAYED ALUMINUM COATING

THE REFEREMCE ELECTRODE AND ITS USE IN DETERMINING INOIVIDUAL ELECTRODE POTENTIALS

ELECTROPLATING

BLECTR

ELECTROFORMED NICKEL MOLD INSERTS

AUTOMATED ANALYSIS OF HYPOPHOSPHITE: IN ELECTROLESS MICKEL SOLUTIONS

Plating UN IFORMITY

PROTECTIVE COATINGS FOR URANIUM-NIOBIUM ALLOYS

GOLD PLATIMG
500

615

DOCUMENT

NUMBER

$B O X-613-$

512

TOSIREV.1

1172

1189

1260(REV.)

1425(REV.)

20

41

65

105

109

114

509

S34IREV.I

842 (REV.)

952

1121 (REV.)

1406

24

32

61

66

77

104

115

236

310

408

412

430

454

499

805 
SUBJECT

Plating

PLAT ING

PLATING

PLATING

plating

PLATING

PLATING

Plating

PLATING

plat Ing

PLATING

Plating

PLATING

PLATING

plat ING

Plat ING STANDARdS

platinum

POLYETHYLENE

polystrRene

POLYSTYRENE FOAM

POTTING

POTTING

POTT ING

POTTING

POTTING

POTTING

POTTING

POTT ING

POTT ING

POTTING

POTTING

POTTING

POTT ING

PRE-SEALING

PRECISION MACHINING

PREC ISION MACHINING
TITLE

ALUAINUM PLATIMG GRAPHITE FIBERS AND BERYLLIUM WIRE

determining trace metals in gold deposits ano plating baths

ALUMINUM PLATING OF BeRYLIUM MIRE

ELECTRODEPOSITION OF ALUMINUM FROM ETHER-HYDRIDE SOLUTIONS

ELECTRODEPOSITING PT AND PT-IR ALLOYS FROM MOLTEN CYANIDES: CHEMICAL PROCESS STUDIES

plating on polyimide

THE FEASIBILITY OF ELECTROPLATING PLATIMUM-IRIDIUA OUT OF FUSED CHLORIDES

SURFACE PREPARATION OF KAPTON FOR ALURINUM DEPOSITION

DeVELOPMENT OF PLATING THICKNESS STANDARDS

multillayered plating thickness

PLATING THICKNESS StanoARDS

ELECTRICAL MEASUREMENTS DF PLATING THICKMESS

ELECTRICAL COMPENSATION SYSTEM FOR PLATED-THRQUGH- HOLE TESTER

ELECTRCPLATED GOLD FOR HYBRID MICROCIRCUITS

micaghm resistance measlatements to determine plating

THICKNESS ON PWB

DE YELOPMENT OF PLATING THICKNESS STAMDAROS FOR THE PHYSICAL VAPOR DEPOSITION OF ALUMINUM ON KAPTON

PLATINUM RESISTANCE THERMOMETER CALIBRATION ON THE INTERMATIONAL PRACTICAL TEMPERATURE SCALE OF 1968

PREPARATION AND PRDPERITES OF DEUTERATED POL YETHYLENE

a NeW POTTING material-eXPandable polystrrene bead goam

MECHANICAL PROPERTIES OF 0.2 G/CH3 POLYSTYRENE BEAO fOAM ADVANTAGES OF GLASS MICRO-BALLOONS AS FILLER MATERIAL

POTTING PROBLEMS RELATEO TO PACKAgING DESIGN

NEW POTENTIAL IN RIGID POTTING COMPOUNDS

TECHNIQUES IN USING POL VURETHANE MATERIALS FOR ENCAP SULATION

EXOTHERM LOCATION CAN CAUSE PROBLEMS IN POTTING

BONDING ST.RENGTH OF ENCAPSULATING MATERIALS TO HOOKUP HIRE

TEMPERATURE DEPENOENT PROPERT̄̄ ẼS OF POTTING MATERIALS

USE OF EN-7 TO ENCAPSULATE AMALYZER ASSEMBiLIES

COMPARISON OF ALTERMATE MATERIALS FOR MOCA CUREO ADIPRENE $L-100$

DESIGN MANUAL FOR POTTING ELECTRONIC ASSEMBLIES

TEMPERATURE DEPENDENT PROPERTIES OF POTTING MATERIAL

DEPOTTING ELECTRONIC ASSEMBLIES

IMPROVEO PROCESS FOR IMPREGMATING HIGH VOLTAGE TRANSFORMERS

REHABILITY DF PACKAGED. BEAM-LEAD TRANSISTORS BUILT MITH DIFFERENT PRE-SEAL ASSERBLY TECHNI QUES

DRILLIING MINATURE HOLES PART 1

DR ILL ING MIMATUAE hOLES, PART 2
124

1785

DOCUMENT

NUMBER

$B D X-613-$

86SIREV.S.

873

874

875IREV.)

1064(REV.)

IITTIREV.I

1227

1454(REV.)

1709

1795

1805

1942

1943

2114

2128

1634

1373

1570

1647

1725

6

23

56

123

135

1133(REV.I

1135 (REV.)

1207

1216(REV.)

1519

1530

1650

1952

1786 
SUBJECT

PRECISION MACHINING

PRECISION MACHINING

PRESSURE VESSELS

PRINTED CIRCUITS

PRINTED CIRCUITS

PRINTED CIRCUITS

PRINTED CIRCUITS

PRINTED CIRCUITS

PRINTEO CIRCUITS

PRINTED CIRCUITS

PRINTED CIRCUITS

PRINTED CIRCUITS

PRINTED CIRCUITS

PRINTED CIRCUITS

PRINTED CIRCUITS

PRINTED CIRCUITS

PRINTED CIRCUITS

PRINTED CIRCUITS

PRINTED CIRCUITS

PRODUCTION METHODS

PRODUCTION METHOOS

PROOUCTION METHOOS

PRODUCT ION METHODS

PRODUCT ION METHODS

PRODUCT ION METHOOS

PRODUCTION METHOOS

PROOUCT ION METHOOS

PRODUCT ION METHODS

PRODUCT ION METHODS

PRODUCTION METHODS PRODUCT ION METHODS PROOUCT ION METHOOS PROOUCT ION MET HOOS PRODUCT ION METHODS PROOUCTION METHOOS
TITLE

OR ILLING MINIATURE HOLES, PART 3

NUMBER

BOX-613-

MDORE NUMBER 5 DIAMOND TURNING MACHINE DES IGN ANO

1787 OE SCR IPT ION

FABRICATION OF FAILSAFE PRESSURE VESSELS

2051

STATE OF CURE OF PRINTEO CIRCUIT BOAROS

712

233

FABRICATION OF MULTILAYER PRINTEO WIRING BOARDS

382 (REV.)

496

505

SOLOER SPLATTER CAUSEO BY OUTGASSING HOLES IN PRINTEO WIRING BOAROS

PREVENTION OF TIN-LEAD OVERHAND IN PLATEO COPPER-CLAD PRINTED WIRING BOAROS

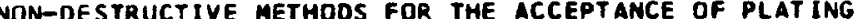
THICKNESS ON PRINTED CIRCUIT BDARDS

ACCURACY OF HEIGHT METHOO USED TO DETERMINE ALUMINUM FOIL THICKNESS

PLATED THROUGH-hOLES IN FLEXCIRCUITS

670

1044

1245 (REV.)

1254(REV.)

INPROVED PRECISION ALUMINUM ETCHANT

REWORK OF MULTILAYER PRINTEDHIRINGBOARD ASSEMBLIES

$1281($ REV.)

1315

SANDIA/BENOIX STANDARD PROCESS CAPABILITY STUDY OF PRINTED WIR ING BOARDS

ROLLER LAMINATION OF MEDIUM TEMPERATURE FLEX CIRCUITS

1349

1412(REV.)

ELECTRICAL CONDUCTIVITY STUDY OF ETCHEO-OUT PLATED-THROUGH HOLES IN DOUBLE-SIOED PRINTED WIRING BOARDS

1434

EXPOSURE STUDY FOR DRY-FILM PHOTORESISTS 1469

EVALUATION OF ALKALINE ETCHANTS FOR PRINTED WIRING BOARDS 1523

HMC SPRAY, DEVELOPING, STRIPPING, AND ETCHING 1803

PRODUCTION OF A RELAY UNDER CONTROLLED CONDITIONS 2

TOTAL PURCHASING DATA PROCESSING SYSTEM 17

Why nOt MEChanize maintenanCe $\quad \therefore \quad 29$

TOTAL PROCUREMENT SYSTEM PLAN 33

MANAGEMENT CONSIDERATIONS FOR CLEAN MORKING AREAS 44

A PLANT ENGINEERING : DISCIPLINE FOR CLEAN HORKING AREAS

BENEFITS ACCRUEO TO HIGHLY PRECISE MANUFACTURE FROM CLEAN WORK POSITIONS.

GRAPHIC AID TO DESIGN OF SPLICED QUARTER-MAVE RESONANT LINES

A COEFFICIENT OF THERMAL EXPANSION CAN BE DESIGNEO

ANSWERS TO LOH-COST PRODUCTION. PACKAgING

FLEXIBLE CABLE LACING

REPORT ON DEVELOPMENT OF IMDUCTANCE STANOARDS 98

ZIPPEREO MANORELS 99

FLUIOIZED BED COATING 100

SOLID LUBRICANT APPLICATION BY SPRAY ANO TUMBLE METHODS 108

DESIGNING AND MOLDING MODULE HOUSINGS FOR ELECTRONIC PACKAGES 
SUBJECT

PRODUCTION METHODS

PRODUCT ION METHODS

PRODUCTION METHODS

PRODUCTION METHOOS

PRODUCTION METHODS

PRODUCTION METHODS

DRODUCT ION METHODS

PROOUCT ION .METHOOS

PRODUCTION METHOOS

PROOUCTION METHOOS

PRODUCT ION MET HOOS

PRODUCTION METHODS

PRODUCT ION METHODS

PROOUCTION METHODS

PRODUCT ION METHOOS

PRODUCTION METHOOS

PRODUCT ION METHOOS

PRODUCT ION METHODS

PRODUCT ION METHODS

PRODUCT ION METHODS

PRODUCT ION METHOOS

PRODUCT ION METHODS

PRODUCT ION METHODS

PRODUCTION METHODS

PRODUCT ION METHODS

PRODUCTION METHODS

PRDOUCT ION METHODS

PROOUCT ION METHODS

PROOUCT ION METHODS

QUAL ITY CONTROL

QUALITY CONTROL

QUALITY CONTROL

QUALITY CONTROL

QUAL ITY CONTROL

QUALITY CONTROL
TITLE

DETERMINING PROPER THREAD ENGAGEMENT BY NOMOGRAPH

DESIGN CONSIDERATIONS IN THE PRODUCTION OF STRIPLINE FERRITE CIRCULATORS

maChINE COOLANT SELECTION and maintenance manual

BORON SHOT FABRICATION

the oetermination of average cycle time for an automated WAREHOUSE

ELEMENTARY RELATIONSHIPS OF WORKER EFFICIENCY TO THE WORK ENVIRONMENT

LINE OF BALANCE ACCURACY

139

146

205 (REV.)

227 (REV.)

274

415

442

COMPUTER SIMULATION MODELING OF AN AUTOMATEO HAREHOUSE SYSTEM

SCS CASE

PROCESSING EVALUATION OF MAGNETIC PRODUCTS

RUST PREVENTION IN TOOL STORAGE AREAS

NONMETALLIC TOOLING CAPABILITIES AND ECONOMICS, FY72-4

NONMETALLIC TOOL ING CAPABILITIES AND ECONOMICS, FY73-1

IMPROVEO WORK DIRECTORS

NONMETALLIC TOOLING CAPABILITIES ANO ECONOMICS

autOMATED SCHEDULING of MANUFACTUR ING RESOURCES

456

463

518 (REV.)

559

738

778

9641REV.1

1065IREV.)

1077

RESOURCES FORECASTING SYSTEM

1078

AUTOMATIC PHOTOMASK ALIGMHENT AMO EXPOSURE ON 3.75 BY 4.50 INCH SUBSTRATES

LOIIREV.I

AUTOMATIC MACHINE APPLICATION OF SOLIO FILM LUBRICANT

MAINTENANCE WORK STANDARDS AND COMPUTERIZED MORK SYSTEM

1169

1235

DEVELOPMENT OF AN IMPROVEO BONDING FIXTURE

1252 (REV.)

1285

1286

1319

A PRESS FIT INTERFERENCE-LINIT STUDY FOR MINIATURE ASSEMBLIES

PROCESS ACCEPTANCE AND AOJUSTMENT TECHNIQUES FOR SWISS AUTOMATIC SCREW MACHINE. PARTS

1354|REV.)

SMALL POWDER PARTS

GLASS SEALING PROCESS FOR ELECTFTICAL FEEOTHROUGHS

1386 (REV.)

1424(REV.)

GROUP TECHNOLOGY

1478

COMPUTER AIDEO FAILURE ANALYSIS

1552(REY.)

APPLICATION OF PUNCHED CARD TECHNIQUES FOR OPTIMIZING RELIABILITY

1

SEMICONDUC TOR FAILURE AMALYSIS USING THE CHARACTER ISTIC CURVE

COMPUTERIZEO QUALITY CONTROL

a standards and calibration mogram In a large defEnSE PLANT

WHAT CAN THE QUALITY ENGIMEER EXPECT FROM MEASURING INSTRUMENTS

COMPARATIVE DUCTILITY OF BRITTLE METALS BY A PRECISION ELONGATION TECHNIQUE 
SUBJECT

QUALITY CONTROL

QUALITY CONTROL

QUALITY CONTROL

QUALITY CONTROL

QUALITY CONTROL

QUALITY CONTROL

QUALITY CCNTROL

QUALITY CONTROL

OUALITY CONTROL

QUALITY CONTROL

QUALITY CONTROL

QUAL ITY CONTROL

QUALITY CONTROL

QUALITY CONTROL

QUALITY CONTROL

RADIATION TESTS

RADIATION TESTS

RELAYS

RESINS

RESINS

SILICONES

SINTER ING

SOLDER

SOLDER

SOLDER ALLOYS

SOLDER I NG

SOLDERING

SOLDERING

SOLDER ING

SOLDER ING

SOLDERING

SOLDER ING

SOLOERING

SOLDER ING

SOLOER ING
TITLE

DIAGNOSIS OF MALFUNCTIONS IN COMPLEX ELECTRONIC ASSEMBLIES ELECTRICAL CONTACT SURFACE TREATMENT

QUALIFICATION OF MINIATURE INTERNAL THREADS USING AIR. FLOW INVESTIGATION OF KNITTED FABRICS

RECOMMENDED SCAEW TORQUES FOR $0.6,0.8$. AND 1.0 UNIFIED NATIONAL MINIATURE THREADS

DEL IQUESCENT-CHEMICAL MAXIMUM hUMIOITY INDICATOR

YIBRATION ANALYSIS

APPLICATION OF STATISTICAL METHODS TO MANUFACTURING OPERATIONS

DETECTION AND SUPPRESSION OF TRANSISTOR OSCILLATION DURING POWER-BURN-IN

MAXIMUM HUMIOITY INOICATOR

EFFECTS OF COATING AND CLEANING ON COROMA AND HIGH-VOLTAGE BREAKDCWN IN ELECTRONIC ASSEMBLIIES

LASER STRIPPING FOR WIRE BREAKAGE DIAGNOSIS

DETECTION AND CONTROL OF POWER-LINE TRANSIENTS AFFECTING TESTER AND GAGE PERFORMANCE

EVALUATION OF MIGH DENSITY BRAID TERMINATION FATIGUE LIFE AND CONDITION

FEASIBILITY STUOY OF OIDOE PRECONDITIONING :

QUANTITATIVE AUGER ELECTRON ANALYSIS OF GOLD-COPPER ALLOYS

MULTILAYERED PLATING THICKNESS MEASUREMENT

DIGITILING CONTACT BOUNCE.

SYNTHESIS OF POLYAMINOBISMALEIDE

337 (REV.)

402 (REV.)

716(REV.)

783

825

921

926

1079

1144(REV.)

1150IREV.I

1181 (REV.)

1208(REV.)

1421

1435 (REV.)

1465(REV.)

1466 (REV.)

1632 (REV.)

1756

1480

1988

1880

45

EFFECTS OF SINTERING ATMSPHERES ON THE ELECTRICAL ANO CERAMIC PROPERTIES OF LEAO ZIRCONATE-LEAO TITANATE FERROELECTRICS

SOLDERABILITY TEST DEVELOPMENT

1706

A BOUNOARY VALUE DETERMIMATION OF CONTACT ANGLE 2003

RDOM TEMPERATURE AGING PROPERTIES OF SOME SOFT SOLDER ALLOYS

SOLDERING TO GOLO PLATING

1599

SOLOERABILITY TESTING

PREVENTING FAILURES IN SOLOERING TERMINALS :

LEAD WIRE SOLOERABILITY. TEST

28

SOLDERS FOR THICK GOLD PLATING

133

FLATPACK REFLON SOLDERIMG

141

INFRARED REFLOH SOL DERING

169

REQUIREMENTS FOR REFLOW SOLDERING OF INTEGRATEO CIRCUIT

187

SOLOERABIL ITY PROBLEMS CAUSED BY GOLO ELECTROPLATE

324

FLATPACK INSTALLATION BY SINGLE-PROBE REFLON SOLDERING 


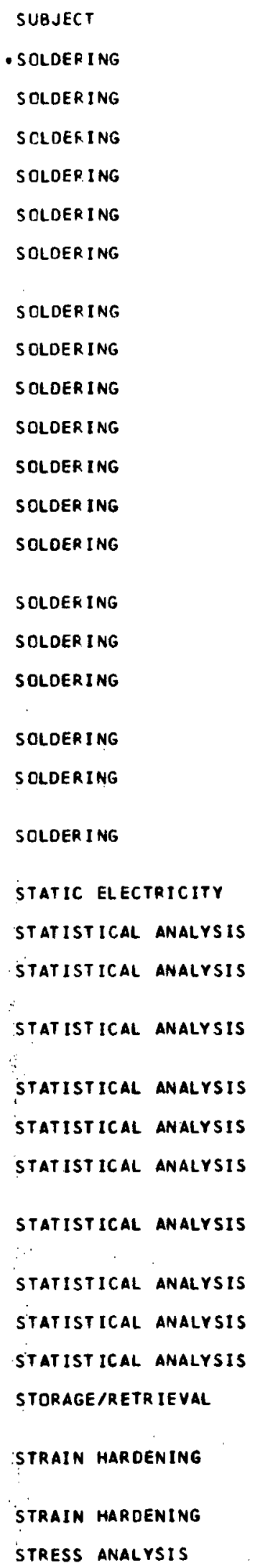

TITLE

FLOW SOLDERING ANO SUPPORTING PROCESSES 330

SOLDER LEVELING OF PRINTED WIRING BOAROS 443

SOLDER LEVEL ING APPLIEO TO MANUFACTURING 465(REV.)

HAND TOOL FOR INSTALL ING SOLOER FORMS OVER COMPONENT LEADS ' 602 (REV.)

SOLDER LEVELING ' 616

REQUIREMENTS FOR REFLOW SOLDERING OF INTEGRATEO CIRCUIT 664 FLATPACKS

SOLDER LEVELING

FLATPACK SOLDERING TO SOLDER-LEVELED PRINTED WIRING BOARDS 839 (REV.)

ULTRASONIC TINNING OF COMPONENT LEAOS 996

SOLDER SLEEVES

FEASIBILITY OF DRAG SOLDERING

COA725 TRANSFORMER LEAO SOLOER JOINT EVALUATION

EQUIPMENT EVALUATIONS AND PROCESS TECHNIQUES FOR DRAG SOLDERING PRINTED WIRING ASSEMBLIES

INOUCTION SOLDERING EVALUATION

1042 (REV.)

1051 (REV.)

1234 (REV.)

1404 (R.EV.)

LOH TEMPERATURE ALUMINUM SOLDERING ANALYSIS

1453

1557

STRESS REL IEF TECHNIQUES FOR REOUCING THERMAL FATIGUE IN 1655 SOLDER JOINTS

SOLDERING AUTOMATION

1865

QUANTITATI VE COMPARISON OF ELECTRONIC COMPGNENT-SOLDER 1868

JOINT STRESS RELIEF IN ENCAPSULATED ASSEMBLIES

TEMPERATURE VERSUS TIME CURVES For MANUAL ANO AUTOMATED SOLDER ING PROCESSES

STATIC ELIMINATION WORK STATIONS

1920

BINOMIAL SAMPLING PLANS INDEXED BY AQL AND ITPD

1953

ONE SIDED TOLERANCE LIMITS OF A NORMAL OISTR IBUTION BASED

47

ON SAMPLE MEAN AND MEAN RANGE

49

CONFI DENCE LIMITS ON A PROPORTION DEFECTIVE CHARACTER IZED

COMPUTERILED INTERPRETATION OF A OATA CHARACTERISTIC B7

CHARACTERISTICS OF SKIP LOT SAMPLING 116

SOLUTIONS FOR THE MAGR SEQUENTIAL T-TEST, SEQUENTIAL 117

VARIABLES SAMP LING TO CONTROL_THE PERCENT DEFECTIVE.

QUANTITATIVE ADJUSTMENTS FOR PERCENT DEFECTIVES IN 510 NON-NORMAL DISTRIBUTIONS

CCNTINUOUS STRATIFIED SAMPLING OF IN-PROCESS INVENTORY 1032

SAMPLE SILE TO SET SPECIFICATION LIMITS 1223

APPLICATION AREA FOR STATISTICAL METHODS (CONFERENCE PAPER) 1600

SIMULATION MODELING OF AN AUTOMATEO MATERIAL 1870

STORAGE/RETRIEVAL SYSTEM

THE STRENG THEN ING OF STEEL BY MARSTRAINING MITH 103 HIGH-ENERGY-RATE FORMIMG EQUIPMENT

STRAIN HARDENING EXPONENT FOR 17-4 PH STAINLESS STEEL. 577

A OISTORTION ENERGY FALWRE THEORY FOR ORTHOTROPIC 136 MATERIALS 


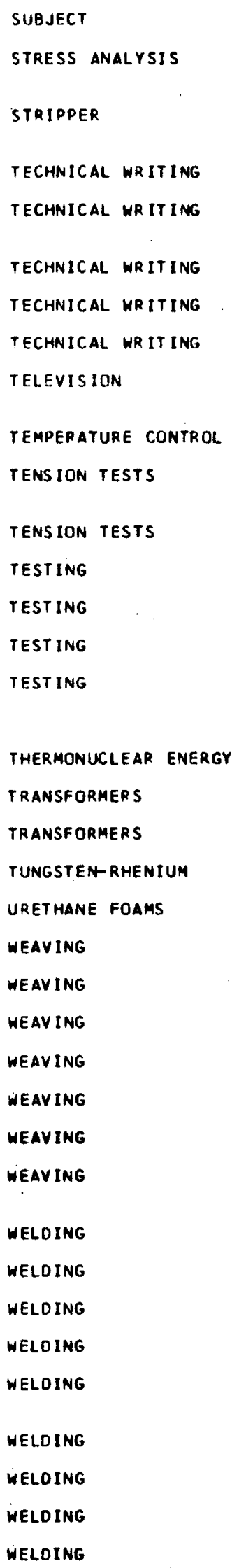

TITLE

A STRESS ANALYSIS OF THICK-MALL ORTHOTROPIC SPHERICAL VESSELS

N-METHYL-2-PYRROLIOONE AS A STRIPPER FOR PLASTIC POTTING COMPOUNDS

WR ITING METAL FINISHING PROCESS SPECIFICATIONS

WRITERS GUIOE TO THE PREPARATION OF ARTHORK FOR TECHNICAL REPORTS

THE EDITOR'S NOTEBOQK

TYPIST'S gUIDE TO BENDIX KANSAS CITY TECHNICAL REPORTS

THE INCOMPLEAT STYLIST

MONITORING SINUSOIDAL VIBRATION INVIRONMENTS WITH A TV SYSTEM

DIGITAL CONTROLLER FOR CYCLIC TEMPERATURE CONTROL A METHOD FOR INTERRUPTING A HIGH STRAIN RATE TENSION TEST
BEFORE SPECIMEN FRACTURE

TENSILE TESTING OF HIGH STRENGTH MATERIALS

1955

TELEMETRY TEST METHODS

1811

CONTACTOR SPEEDS S-PARAMETER TESTS

1967

mass PARALlel INTERFACE INPUTS OATA TO POP-11

1968

DESTRUCTIVE TESTING OF HYBRID MICROCIRCUITS CONTAINING THERMOCOPRESSION BONDED DEVICES ANO LEAD-INOIUM SOLDERED CAPACI TORS

2081

SORTING AND HANOLING LASER TARGETS

1325

the impedance of a coll placed on a conducting plane 27

FERRITE-CORE TRANSF ORMER BONDING AND POTTING

992

DEVELOPMENT OF A METALLOGRAPHIC METHOD FOR TUNGSTEN-RHENIUM

1744

HIGH LOAD BEARING FLEXIBLE URETHANE FOAMS

1750

HIGH-DENSITY BRAID CABLE SHIELD, FYT2-1

543 (REV.)

HIGH-DENSITY BRAID CABLE SHIELDS, FY72-3

690

HIGHDENSITY BRAIO CABLE SHIELDS; FY72-4

776 (REV.)

DESIGN OF A WIRE TENSIONING DEVICE

844 (REV.)

WOVEN-WIRE TAPE VENDOR DEVELOPMENT FYT3-3

914 (REV.)

WIRE WOVEN TAPE MEASUREMENTS

FATIGUE FAILURE IN BRAIDED ELECTROMAGNETIC-RADIATION

1180

1192 (REV.)

UL TRASONIC SPOT MELD EVALUATION

14

NONOESTRUCTIVE TESTING OF PERCUSSIVE ARC MELDS

107

JOINING HIGH-STRENGTH STEELS BY DIFFUSION BONDING ' 113

PERCUSSIVE ARC WELOING FOR ELECTRICAL ASSEMBLIES 126

DEVELOPMENT OF INSTRUMENTATION FOR RELIABLE. PERCUSSIVE ARC 129 WELOING SYSTEMS

320

ALUMINUM PULSE-ARC WELDING

321

ALUMINUM PULSE-ARC WELDING

395

PERCUSSIVE-ARC VERSUS PULSE-ARC MELOING

427 


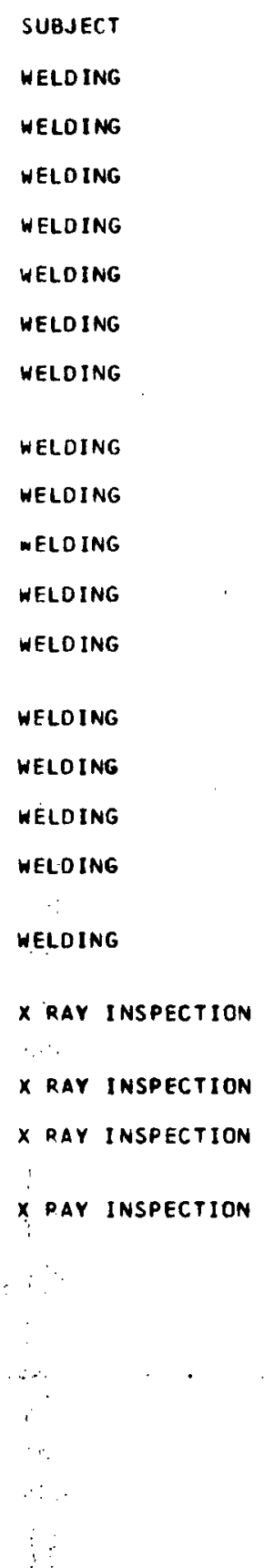

ELECTRICAL TERMINATIONS: MELDED, BRAZED, ANO BONDED, FYTL-4

472

ELECTRICAL TERMINATIONS MELDED, BRAZED AND BONOED, FYT2-1

544

ELECTRICAL TERMINATIONS: MELDED, ERAZED ANO BONDED, FYT2-2

613 (REV.) BENOIX KANSAS CITY ELECTRICAL TERMINATION WELOING HANDBOOK

637

ELECTRICAL TERMINATIONS: WELDED, BRAZED, AND BONDED, FYT2-3

662

ELECTRICAL TERMINATIONS: MELDED, BRAZED, AND BONDED, FYT2-4

773

ULTRASONIC WEL DING OF NYLON CELL CASES ON A NICKEL CADMIUM BATTERY

ULTRASONIC MELDING OF ELECTRICAL TERMINATIONS

888IREV.)

ULTRASONIC MELDING OF ELECTRICAL TERMINATIONS

1016

WELCING OF HER METIC CONNECTORS

1158(REV.)

UL TRASONICALLY WELOING ELECTRIC TERMINALS

1293 (REV.)

OESIGN MOOIFICATION FOR HIGH VOLTAGE ELECTRON BEAM WELOING

1376 (REV.) MACHINE

1488

CLOSED CIRCUIT TELEVISION WELOING ALIGNMENT SYSTEH

1533

WELDING ELECTRIC TERMINALS ULTRASONICALLY 1565

LASER WELOING OF ELECTRICAL CONNECTIONS

2022

IMPROVED PROCESS FOR WELDING STUOS TO RING ANO STUD 2031

ASSEMBLIES

2031

FINITE ELEMENT ANALYSIS OF LASER WELDING INDUCED THERMaL 2118 SHOCK

A THEORETICAL TREATMENT OF LOW VOLTAGE CONTINUOUS SPECTRUM X-RADIATION

USES OF LOW VOLTAGE X-RAY TUBES WITH THIN BERYLLIUM WINOOWS 
Appendix $G$

DRAWING SETS RELEASED 


\section{Appendix G}

DRAWING SETS RELEASED

1. Total Area Loading Compression Test Fixture

2. Air-Bearing Compression Test Fixture

3. Helical Tape Winder

4. Mandrel Rotator

5. Laser Verge and Shaft Balancing System

6. Mixing Head for Urethane Foam Metering and Mixing Machines

7. Omni-Directional Light Source

8. Rolamite Sensors

9. Miniature Gear Checking Machine

10. High Speed Scaling Counter for Laser Interferometer

11. Pantograph Positioning System for Inspecting Microcircuits

12. Percussive Arc Welding Power Supply and In-Process Monitor 
R. Bulcock, DOE-KCAO

J. H. Sund, DOE-KCAO

H. N. Meyer, Jr., DOE-ALO

S. C. Taylor, DOE-ALO

R. Caudle, DOE-MA, Washington, DC

P. W. Rosser, OR

H. R. Marron, Southfield

J. W. Weil, Southfield

R. J. Quirk/V. L. Ritter, D/010, 2A50

D. B. Badgerow, D/010, 2E 50

R. L. Calkins, D/010, 2A42

K. K. Clegg, D/010, 2E50

C. F. Kircher, D/010, 1A44

J. A. Knuth, D/010, FH31

D. J. Nigg, D/010, 2 A42

S. L. Sabanske; D/010, 2E50

J. H. Swafford, D/100, FB39

R. W. Renfrow, D/500, 1C46

J. E. Long, D/531, $1 \mathrm{~A} 46$

H. T. Barnes, D/554, BD50

L. Stratton, D/554, 2C43

G. M. Grogger, D/744, MC45

R. P. Frohmberg, D/800, 2439

D. H. $\mathrm{Hax}, \mathrm{D} / 800,2 \mathrm{~A} 41$

F. A. Spies, D/800, 2A39

A. K. Thoeni, D/800, FC39

R. E. Kessler, D/865, 2C40

I. C. Schreiber, D/865, 2E41 